\title{
Uniformly rotating smooth solutions for the incompressible 2D Euler equations
}

\author{
Angel Castro, Diego Córdoba and Javier Gómez-Serrano
}

August 9, 2018

\begin{abstract}
In this paper, we show the existence of a family of compactly supported smooth vorticities, which are solutions of the $2 \mathrm{D}$ incompressible Euler equation and rotate uniformly in time and space.
\end{abstract}

Keywords: incompressible, euler, bifurcation theory

\section{Contents}

1 Introduction .............................. 1

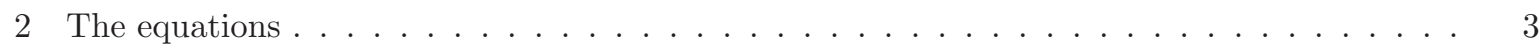

2.1 Rescaling equation $(2.6)$. . . . . . . . . . . . . . . . . . . . . . . . 5

3 Checking the hypotheses of the Crandall-Rabinowitz theorem and proof of Theorem 2.1 . . 7

3.1 Step 1. The functional Setting and the hypothesis 1 . . . . . . . . . . . . . . 7

3.2 Step 2. The derivatives . . . . . . . . . . . . . . . . . . . . . . . . . . . . . . . . . . . . . . . . . . .

3.3 Step 3. Analysis of the linear part. . . . . . . . . . . . . . . . . . . . . 17

3.3.1 One dimensionality of the Kernel of the linear operator. . . . . . . . . . . . 17

3.3.2 Codimension of the image of the linear operator. . . . . . . . . . . . . 19

3.4 Step 4. The transversality property 4. . . . . . . . . . . . . . . . . 21

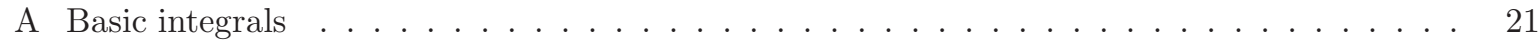

B Estimates on $A^{\prime} \ldots \ldots \ldots \ldots \ldots \ldots \ldots$

\section{Introduction}

We consider the initial value problem for the two dimensional incompressible Euler equation in vorticity form:

$$
\begin{aligned}
\partial_{t} \omega(x, t)+v(x, t) \cdot \nabla \omega(x, t) & =0, \quad(x, t) \in \mathbb{R}^{2} \times \mathbb{R}_{+} \\
v(x, t) & =\left(\nabla^{\perp}(-\Delta)^{-1} \omega\right)(x, t) \\
\omega(x, 0) & =\omega_{0}(x) .
\end{aligned}
$$

The aim of this paper is to construct a family of solutions which are compactly supported and smooth, and moreover its dynamics are given by a uniform rotation, both in time and space.

The problem of finding smooth stationary solutions has been addressed by Nadirashvili in [25], where he studies the geometry and the stability of solutions, following the works of Arnold [1, 2, 3]. 
Choffrut and Šverák 11] showed that locally near each stationary smooth solution there exists a manifold of stationary smooth solutions transversal to the foliation, and Choffrut and Székelyhidi [12] showed that there is an abundant set of stationary weak $\left(L^{\infty}\right)$ solutions near a smooth stationary one. Shvydkoy and Luo [22, 23] provided a classification of stationary smooth solutions of the form $v=\nabla^{\perp}\left(r^{\gamma} f(\theta)\right)$, where $(r, \theta)$ are polar coordinates.

Bedrossian and Masmoudi proved in 4 that given an initial perturbation of the Couette flow small in a suitable regularity class, the velocity converges strongly in $L^{2}$ to a shear flow which is also close to the Couette flow. Recently, motivated by the numerical simulations of Luo and Hou [21, Kiselev and Šverak showed in 20] double exponential growth of the gradient of the vorticity in the presence of a boundary.

It is well known that radial functions are stationary solutions for (1.1) due to the structure of the nonlinear term. The solutions that will be constructed in this paper are a smooth, compactly supported desingularization of a vortex patch, perturbed in a suitable direction. Moreover, the solutions will enjoy $m$-fold symmetry (invariance by a rotation of a $\frac{2 \pi}{m}$ angle) for $m \geq 2$.

In addition the dynamics of these solutions consist of global rotating level sets with constant angular velocity. These level sets are a perturbation of the circle. We remark that we can extend our results to the generalized Surface Quasigeostrophic (gSQG) equations in the case $0<\alpha<1$. For those values of the parameter $\alpha$, the question of global existence vs finite time singularities is still open in the smooth case and this would be to our knowledge, the first nontrivial family of solutions for which there is global existence.

These equations interpolate between the 2D incompressible Euler and the Surface Quasigeostrophic (SQG) equations and are indexed by a parameter $\alpha \in[0,2)$, where $\alpha=0$ corresponds to Euler and $\alpha=1$ to SQG. They read as follows:

$$
\left\{\begin{array}{l}
\partial_{t} \theta+u \cdot \nabla \theta=0, \quad(t, x) \in \mathbb{R}_{+} \times \mathbb{R}^{2}, \\
v=-\nabla^{\perp}(-\Delta)^{-1+\frac{\alpha}{2}} \theta, \\
\theta_{\mid t=0}=\theta_{0},
\end{array}\right.
$$

Our construction employs bifurcation theory from a patch supported in a disk which solves weakly (1.1). Our motivation to study the current problem comes from the aforementioned patch setting, where the vorticity is a characteristic function of a time-dependent set $\Omega(t)$. This property is conserved in time. Local existence in this class of solutions (assuming $C^{1, \gamma}$ regularity of the boundary) was first shown by Chemin [10 and then simplified by Bertozzi-Constantin [5 (see also 26]). A classical theorem of Yudovich [27] asserts that if the initial data is in $L^{1} \cap L^{\infty}$, then there is uniqueness within that class. In a very recent preprint, [16, the $L^{1}$ assumption can be dropped upon having an appropriate symmetry ( $m$-fold) condition.

The existence of uniformly rotating $m$-fold patches (also known as V-states) started with the numerical work of Deem and Zabusky [15. Burbea in [6] proved the existence of V-states and $C^{\infty}$ regularity for its boundary was proved by Hmidi at al. in [19]. It was shown in [9] that the boundary of these solutions is actually analytic. The doubly connected setting was studied in [14. The existence of $\mathrm{V}$-states was established for the generalized surface quasi-geostrophic equations in the range $0<\alpha<1$ in [17] and in $1 \leq \alpha<2$ in [7. We note that a desingularization from a Dirac distribution into a patch allows Hmidi and Mateu to prove the existence of corotating and counter-rotating vortex pairs in [18. In fact, our strategy is inspired by their paper.

In [8, we constructed for the case $\alpha=1$ a family of 3-fold solutions that rotated uniformly by looking at perturbations from a smooth annular profile (as opposed to a desingularization of a patch). In that case, the velocity is more singular than in 2D Euler. We had to overcome the following difficulties:

- The study of the linear problem was a functional equation, as opposed to a scalar equation 
(which was in the patch case). Even the existence of nontrivial elements in the kernel of the linear part was not evident a priori.

- There was no algebraic formula for neither the eigenvalue nor the eigenvector, not even in an implicit way (such as in [9]). This made the proof of the dimensionality of the kernel much harder since we had to show that the eigenvalue was simple and have some control of the rest of the eigenvalues. In order to do that, we resorted to a computer-assisted proof to rigorously bound the operators arising in the calculations.

The paper is organized as follows: section 2 is devoted to the reformulation of the equations (1.1) in new variables and the statement of the main theorem. In section 3 we check that our equation satisfies the hypotheses of the Crandall-Rabinowitz theorem. Finally, in Appendix A we compute some basic integrals used in the calculation of the linear part and Appendix $\mathrm{B}$ is devoted to prove some estimates used along section 3 . We note that since $\alpha<1$ and the velocity is less singular than for $\alpha=1$, we have been able to remove the computer from the estimates, as opposed to 8 .

The proofs of the theorems rely on the Crandall-Rabinowitz theorem. We recall here the statement of this theorem from [13] for expository purposes.

Theorem 1.1 (Crandall-Rabinowitz) Let $X, Y$ be Banach spaces, $V$ a neighborhood of 0 in $X$ and

$$
\begin{aligned}
\mathcal{F}: V \times(-1,1) & \rightarrow Y \\
(r, \mu) & \rightarrow \mathcal{F}[r, \mu]
\end{aligned}
$$

have the properties

1. $\mathcal{F}[0, \mu]=0$ for any $|\mu|<1$.

2. The partial derivatives $\partial_{\mu} \mathcal{F}, \partial_{r} \mathcal{F}$ and $\partial_{\mu r}^{2} \mathcal{F}$ exist and are continuous.

3. $\mathcal{N}\left(\partial_{r} \mathcal{F}[0,0]\right)$ and $Y / \mathcal{R}\left(\partial_{r} \mathcal{F}[0,0]\right)$ are one-dimensional.

4. $\partial_{\mu r}^{2} \mathcal{F}[0,0] r_{0} \notin \mathcal{R}\left(\partial_{r} \mathcal{F}[0,0]\right)$, where $\mathcal{N}\left(\partial_{r} \mathcal{F}[0,0]\right)=$ span $r_{0}$.

(Here $\mathcal{N}$ and $\mathcal{R}$ denote the kernel and range respectively). If $Z$ is any complement of $\mathcal{N}\left(\partial_{r} \mathcal{F}(0,0)\right)$ in $X$, then there is a neighborhood $U$ of $(0,0)$ in $\mathbb{R} \times X$, an interval $(-b, b)$, and continuous functions

$$
\phi:(-b, b) \rightarrow \mathbb{R} \quad \psi:(-b, b) \rightarrow Z
$$

such that $\phi(0)=0, \psi(0)=0$ and

$$
\mathcal{F}^{-1}(0) \cap U=\left\{\xi r_{0}+\xi \psi(\xi),(\phi(\xi)):|\xi|<b\right\} \cup\{(t, 0):(t, 0) \in U\} .
$$

\section{The equations}

In this section we will obtain the equation for the level sets of the vorticity of a global rotating solution of the 2D Euler equation. We assume that at time $t=0$ these level sets can be parameterized by

$$
x(\alpha, \rho)=r(\alpha, \rho)(\cos (\alpha), \sin (\alpha))
$$

where $r(\alpha, \rho)$ is a scalar function $\alpha \in \mathbb{T}$ and $\rho \in \mathbb{R}^{+}$. Since the level sets rotate with constant angular velocity the level sets of the solutions, $z(\alpha, \rho, t)$ satisfies

$$
\omega(z(\alpha, \rho, t), t)=f(\rho)
$$


for some scalar function $f$ (which does not depend neither on $\alpha$ nor on $t$ ), and can be written as follows,

$$
z(\alpha, \rho, t)=\mathcal{O}(t) x(\alpha, \rho), \quad \mathcal{O}(t)=\left(\begin{array}{cc}
\cos (\lambda t) & \sin (\lambda t) \\
-\sin (\lambda t) & \cos (\lambda t)
\end{array}\right)
$$

By using the Euler equation and (2.2) one can obtain that (see [8] for details)

$$
\left(-v(z(\alpha, \rho, t), t)+z_{t}(\alpha, \rho, t)\right) \cdot z_{\alpha}^{\perp}(\alpha, \rho, t) \frac{f_{\rho}(\rho)}{z_{\alpha}^{\perp} \cdot z_{\rho}(\alpha, \rho, t)}=0
$$

where

$$
v(x, t)=\frac{1}{2 \pi} \int_{\mathbb{R}^{2}} \log (|x-y|) \nabla^{\perp} \omega(y, t) d y,
$$

and by a change of coordinates we have that

$$
v(z(\alpha, \rho, t), t)=\frac{1}{2 \pi} \int_{0}^{\infty} \int_{-\pi}^{\pi} \log \left(\left|z(\alpha, \rho, t)-z\left(\alpha^{\prime}, \rho^{\prime}, t\right)\right|\right) f_{\rho}\left(\rho^{\prime}\right) z_{\alpha}\left(\alpha^{\prime}, \rho^{\prime}, t\right) d \alpha^{\prime} d \rho^{\prime} .
$$

Thus equation (2.4) can be written in terms of $x(\alpha, \rho)$ in the following way

$$
\lambda x(\alpha, \rho) \cdot x_{\alpha}(\alpha, \rho)+\frac{1}{2 \pi} x_{\alpha}^{\perp}(\alpha, \rho) \cdot \int_{0}^{\infty} \int_{-\pi}^{\pi} f_{\rho}\left(\rho^{\prime}\right) \log \left(\left|x(\alpha, \rho)-x\left(\alpha^{\prime}, \rho^{\prime}\right)\right|\right) x_{\alpha}\left(\alpha^{\prime}, \rho^{\prime}\right) d \alpha^{\prime} d \rho^{\prime}=0 .
$$

for $\alpha \in \mathbb{T}$ and $\rho \in \operatorname{supp}\left(f_{\rho}\right)$.

In terms of the function $r(\alpha, \rho)$ we get

$$
\begin{aligned}
& \mathcal{F}_{f}[r, \lambda] \\
& \equiv \lambda r(\alpha, \rho) r_{\alpha}(\alpha, \rho)+\frac{r(\alpha, \rho)}{2 \pi} \int_{0}^{\infty} \int_{-\pi}^{\pi} f_{\rho}\left(\rho^{\prime}\right) \log \left(\left|x(\alpha, \rho)-x\left(\alpha^{\prime}, \rho^{\prime}\right)\right|\right) \cos \left(\alpha-\alpha^{\prime}\right) r_{\alpha}\left(\alpha^{\prime}, \rho^{\prime}\right) d \alpha^{\prime} d \rho^{\prime} \\
& -\frac{r_{\alpha}(\alpha, \rho)}{2 \pi} \int_{0}^{\infty} \int_{-\pi}^{\pi} f_{\rho}\left(\rho^{\prime}\right) \log \left(\left|x(\alpha, \rho)-x\left(\alpha^{\prime}, \rho^{\prime}\right)\right|\right) \cos \left(\alpha-\alpha^{\prime}\right) r\left(\alpha^{\prime}, \rho^{\prime}\right) d \alpha^{\prime} d \rho^{\prime} \\
& +\frac{1}{2 \pi} \int_{0}^{\infty} \int_{-\pi}^{\pi} f_{\rho}\left(\rho^{\prime}\right) \log \left(\left|x(\alpha, \rho)-x\left(\alpha^{\prime}, \rho^{\prime}\right)\right|\right) \sin \left(\alpha-\alpha^{\prime}\right)\left(r(\alpha, \rho) r\left(\alpha^{\prime}, \rho^{\prime}\right)+r_{\alpha}(\alpha, \rho) r_{\alpha}\left(\alpha^{\prime}, \rho^{\prime}\right)\right) d \alpha^{\prime} d \rho^{\prime} \\
& =0
\end{aligned}
$$

for $\alpha \in \mathbb{T}$ and $\rho \in(1-a, 1+a)$ and $\left|x(\alpha, \rho)-x\left(\alpha^{\prime}, \rho^{\prime}\right)\right|=\sqrt{r(\alpha, \rho)^{2}+r\left(\alpha^{\prime}, \rho^{\prime}\right)^{2}-2 r(\alpha, \rho) r\left(\alpha^{\prime}, \rho^{\prime}\right) \cos \left(\alpha-\alpha^{\prime}\right)}$.

We discuss the kind of $f$ we will use in this paper.

First we define the profile $F(\rho)$

$$
F(\rho)=\left\{\begin{array}{cc}
1 & \rho \in(-\infty,-1] \\
1+\int_{-1}^{\rho} \phi\left(\rho^{\prime}\right) d \rho^{\prime} & \rho \in(-1,1) \\
0 & \rho \in[1, \infty)
\end{array}\right.
$$

where $\int_{-1}^{1} \phi\left(\rho^{\prime}\right) d \rho^{\prime}=-1$ and $\phi \in C_{c}^{3}((-1,1))$. Then the function $f^{a}(\rho)$ (we make explicit the dependence in the parameter $a$ ) will be given by

$$
f^{a}(\rho)=F\left(\frac{\rho-1}{a}\right)
$$


and it is easy to check that $\operatorname{supp}\left(f_{\rho}^{a}\right) \subset(1-a, 1+a)$. We also remark that $f_{\rho}^{a}(1+a s)$ is independent of $a$, a fact that will be used later.

The parameter $a$ will be used as the bifurcation parameter in order to find solutions for (2.6). Indeed the angular velocity $\lambda$ will depend on $a$ (we will make explicit this dependence later). Thus, we will solve the equation

$$
\mathcal{F}[r, a]=0
$$

with $\mathcal{F}[r, a]=\mathcal{F}_{f^{a}}[r, \lambda(a)]$.

\subsection{Rescaling equation (2.6)}

In order to find solutions we start by rescaling the equation (2.6). Changing variables, $s=\frac{\rho-1}{a}$, one obtains that

$$
\begin{aligned}
& \lambda(a) r(\alpha, 1+a s) r_{\alpha}(\alpha, 1+a s) \\
& +\frac{r(\alpha, 1+a s)}{2 \pi} \int_{-1}^{1} \int_{-\pi}^{\pi} F_{\rho}\left(s^{\prime}\right) \log \left(\left|x(\alpha, 1+a s)-x\left(\alpha^{\prime}, 1+a s^{\prime}\right)\right|\right) \cos \left(\alpha-\alpha^{\prime}\right) r_{\alpha}\left(\alpha^{\prime}, 1+a s^{\prime}\right) d \alpha^{\prime} d s^{\prime} \\
& -\frac{r_{\alpha}\left(\alpha, 1+a s^{\prime}\right)}{2 \pi} \int_{-1}^{1} \int_{-\pi}^{\pi} F_{\rho}\left(s^{\prime}\right) \log \left(\left|x(\alpha, 1+a s)-x\left(\alpha^{\prime}, 1+a s^{\prime}\right)\right|\right) \cos \left(\alpha-\alpha^{\prime}\right) r\left(\alpha^{\prime}, 1+a s^{\prime}\right) d \alpha^{\prime} d s^{\prime} \\
& +\frac{1}{2 \pi} \int_{-1}^{1} \int_{-\pi}^{\pi} F_{\rho}\left(s^{\prime}\right) \log \left(\left|x(\alpha, 1+a s)-x\left(\alpha^{\prime}, 1+a s^{\prime}\right)\right|\right) \sin \left(\alpha-\alpha^{\prime}\right) r(\alpha, 1+a s) r\left(\alpha^{\prime}, 1+a s^{\prime}\right) d \alpha^{\prime} d s^{\prime}, \\
& \left.+\frac{1}{2 \pi} \int_{-1}^{1} \int_{-\pi}^{\pi} F_{\rho}\left(s^{\prime}\right) \log \left(\left|x(\alpha, 1+a s)-x\left(\alpha^{\prime}, 1+a s^{\prime}\right)\right|\right) \sin \left(\alpha-\alpha^{\prime}\right) r_{\alpha}(\alpha, 1+a s) r_{\alpha}\left(\alpha^{\prime}, 1+a s^{\prime}\right)\right) d \alpha^{\prime} d s^{\prime}, \\
& =0,
\end{aligned}
$$

for $\alpha \in(-\pi, \pi]$ and $s \in(-1,1)$.

The equation we will try to solve is the one we obtain dividing (2.8) by $a^{2}$ and by replacing $r(\alpha, 1+$ as) by $1+a^{2}(s+\tilde{r}(\alpha, s))$ (we will solve in $a$ and $\tilde{r}$ ), in such a way that $r(\alpha, \rho)=1-a+a \rho+a^{2} \tilde{r}\left(\alpha, \frac{\rho-1}{a}\right)$ will be a solution of (2.7) for any $a \neq 0$. This equation reads

$$
\begin{aligned}
& \lambda(a)\left(1+a^{2}(s+\tilde{r}(\alpha, s))\right) \tilde{r}_{\alpha}(\alpha, s) \\
& +\frac{1+a^{2} \tilde{r}(\alpha, s)}{4 \pi} \int_{-1}^{1} \int_{-\pi}^{\pi} F_{\rho}\left(s^{\prime}\right) \log (A[\tilde{r}, a]) \cos \left(\alpha-\alpha^{\prime}\right) \tilde{r}_{\alpha}\left(\alpha^{\prime}, s^{\prime}\right) d \alpha^{\prime} d s^{\prime} \\
& -\frac{\tilde{r}_{\alpha}(\alpha, s)}{4 \pi} \int_{-1}^{1} \int_{-\pi}^{\pi} F_{\rho}\left(s^{\prime}\right) \log (A[\tilde{r}, a]) \cos \left(\alpha-\alpha^{\prime}\right)\left(1+a^{2}\left(s^{\prime}+\tilde{r}\left(\alpha^{\prime}, s^{\prime}\right)\right)\right) d \alpha^{\prime} d s^{\prime} \\
& +\frac{1}{4 \pi} \int_{-1}^{1} \int_{-\pi}^{\pi} F_{\rho}\left(s^{\prime}\right) \frac{1}{a^{2}} \log (A[\tilde{r}, a]) \sin \left(\alpha-\alpha^{\prime}\right) d \alpha^{\prime} d s^{\prime}, \\
& +\frac{1}{4 \pi} \int_{-1}^{1} \int_{-\pi}^{\pi} F_{\rho}\left(s^{\prime}\right) \log (A[\tilde{r}, a]) \sin \left(\alpha-\alpha^{\prime}\right)\left(s+\tilde{r}(\alpha, s)+s^{\prime}+\tilde{r}\left(\alpha^{\prime}, s^{\prime}\right)\right) d \alpha^{\prime} d s^{\prime}, \\
& +\frac{a^{2}}{4 \pi} \int_{-1}^{1} \int_{-\pi}^{\pi} F_{\rho}\left(s^{\prime}\right) \log (A[\tilde{r}, a]) \sin \left(\alpha-\alpha^{\prime}\right)\left(\tilde{r}_{\alpha}(\alpha, s) \tilde{r}_{\alpha}\left(\alpha^{\prime}, s^{\prime}\right)+(s+\tilde{r}(\alpha, s))\left(s^{\prime}+\tilde{r}\left(\alpha^{\prime}, s^{\prime}\right)\right)\right) d \alpha^{\prime} d s^{\prime}=0,
\end{aligned}
$$

with 


$$
\begin{aligned}
& A[\tilde{r}, a]=A[\tilde{r}, a]\left(s, s^{\prime}, \alpha, \alpha^{\prime}\right) \\
& =\left(1+a^{2}(s+\tilde{r}(\alpha, s))\right)^{2}+\left(1+a^{2}\left(s^{\prime}+\tilde{r}\left(\alpha^{\prime}, s^{\prime}\right)\right)\right)^{2}-2\left(1+a^{2}(s+\tilde{r}(\alpha, s))\right)\left(1+a^{2}\left(s^{\prime}+\tilde{r}\left(\alpha^{\prime}, s^{\prime}\right)\right)\right) \cos \left(\alpha-\alpha^{\prime}\right) \\
& =4 \sin ^{2}\left(\frac{\alpha-\alpha^{\prime}}{2}\right)+4 a^{2}\left(s+\tilde{r}(\alpha, s)+s^{\prime}+\tilde{r}\left(\alpha^{\prime}, s^{\prime}\right)\right) \sin ^{2}\left(\frac{\alpha-\alpha^{\prime}}{2}\right) \\
& +a^{4}\left(4(s+\tilde{r}(\alpha, s))\left(s^{\prime}+\tilde{r}\left(\alpha^{\prime}, s^{\prime}\right)\right) \sin ^{2}\left(\frac{\alpha-\alpha^{\prime}}{2}\right)+\left(s+\tilde{r}(\alpha, s)-s-\tilde{r}\left(\alpha^{\prime}, s^{\prime}\right)\right)^{2}\right) .
\end{aligned}
$$

Since $A[\tilde{r}, 0]=4 \sin ^{2}\left(\frac{\alpha-\alpha^{\prime}}{2}\right)$ we have that

$$
\begin{aligned}
& \frac{1}{4 \pi} \int_{-1}^{1} \int_{-\pi}^{\pi} F_{\rho}\left(s^{\prime}\right) \frac{1}{a} \log (A[\tilde{r}, a]) \sin \left(\alpha-\alpha^{\prime}\right) d \alpha^{\prime} d s^{\prime} \\
& =\frac{1}{4 \pi} \int_{-1}^{1} \int_{-\pi}^{\pi} F_{\rho}\left(s^{\prime}\right) \frac{1}{a} \log \left(\frac{A[\tilde{r}, a]}{A[\tilde{r}, 0]}\right) \sin \left(\alpha-\alpha^{\prime}\right) d \alpha^{\prime} d s^{\prime}
\end{aligned}
$$

Therefore we need to solve the equation

$$
\mathcal{G}[\tilde{r}, a]=0
$$

with

$$
\begin{aligned}
& \mathcal{G}[\tilde{r}, a] \\
& =\lambda(a)\left(1+a^{2}(s+\tilde{r}(\alpha, s))\right) \tilde{r}_{\alpha}(\alpha, s) \\
& +\frac{1+a^{2}(s+\tilde{r}(\alpha, s))}{4 \pi} \int_{-1}^{1} \int_{-\pi}^{\pi} F_{\rho}\left(s^{\prime}\right) \log (A[\tilde{r}, a]) \cos \left(\alpha-\alpha^{\prime}\right) \tilde{r}_{\alpha}\left(\alpha^{\prime}, s^{\prime}\right) d \alpha^{\prime} d s^{\prime} \\
& -\frac{\tilde{r}_{\alpha}(\alpha, s)}{4 \pi} \int_{-1}^{1} \int_{-\pi}^{\pi} F_{\rho}\left(s^{\prime}\right) \log (A[\tilde{r}, a]) \cos \left(\alpha-\alpha^{\prime}\right)\left(1+a^{2}\left(s^{\prime}+\tilde{r}\left(\alpha^{\prime}, s^{\prime}\right)\right)\right) d \alpha^{\prime} d s^{\prime} \\
& +\frac{1}{4 \pi} \int_{-1}^{1} \int_{-\pi}^{\pi} F_{\rho}\left(s^{\prime}\right) \frac{1}{a^{2}} \log \left(\frac{A[\tilde{r}, a]}{A[\tilde{r}, 0]}\right) \sin \left(\alpha-\alpha^{\prime}\right) d \alpha^{\prime} d s^{\prime}, \\
& +\frac{1}{4 \pi} \int_{-1}^{1} \int_{-\pi}^{\pi} F_{\rho}\left(s^{\prime}\right) \log (A[\tilde{r}, a]) \sin \left(\alpha-\alpha^{\prime}\right)\left(s+\tilde{r}(\alpha, s)+s^{\prime}+\tilde{r}\left(\alpha^{\prime}, s^{\prime}\right) d \alpha^{\prime} d s^{\prime},\right. \\
& +\frac{a^{2}}{4 \pi} \int_{-1}^{1} \int_{-\pi}^{\pi} F_{\rho}\left(s^{\prime}\right) \log (A[\tilde{r}, a]) \sin \left(\alpha-\alpha^{\prime}\right)\left(\tilde{r}_{\alpha}(\alpha, s) \tilde{r}_{\alpha}\left(\alpha^{\prime}, s^{\prime}\right)+(s+\tilde{r}(\alpha, s))\left(s^{\prime}+\tilde{r}\left(\alpha^{\prime}, s^{\prime}\right)\right)\right) d \alpha^{\prime} d s^{\prime},
\end{aligned}
$$

We now state the main theorem of this paper:

Theorem 2.1 Consider the domain

$$
\Omega \equiv\{(\alpha, s): \alpha \in \mathbb{T},-1<s<1\} .
$$

and let the function $\phi$ be as in section 圆, Let $m \geq 2$ be an integer and $\lambda(a)=\lambda_{m}(0)+\frac{d \lambda}{d a}(0) a$, with $\lambda_{m}(0)=\frac{m-1}{2 m}$ and $\frac{d \lambda}{d a}(0) \neq 0$. Then there exists a branch of nontrivial smooth solutions, with $m-$ fold symmetry, of equation (2.9), in $H^{4,3}(\Omega)$, bifurcating from $\tilde{r}(\alpha, s)=0$ and $a=0$.

The proof of Theorem 2.1 consists of checking the hypotheses of Theorem 1.1. This is done in the next section. 
Corollary 2.2 There exist global rotating solutions for the 2D-Euler vorticity equation with $C^{2}$ regularity with compact support, with $m$-fold symmetry for any integer $m \geq 2$.

Proof: We fix $a>0$ small enough in such a way that $\tilde{r}(\alpha, \rho)$ is the solution of (2.9), given by Theorem 2.1 for that $a$. Then $r(\alpha, \rho)=1-a+a \rho+a^{2} \tilde{r}\left(\alpha, \frac{\rho-1}{a}\right)$ is a solution of equation (2.7). In order to check that the vorticity defined through the equations (2.1), (2.2) and (2.3) is a $C^{2}$ rotating solution of the 2D Euler equation we just need to check that $\partial_{\rho} r(\alpha, \rho)>0$ (in order to have that $z(\alpha, \rho)$ is one-to-one in $\alpha \in \mathbb{T}, \rho \in[1-a, 1+a]$. But $\partial_{\rho} r(\alpha, \rho)=a\left(1+\left(\partial_{s} \tilde{r}\right)\left(\alpha, \frac{\rho-1}{a}\right)\right)$ and since we are bifurcating from $\tilde{r}=0$ the result holds for small enough $a$.

Remark 2.3 We notice that that the sufficient stability condition [24, equation (3.10), p. 116] may not be satisfied by our solutions (take for example any positive $f^{a}(\rho)$ ).

\section{Checking the hypotheses of the Crandall-Rabinowitz theo- rem and proof of Theorem 2.1}

\subsection{Step 1. The functional Setting and the hypothesis 1}

The beginning of this section is devoted to defining the spaces we will work with. After that the main purpose will be to prove lemma 3.1 .

Let us also define the spaces $H^{4,3}(\Omega)$ as follows:

$$
r \in H^{4,3} \Leftrightarrow\left\{r \in L^{2}(\Omega):\|r\|_{L^{2}(\Omega)}^{2}+\left\|\partial_{\rho}^{3} r\right\|_{L^{2}(\Omega)}^{2}+\sum_{j=0}^{3}\left\|\partial_{\alpha}^{4-j} \partial_{\rho}^{j} r\right\|_{L^{2}(\Omega)}^{2}<\infty\right\} .
$$

Moreover, we will say that $r \in H_{m, \text { even }}^{4,3}$ (resp. $r \in H_{m, o d d}^{4,3}$ ) if $r \in H^{4,3}$, has $m$-fold symmetry and is even (resp. odd).

Lemma 3.1 Let $\mathcal{G}$ be as in (2.10). Then there exist numbers $\delta>0$ and $a_{0}>0$ small enough such that

$$
\begin{aligned}
\mathcal{G}: V^{\delta} \times\left(-a_{0}, a_{0}\right) & \rightarrow H_{m, o d d}^{3,3}(\Omega), \\
(r, a) & \rightarrow \mathcal{G}[r, a] .
\end{aligned}
$$

where

$$
V^{\delta}=\left\{r \in H^{4,3}(\Omega):\|r\|_{H_{m, \text { even }}^{4,3}(\Omega)}<\delta\right\}
$$

Proof:

We will use the following notation. Given any function $f(\alpha, s)$ we will write

$$
\begin{aligned}
f & =f(\alpha, s) \\
f^{\prime} & =f\left(\alpha-\alpha^{\prime}, s-s^{\prime}\right) .
\end{aligned}
$$

In addition either we will omit the dependence of the $A[\tilde{r}, a]\left(s, s^{\prime}, \alpha, \alpha^{\prime}\right)$ on $\tilde{r}, a, s, s^{\prime}, \alpha$ and $\alpha^{\prime}$ or we will only make it explicit with respect to the parameters we are interested in. Also

$$
A^{\prime} \equiv A[\tilde{r}, a]\left(s, s-s^{\prime}, \alpha-\alpha^{\prime}\right) .
$$

We will use the following convention to take derivatives: $\partial^{k}$ means either $\partial_{\alpha}^{k}$ or $\partial_{\rho}^{k}$. 
It will be convenient to alleviate the notation to use the function $u(\alpha, s)=s+\tilde{r}(\alpha, s)$ in such a way that

$$
\begin{aligned}
& \mathcal{G}[u-s, a] \\
& =\lambda(a)\left(1+a^{2} u\right) u_{\alpha} \\
& +\frac{1+a^{2} u}{4 \pi} \int_{-\infty}^{\infty} \int_{-\pi}^{\pi} F_{\rho}\left(s-s^{\prime}\right) \log \left(A^{\prime}\right) \cos \left(\alpha^{\prime}\right) u_{\alpha}^{\prime} d \alpha^{\prime} d s^{\prime} \\
& \left.-\frac{u_{\alpha}}{4 \pi} \int_{-\infty}^{\infty} \int_{-\pi}^{\pi} F_{\rho}\left(s-s^{\prime}\right) \log \left(A^{\prime}\right) \cos \left(\alpha^{\prime}\right)\left(1+a^{2} u^{\prime}\right)\right) d \alpha^{\prime} d s^{\prime} \\
& +\frac{1}{4 \pi} \int_{-\infty}^{\infty} \int_{-\pi}^{\pi} F_{\rho}\left(s-s^{\prime}\right) \frac{1}{a^{2}} \log \left(\frac{A^{\prime}}{A^{\prime}[a=0]}\right) \sin \left(\alpha^{\prime}\right) d \alpha^{\prime} d s^{\prime}, \\
& +\frac{1}{4 \pi} \int_{-\infty}^{\infty} \int_{-\pi}^{\pi} F_{\rho}\left(s-s^{\prime}\right) \log \left(A^{\prime}\right) \sin \left(\alpha^{\prime}\right)\left(u+u^{\prime}\right) d \alpha^{\prime} d s^{\prime}, \\
& +\frac{a^{2}}{4 \pi} \int_{-\infty}^{\infty} \int_{-\pi}^{\pi} F_{\rho}\left(s-s^{\prime}\right) \log \left(A^{\prime}\right) \sin \left(\alpha^{\prime}\right)\left(u_{\alpha} u_{\alpha}^{\prime}+u u^{\prime}\right) d \alpha^{\prime} d s^{\prime}, \\
& \equiv \sum_{i=0}^{5} \mathcal{G}_{i}[\tilde{r}, a] .
\end{aligned}
$$

The proof of the estimates on $A$ that will be used to prove the lemma are left to the Appendix $\mathrm{B}$

We will bound the terms $\mathcal{G}_{i}[\tilde{r}, a]$ in the equation (2.10) in $H^{3}(\Omega)$. The bound for $\mathcal{G}_{0}[\tilde{r}, a]$ is straightforward since $H^{4,3} \subset C^{2}$, as it is proven in [8, Lemma 4.1].

The proof for $\mathcal{G}_{i}[\tilde{r}, a]$, with $i=1,2,4,5$ is similar. We will give the details only for $\mathcal{G}_{1}[\tilde{r}, a]$. Finally we will bound $\mathcal{G}_{3}[\tilde{r}, a]$.

The $L^{2}-$ norm of $\mathcal{G}_{1}[u-s, a]$ it is easy to bound by using Lemma B.1-2, then, in order to bound $\mathcal{G}_{3}[u-s, a]$ in $H^{3}$, we take three derivatives to obtain

$$
\begin{aligned}
& \partial^{3} \mathcal{G}_{1}[u-s, a]=\frac{1}{4 \pi} \int_{-\infty}^{\infty} \int_{-\pi}^{\pi} \cos \left(\alpha^{\prime}\right)\left(1+a^{2} u\right) F_{\rho}\left(s-s^{\prime}\right) u_{\alpha}^{\prime} \partial^{3} \log \left(A^{\prime}\right) d \alpha^{\prime} d s^{\prime} \\
& +\frac{1}{4 \pi} \int_{-\infty}^{\infty} \int_{-\pi}^{\pi}\left(\partial^{3}\left(\log \left(A^{\prime}\right) \cos \left(\alpha^{\prime}\right)\left(1+a^{2} u\right) F_{\rho}\left(s-s^{\prime}\right) u_{\alpha}^{\prime}\right)-\left(1+a^{2} u\right) F_{\rho}\left(s-s^{\prime}\right) u_{\alpha}^{\prime} \partial^{3} \log \left(A^{\prime}\right)\right) d \alpha^{\prime} d s^{\prime} .
\end{aligned}
$$

Remark 3.2 Actually we also need to take mixed derivatives, as for example $\partial_{\alpha}^{2} \partial_{\rho}$. We will not compute explicitly these mixed derivatives but similar arguments with small modifications apply to them.

To estimate the first term in the right hand side of the previous expression we use lemma B.5 and to bound the second one we use B.4

We now bound $\mathcal{G}_{3}$. The estimate of the $L^{2}$-norm follows from lemma B.8. Then we are concerned with the bound in $L^{2}$ of $\partial^{3} \mathcal{G}_{3}$,

$$
\begin{aligned}
& \partial^{3} \mathcal{G}_{3}[u-s, a]=\frac{1}{4 \pi} \int_{-\infty}^{\infty} \int_{-\pi}^{\pi} F_{\rho}\left(s-s^{\prime}\right) \sin \left(\alpha^{\prime}\right) \frac{1}{a^{2}} \partial^{3} \log \left(A^{\prime}\right) d \alpha^{\prime} d s^{\prime} \\
& +\frac{1}{4 \pi} \int_{-\infty}^{\infty} \int_{-\pi}^{\pi}\left(\partial^{3}\left(F_{\rho}\left(s-s^{\prime}\right) \frac{1}{a^{2}} \log \left(\frac{A^{\prime}}{A^{\prime}[0]}\right)\right)-F_{\rho}\left(s-s^{\prime}\right) \frac{1}{a^{2}} \partial^{3} \log \left(A^{\prime}\right)\right) \sin \left(\alpha^{\prime}\right) d \alpha^{\prime} d s^{\prime} .
\end{aligned}
$$

To estimate the fist term in the right hand side of the previous expression we can use lemma B.5 To bound the second one we can use lemmas B.8 and B.4. We remark that we could use the cancellation 
given by the factor $\sin \left(\alpha^{\prime}\right)$ in the integrand of $\mathcal{G}_{3}$ but actually we do not need it to prove the estimate of $\mathcal{G}_{3}$ in $H^{3}$.

In addition we need to prove that $\mathcal{G}[\tilde{r}, a]$ is continuous with respect to $a$ and $\tilde{r}$ in $\left(-a_{0}, a_{0}\right) \times V^{\delta}$. In the next section we will prove that, in fact, it is $C^{1}$ in this domain.

Therefore, in order to prove that,

$$
\mathcal{G}: V \times(-1,1) \rightarrow H_{3, \text { odd }}^{3,3}\left(\Omega_{a}\right)
$$

we just need to show that if

$$
\tilde{r}(-\alpha, \rho)=\tilde{r}(\alpha, \rho)
$$

and

$$
\tilde{r}\left(\alpha+\frac{2 n \pi}{m}, \rho\right)=\tilde{r}(\alpha, \rho)
$$

for $n \in \mathbb{N}$, then

$$
\mathcal{G}(-\alpha, \rho)=-\mathcal{G}(\alpha, \rho)
$$

and

$$
\mathcal{G}\left(\alpha+\frac{2 n \pi}{m}, \rho\right)=\mathcal{G}(\alpha, \rho)
$$

for $n \in \mathbb{N}$. These two properties are easy to check.

The last part of this section will be to check that the hypothesis 1 in the C-R theorem holds. This fact is a consequence of radial functions being stationary solutions of the Euler equation but let us check it on (2.10). If we take $\tilde{r}=0$, the only term in (2.10) that is not trivially zero is $\mathcal{G}_{3}[0, a]$. If $a \neq 0$, then it is immediate following from the oddness of the integrand in $\alpha^{\prime}$. If $a=0$,

$$
\lim _{a \rightarrow 0} \mathcal{G}_{3}[0, a]=\frac{1}{4 \pi} \int_{-\infty}^{\infty} \int_{-\pi}^{\pi} F_{\rho}\left(s-s^{\prime}\right) \frac{\left(\partial_{a^{2}} A^{\prime}\right)[0,0]}{A^{\prime}[0,0]} \sin \left(\alpha-\alpha^{\prime}\right) d \alpha^{\prime} d s^{\prime}
$$

where the integrand is also odd in $\alpha^{\prime}$.

\subsection{Step 2. The derivatives}

Lemma 3.3 The derivative $\partial_{a} \mathcal{G}[u-s, a]$ is given by:

$$
\begin{aligned}
\partial_{a} \mathcal{G}[u-s, a] & =\frac{d \lambda}{d a}(a)\left(1+a^{2} u\right) u_{\alpha}+2 a \lambda(a) u u_{\alpha} \\
& +\frac{a u}{2 \pi} \int_{-\infty}^{\infty} \int_{-\pi}^{\pi} F_{\rho}\left(s-s^{\prime}\right) \log \left(A^{\prime}\right) \cos \left(\alpha^{\prime}\right) u_{\alpha}^{\prime} d \alpha^{\prime} d s^{\prime} \\
& +\frac{1+a^{2} u}{4 \pi} \int_{-\infty}^{\infty} \int_{-\pi}^{\pi} F_{\rho}\left(s-s^{\prime}\right) \frac{\partial_{a} A^{\prime}}{A^{\prime}} \cos \left(\alpha^{\prime}\right) u_{\alpha}^{\prime} d \alpha^{\prime} d s^{\prime} \\
& -\frac{u_{\alpha}}{4 \pi} \int_{-\infty}^{\infty} \int_{-\pi}^{\pi} F_{\rho}\left(s-s^{\prime}\right) \frac{\partial_{a} A^{\prime}}{A^{\prime}} \cos \left(\alpha^{\prime}\right)\left(1+a^{2} u^{\prime}\right) d \alpha^{\prime} d s^{\prime}
\end{aligned}
$$




$$
\begin{aligned}
& -\frac{a u_{\alpha}}{2 \pi} \int_{-\infty}^{\infty} \int_{-\pi}^{\pi} F_{\rho}\left(s-s^{\prime}\right) \log \left(A^{\prime}\right) \cos \left(\alpha^{\prime}\right) u^{\prime} d \alpha^{\prime} d s^{\prime} \\
& -\frac{1}{4 \pi} \int_{-\infty}^{\infty} \int_{-\pi}^{\pi} F_{\rho}\left(s-s^{\prime}\right) \partial_{a}\left(\frac{1}{a^{2}} \log \left(\frac{A^{\prime}}{A^{\prime}[a=0]}\right)\right) \sin \left(\alpha^{\prime}\right) d \alpha^{\prime} d s^{\prime} \\
& +\frac{1}{4 \pi} \int_{-\infty}^{\infty} \int_{-\pi}^{\pi} F_{\rho}\left(s-s^{\prime}\right) \frac{\partial_{a} A^{\prime}}{A^{\prime}} \sin \left(\alpha^{\prime}\right)\left(u+u^{\prime}\right) d \alpha^{\prime} d s^{\prime}, \\
& +\frac{a^{2}}{4 \pi} \int_{-\infty}^{\infty} \int_{-\pi}^{\pi} F_{\rho}\left(s-s^{\prime}\right) \frac{\partial_{a} A^{\prime}}{A^{\prime}} \sin \left(\alpha^{\prime}\right)\left(u u^{\prime}+u_{\alpha} u_{\alpha}^{\prime}\right) d \alpha^{\prime} d s^{\prime}, \\
& +\frac{a}{2 \pi} \int_{-\infty}^{\infty} \int_{-\pi}^{\pi} F_{\rho}\left(s-s^{\prime}\right) \log \left(A^{\prime}\right) \sin \left(\alpha^{\prime}\right)\left(u u^{\prime}+u_{\alpha} u_{\alpha}^{\prime}\right) d \alpha^{\prime} d s^{\prime},
\end{aligned}
$$

Moreover this derivative is continuous in $H^{3}$.

Proof: We will give the details of the differentiability with respect to $a$ of the term

$$
\mathcal{G}_{11}[u-s, a]=\frac{1}{4 \pi} \int_{-\infty}^{\infty} \int_{-\pi}^{\pi} F_{s}\left(s-s^{\prime}\right) \cos \left(\alpha^{\prime}\right) \log \left(A^{\prime}\right) u_{\alpha}^{\prime} d \alpha^{\prime} d s^{\prime}
$$

and of $\mathcal{G}_{3}$ which are the most singular ones. First we notice that for $a \neq 0$ the formula

$$
\partial_{a} \mathcal{G}_{11}[u-s, a]=\frac{1}{4 \pi} \int_{-\infty}^{\infty} \int_{-\pi}^{\pi} F_{s}\left(s-s^{\prime}\right) \cos \left(\alpha^{\prime}\right) \frac{\partial_{a} A^{\prime}}{A^{\prime}} u_{\alpha}^{\prime} d \alpha^{\prime} d s^{\prime}
$$

holds. In addition, by using lemmas B.10 and B.11 we obtain that, for $a \neq 0, \partial_{a} \mathcal{G}_{11} \in H^{3}$ and that $\lim _{a \rightarrow 0^{ \pm}} \partial_{a} \mathcal{G}_{11}[u-s, a]=0$ in $H^{3}$. It remains to prove that $\lim _{h \rightarrow 0^{ \pm}} \frac{\mathcal{G}_{11}[u-s, h]-\mathcal{G}_{11}[u-s, 0]}{h}=0$ in $H^{3}$. This is a consequence of lemmas B.12 and B.13.

Next we compute the derivative with respect to $a$ of $\mathcal{G}_{3}$ at the point $a=0$. First we show that

$$
\lim _{a \rightarrow 0} \mathcal{G}_{3}[u-s, a]=\frac{1}{4 \pi} \int_{-\infty}^{\infty} \int_{-\pi}^{\pi} F_{\rho}\left(s-s^{\prime}\right)\left(u+u^{\prime}\right) \sin \left(\alpha^{\prime}\right) d \alpha^{\prime} d s^{\prime}
$$

This is done by using lemmas B.15 and B.16

Thus

$$
\mathcal{G}_{3}[u-s, a]-\mathcal{G}_{3}[u-s, 0]=\frac{1}{4 \pi} \int_{-\infty}^{\infty} \int_{-\pi}^{\pi} F_{\rho}\left(s-s^{\prime}\right)\left(\frac{1}{a^{2}} \log \left(\frac{A^{\prime}[a]}{A^{\prime}[0]}\right)-\left(u+u^{\prime}\right)\right) \sin \left(\alpha^{\prime}\right) d \alpha^{\prime} d s^{\prime}
$$

And we can check that

$$
\lim _{a \rightarrow 0} \frac{\mathcal{G}_{3}[u-s, a]-\mathcal{G}_{3}[u-s, 0]}{a}=0
$$

in $H^{3}$ by using again lemmas B.15 and B.16

In addition we have that

$$
\frac{1}{4 \pi} \int_{-\infty}^{\infty} \int_{-\pi}^{\pi} F_{\rho}\left(s-s^{\prime}\right) \partial_{a}\left(\frac{1}{a^{2}} \log \left(\frac{A^{\prime}}{A^{\prime}[a=0]}\right)\right) \sin \left(\alpha^{\prime}\right) d \alpha^{\prime} d s^{\prime}
$$

is in $H^{3}$ for every $a \neq 0$ and that

$$
\lim _{a \rightarrow 0} \frac{1}{4 \pi} \int_{-\infty}^{\infty} \int_{-\pi}^{\pi} F_{\rho}\left(s-s^{\prime}\right) \partial_{a}\left(\frac{1}{a^{2}} \log \left(\frac{A^{\prime}}{A^{\prime}[a=0]}\right)\right) \sin \left(\alpha^{\prime}\right) d \alpha^{\prime} d s^{\prime}=0
$$

in $H^{3}$ by lemma B.18 and B.19. 
Finally we will prove the continuity with respect to $u$. Let us focus on the term

$$
\partial_{a} \mathcal{G}_{11}[u-s, a]=\frac{1}{4 \pi} \int_{-\infty}^{\infty} \int_{-\pi}^{\pi} F_{s}\left(s-s^{\prime}\right) \cos \left(\alpha^{\prime}\right) \frac{\partial A^{\prime}[u]}{A^{\prime}[u]} u_{\alpha}^{\prime} d \alpha^{\prime} d s^{\prime} .
$$

We want to estimate the difference $\left\|\partial_{a} \mathcal{G}_{11}[u-s, a]-\partial_{a} \mathcal{G}_{11}[v-s, a]\right\|_{H^{3}}$, with $u, v \in H^{4,3}$. Here, the most singular terms are

$$
\begin{aligned}
& J_{1}=\left\|\frac{1}{4 \pi} \int_{-\infty}^{\infty} \int_{-\pi}^{\pi} F_{s}\left(s-s^{\prime}\right) \cos \left(\alpha^{\prime}\right)\left(\frac{\partial_{a} A^{\prime}[u]}{A^{\prime}[u]} \partial^{3} u_{\alpha}^{\prime}-\frac{\partial_{a} A^{\prime}[v]}{A^{\prime}[v]} \partial^{3} v_{\alpha}^{\prime}\right) d \alpha^{\prime} d s^{\prime}\right\|_{L^{2}} \\
& J_{2}=\left\|\frac{1}{4 \pi} \int_{-\infty}^{\infty} \int_{-\pi}^{\pi} F_{s}\left(s-s^{\prime}\right) \cos \left(\alpha^{\prime}\right)\left(\partial^{3}\left(\frac{\partial_{a} A^{\prime}[u]}{A^{\prime}[u]}\right) u_{\alpha}^{\prime}-\partial^{3}\left(\frac{\partial_{a} A^{\prime}[v]}{A^{\prime}[v]}\right) v_{\alpha}^{\prime}\right) d \alpha^{\prime} d s^{\prime}\right\|_{L^{2}} .
\end{aligned}
$$

In $J_{1}$ the most singular terms come from the differences

$$
\frac{\partial^{3} \partial_{a} A^{\prime}[u]}{A^{\prime}[u]} u_{\alpha}^{\prime}-\frac{\partial^{3} \partial_{a} A^{\prime}[v]}{A^{\prime}[v]} v_{\alpha}^{\prime}
$$

and

$$
\partial_{a} A^{\prime}[u] u_{\alpha}^{\prime} \partial^{3} \frac{1}{A^{\prime}[u]}-\partial_{a} A^{\prime}[v] v_{\alpha}^{\prime} \partial^{3} \frac{1}{A^{\prime}[v]}
$$

We will focus on

$$
J_{21}=\left\|\frac{1}{4 \pi} \int_{-\infty}^{\infty} \int_{-\pi}^{\pi} F_{s}\left(s-s^{\prime}\right) \cos \left(\alpha^{\prime}\right)\left(\frac{\partial^{3} \partial_{a} A^{\prime}[u]}{A^{\prime}[u]} u_{\alpha}^{\prime}-\frac{\partial^{3} \partial_{a} A^{\prime}[v]}{A^{\prime}[v]} v_{\alpha}^{\prime}\right) d \alpha^{\prime} d s^{\prime}\right\|_{L^{2}} .
$$

We will deal with this term by splitting

$$
\begin{aligned}
& \frac{u_{\alpha}^{\prime}}{A^{\prime}[u]} \partial^{3} \partial_{a} A^{\prime}[u]-\frac{v_{\alpha}^{\prime}}{A^{\prime}[v]} \partial^{3} \partial_{a} A^{\prime}[v] \\
& =\frac{u_{\alpha}^{\prime}}{A^{\prime}[u]}\left(\partial^{3} \partial_{a} A^{\prime}[u]-\partial^{3} \partial_{a} A^{\prime}[v]\right)+\partial^{3} \partial_{a} A^{\prime}[u]\left(\frac{u_{\alpha}^{\prime}}{A^{\prime}[u]}-\frac{v_{\alpha}^{\prime}}{A^{\prime}[v]}\right)
\end{aligned}
$$

We will give the details of the bound of

$$
J_{211}=\left\|\frac{1}{4 \pi} \int_{-\infty}^{\infty} \int_{-\pi}^{\pi} F_{s}\left(s-s^{\prime}\right) \cos \left(\alpha^{\prime}\right)\left(\frac{u_{\alpha}^{\prime}}{A[u]}\left(\partial^{3} \partial_{a} A^{\prime}[u]-\partial^{3} \partial_{a} A^{\prime}[v]\right)\right) d \alpha^{\prime} d s^{\prime}\right\|_{L^{2}} .
$$

We notice that

$$
\begin{aligned}
& \partial^{3} \partial_{a} A^{\prime}[u]-\partial^{3} \partial_{a} A^{\prime}[v]=8 a \partial^{3}\left(u+u^{\prime}\right) \sin ^{2}\left(\frac{\alpha^{\prime}}{2}\right)+4 a^{3}\left(4 \partial^{3}\left(u u^{\prime}\right) \sin ^{2}\left(\frac{\alpha^{\prime}}{2}\right)+\partial^{3}\left(u-u^{\prime}\right)^{2}\right) \\
& 8 a \partial^{3}\left(v+v^{\prime}\right) \sin ^{2}\left(\frac{\alpha^{\prime}}{2}\right)+4 a^{3}\left(4 \partial^{3}\left(v v^{\prime}\right) \sin ^{2}\left(\frac{\alpha^{\prime}}{2}\right)+\partial^{3}\left(v-v^{\prime}\right)^{2}\right)
\end{aligned}
$$

and the most singular terms here are

$$
\begin{aligned}
& 8 a \partial^{3}\left(u-v+u^{\prime}-v^{\prime}\right) \sin ^{2}\left(\frac{\alpha^{\prime}}{2}\right) \\
& +4 a^{3}\left(4\left(u^{\prime} \partial^{3} u-v^{\prime} \partial^{3} v+u \partial^{3} u^{\prime}-v \partial^{3} v^{\prime}\right) \sin ^{2}\left(\frac{\alpha^{\prime}}{2}\right)+2\left(u-u^{\prime}\right) \partial^{3}\left(u-u^{\prime}\right)-2\left(v-v^{\prime}\right) \partial^{3}\left(v-v^{\prime}\right)\right),
\end{aligned}
$$


and it is enough to consider the terms

$$
\begin{aligned}
& 8 a \partial^{3}\left(u-v+u^{\prime}-v^{\prime}\right) \sin ^{2}\left(\frac{\alpha^{\prime}}{2}\right) \\
& +4 a^{3}\left(4\left(u^{\prime} \partial^{3}(u-v)+u \partial^{3}\left(u^{\prime}-v^{\prime}\right)\right) \sin ^{2}\left(\frac{\alpha^{\prime}}{2}\right)+2\left(u-u^{\prime}\right) \partial^{3}\left(u-v-\left(u^{\prime}-v^{\prime}\right)\right)\right) .
\end{aligned}
$$

Then we have to estimate

$$
\begin{aligned}
& \mid \frac{u_{\alpha}^{\prime}}{A^{\prime}[u]}\left(8 a \partial^{3}\left(u-v+u^{\prime}-v^{\prime}\right) \sin ^{2}\left(\frac{\alpha^{\prime}}{2}\right)\right. \\
& \left.+4 a^{3}\left(4\left(u^{\prime} \partial^{3}(u-v)+u \partial^{3}\left(u^{\prime}-v^{\prime}\right)\right) \sin ^{2}\left(\frac{\alpha^{\prime}}{2}\right)+2\left(u-u^{\prime}\right) \partial^{3}\left(u-v-\left(u^{\prime}-v^{\prime}\right)\right)\right)\right) \mid \\
& \leq C a \frac{\sin ^{2}\left(\frac{\alpha^{\prime}}{2}\right)}{\sin ^{2}\left(\frac{\alpha^{\prime}}{2}\right)+b^{2} s^{\prime 2}}\left(\left|\partial^{3}(u-v)\right|+\left|\partial^{3}\left(u^{\prime}-v^{\prime}\right)\right|\right)+C a^{3} \frac{\sin ^{2}\left(\frac{\alpha^{\prime}}{2}\right)+s^{\prime 2}}{\sin ^{2}\left(\frac{\alpha^{\prime}}{2}\right)+b^{2} s^{\prime 2}}\left(\left|\partial^{3}(u-v)\right|+\left|\partial^{3}\left(u^{\prime}-v^{\prime}\right)\right|\right),
\end{aligned}
$$

where we have used lemma B.1 Then by applying lemma B.2 we can obtain a suitable estimate for $J_{211}$. The term $J_{2}$ can be bounded in a similar way.

This concludes the proof of lemma 3.3 .

Lemma 3.4 The derivative $\partial_{\tilde{r}} \mathcal{G}[\tilde{r}, a] g=\partial_{u} \mathcal{G}[u-s, a]$ is given by:

$$
\begin{aligned}
\partial_{u} \mathcal{G}[u-s, a] g & =a^{2} \lambda(a) g u_{\alpha}+\lambda(a)\left(1+a^{2} u\right) g_{\alpha} \\
& +\frac{a^{2} g}{4 \pi} \int_{-\infty}^{\infty} \int_{-\pi}^{\pi} F_{\rho}\left(s-s^{\prime}\right) \log \left(A^{\prime}\right) \cos \left(\alpha^{\prime}\right) u_{\alpha}^{\prime} d \alpha^{\prime} d s^{\prime} \\
& +\frac{1+a^{2} u}{4 \pi} \int_{-\infty}^{\infty} \int_{-\pi}^{\pi} F_{\rho}\left(s-s^{\prime}\right) \frac{\left(\partial_{u} A^{\prime}\right)[g]}{A^{\prime}} \cos \left(\alpha^{\prime}\right) u_{\alpha}^{\prime} d \alpha^{\prime} d s^{\prime} \\
& +\frac{1+a^{2} u}{4 \pi} \int_{-\infty}^{\infty} \int_{-\pi}^{\pi} F_{\rho}\left(s-s^{\prime}\right) \log \left(A^{\prime}\right) \cos \left(\alpha^{\prime}\right) g_{\alpha}^{\prime} d \alpha^{\prime} d s^{\prime} \\
& -\frac{g \alpha}{4 \pi} \int_{-\infty}^{\infty} \int_{-\pi}^{\pi} F_{\rho}\left(s-s^{\prime}\right) \log \left(A^{\prime}\right) \cos \left(\alpha^{\prime}\right)\left(1+a^{2} u^{\prime}\right) d \alpha^{\prime} d s^{\prime} \\
& -\frac{u_{\alpha}}{4 \pi} \int_{-\infty}^{\infty} \int_{-\pi}^{\pi} F_{\rho}\left(s-s^{\prime}\right) \frac{\left(\partial_{u} A^{\prime}\right)[g]}{A^{\prime}} \cos \left(\alpha^{\prime}\right)\left(1+a^{2} u^{\prime}\right) d \alpha^{\prime} d s^{\prime} \\
& -\frac{u_{\alpha}}{4 \pi} \int_{-\infty}^{\infty} \int_{-\pi}^{\pi} F_{\rho}\left(s-s^{\prime}\right) \log \left(A^{\prime}\right) \cos \left(\alpha^{\prime}\right) a^{2} g^{\prime} d \alpha^{\prime} d s^{\prime} \\
& +\frac{1}{4 \pi a^{2}} \int_{-\infty}^{\infty} \int_{-\pi}^{\pi} F_{\rho}\left(s-s^{\prime}\right) \frac{\left(\partial_{u} A^{\prime}\right)[g]}{A^{\prime}} \sin \left(\alpha^{\prime}\right) d \alpha^{\prime} d s^{\prime}, \\
& +\frac{1}{4 \pi} \int_{-\infty}^{\infty} \int_{-\pi}^{\pi} F_{\rho}\left(s-s^{\prime}\right) \frac{\left(\partial_{u} A^{\prime}\right)[g]}{A^{\prime}} \sin \left(\alpha^{\prime}\right)\left(u+u^{\prime}\right) d \alpha^{\prime} d s^{\prime}, \\
& +\frac{1}{4 \pi} \int_{-\infty}^{\infty} \int_{-\pi}^{\pi} F_{\rho}\left(s-s^{\prime}\right) \log \left(A^{\prime}\right) \sin \left(\alpha^{\prime}\right)\left(g+g^{\prime}\right) d \alpha^{\prime} d s^{\prime}, \\
& +\frac{a^{2}}{4 \pi} \int_{-\infty}^{\infty} \int_{-\pi}^{\pi} F_{\rho}\left(s-s^{\prime}\right) \frac{\left(\partial_{u} A^{\prime}\right)[g]}{A^{\prime}} \sin \left(\alpha^{\prime}\right)\left(u u^{\prime}+u_{\alpha} u_{\alpha}^{\prime}\right) d \alpha^{\prime} d s^{\prime}
\end{aligned}
$$




$$
+\frac{a^{2}}{4 \pi} \int_{-\infty}^{\infty} \int_{-\pi}^{\pi} F_{\rho}\left(s-s^{\prime}\right) \log \left(A^{\prime}\right) \sin \left(\alpha^{\prime}\right)\left(g u^{\prime}+g^{\prime} u+g_{\alpha} u_{\alpha}^{\prime}+g_{\alpha}^{\prime} u_{\alpha}\right) d \alpha^{\prime} d s^{\prime}
$$

Moreover this derivative is continuous in $H^{3}$.

Proof: We will focus in the derivatives of the terms $\mathcal{G}_{1}$ and $\mathcal{G}_{3}$ which are the most difficult ones.

In order to differentiate $\mathcal{G}_{1}$ with respect to $u$ we notice that thanks to lemma 3.1 in order to prove Lemma 3.4 is enough to compute the derivative of the term

$$
\mathcal{G}_{11}[u-s, a]=\frac{1}{4 \pi} \int_{-\infty}^{\infty} \int_{-\pi}^{\pi} F_{\rho}\left(s-s^{\prime}\right) \log \left(A^{\prime}\right) \cos \left(\alpha^{\prime}\right) u_{\alpha}^{\prime} d \alpha^{\prime} d s^{\prime} .
$$

Thus we need to show that

$$
\begin{aligned}
& \lim _{t \rightarrow 0} \frac{\mathcal{G}_{11}[u+t g-s, a]-\mathcal{G}_{11}[u-s, a]}{t}=\frac{1}{4 \pi} \int_{-\infty}^{\infty} \int_{-\pi}^{\pi} F_{\rho}\left(s-s^{\prime}\right) \log \left(A^{\prime}[u]\right) \cos \left(\alpha^{\prime}\right) g_{\alpha}^{\prime} d \alpha^{\prime} d s^{\prime} \\
& +\frac{1}{4 \pi} \int_{-\infty}^{\infty} \int_{-\pi}^{\pi} F_{\rho}\left(s-s^{\prime}\right) \frac{\partial_{u} A^{\prime}[g]}{A^{\prime}} \cos \left(\alpha^{\prime}\right) u_{\alpha}^{\prime} d \alpha^{\prime} d s^{\prime}
\end{aligned}
$$

is in $H^{3}$ for $g \in H^{3}$, with $\|g\|_{H^{3}}=1$, and that this derivative is continuous. We have that

$$
\begin{aligned}
& \mathcal{G}_{11}[u+t g-s, a]-\mathcal{G}_{11}[u-s, a] \\
& =\frac{1}{4 \pi} \int_{-\infty}^{\infty} \int_{-\pi}^{\pi} F_{\rho}\left(s-s^{\prime}\right) \cos \left(\alpha^{\prime}\right)\left(\log \left(A^{\prime}[u+t g]\right)\left(u^{\prime}+t g^{\prime}\right)_{\alpha}-\log \left(A^{\prime}[u]\right) u_{\alpha}^{\prime}\right) d \alpha^{\prime} d s^{\prime} .
\end{aligned}
$$

Taking 3 derivatives yields

$$
\begin{aligned}
& \partial^{3}\left(\mathcal{G}_{11}[u+t g-s, a]-\mathcal{G}_{11}[u-s, a]\right) \\
& =\sum_{j=0}^{3} \frac{c_{j}}{4 \pi} \int_{-\infty}^{\infty} \int_{-\pi}^{\pi} \cos \left(\alpha^{\prime}\right) \partial^{j}\left(\log \left(\frac{A^{\prime}[u+t g]}{A^{\prime}[u]}\right)\right) \partial^{3-j}\left(F_{\rho}\left(s-s^{\prime}\right) u_{\alpha}^{\prime}\right) d \alpha^{\prime} d s^{\prime} \\
& +t \sum_{j=0}^{3} \frac{c_{j}}{4 \pi} \int_{-\infty}^{\infty} \int_{-\pi}^{\pi} \cos \left(\alpha^{\prime}\right) \partial^{j}\left(\log \left(\frac{A^{\prime}[u+t g]}{A^{\prime}[u]}\right)\right) \partial^{3-j}\left(F_{\rho}\left(s-s^{\prime}\right) g_{\alpha}^{\prime}\right) d \alpha^{\prime} d s^{\prime} \\
& +t \sum_{j=0}^{3} \frac{c_{j}}{4 \pi} \int_{-\infty}^{\infty} \int_{-\pi}^{\pi} \cos \left(\alpha^{\prime}\right) \partial^{j}\left(\log \left(A^{\prime}[u]\right)\right) \partial^{3-j}\left(F_{\rho}\left(s-s^{\prime}\right) g_{\alpha}^{\prime}\right) d \alpha^{\prime} d s^{\prime} .
\end{aligned}
$$

Then it is enough to prove that

$$
\begin{aligned}
& \lim _{t \rightarrow 0}\left\|\int_{-\infty}^{\infty} \int_{-\pi}^{\pi} \cos \left(\alpha^{\prime}\right) \partial^{j}\left(\left(\frac{1}{t} \log \left(\frac{A^{\prime}[u+t g]}{A^{\prime}[u]}\right)-\frac{\partial_{u} A^{\prime}[g]}{A^{\prime}[u]}\right)\right) \partial^{3-j}\left(F_{\rho}\left(s-s^{\prime}\right) u_{\alpha}^{\prime}\right) d \alpha^{\prime} d s^{\prime}\right\|_{L^{2}}=0 \\
& \lim _{t \rightarrow 0}\left\|\int_{-\infty}^{\infty} \int_{-\pi}^{\pi} \cos \left(\alpha^{\prime}\right) \partial^{j}\left(\log \left(\frac{A^{\prime}[u+t g]}{A^{\prime}[u]}\right)\right) \partial^{3-j}\left(F_{\rho}\left(s-s^{\prime}\right) u_{\alpha}^{\prime}\right) d \alpha^{\prime} d s^{\prime}\right\|_{L^{2}}=0
\end{aligned}
$$

for all $j$. These equalities (3.4) and (3.5) are a consequence of lemmas B.22 and B.23,

For $\mathcal{G}_{3}$ we have that

$$
\mathcal{G}_{3}[u+t g-s, a]-\mathcal{G}_{3}[u-s, a]=\frac{1}{4 \pi} \int_{-\infty}^{\infty} \int_{-\pi}^{\pi} F_{\rho}\left(s-s^{\prime}\right) \frac{1}{b} \log \left(\frac{A^{\prime}[u+t g]}{A^{\prime}[u]}\right) \sin \left(\alpha^{\prime}\right) d \alpha^{\prime} d s^{\prime}
$$


and then we have to show that

$$
\lim _{t \rightarrow 0}\left\|\int_{-\infty}^{\infty} \int_{-\pi}^{\pi} \partial^{3-j} F_{\rho}\left(s-s^{\prime}\right) \frac{1}{b} \partial^{j}\left(\frac{1}{t} \log \left(\frac{A^{\prime}[u+t g]}{A^{\prime}[u]}\right)-\frac{\partial_{u} A^{\prime}[g]}{A^{\prime}[u]}\right) \sin \left(\alpha^{\prime}\right) d \alpha^{\prime} d s^{\prime}\right\|_{L^{2}}=0 .
$$

for $j=0,1,2,3$. This is also a consequence of lemmas B.24 and B.25

The proof of the the continuity in $H^{3}$ follows similar steps to those in lemma 3.3 ,

Lemma 3.5 The derivative $\partial_{u} \partial_{a} \mathcal{G}[u-s, a] g$ is given by:

$$
\begin{aligned}
& \partial_{u} \partial_{a} \mathcal{G}[u-s, a] g=a^{2} \frac{d \lambda}{d a}(a) g u_{\alpha}+\frac{d \lambda}{d a}(a)\left(1+a^{2} u\right) g_{\alpha}+2 a \lambda(a) g u_{\alpha}+2 a \lambda(a) u g_{\alpha} \\
& +\frac{a g}{2 \pi} \int_{-\infty}^{\infty} \int_{-\pi}^{\pi} F_{\rho}\left(s-s^{\prime}\right) \log \left(A^{\prime}\right) \cos \left(\alpha^{\prime}\right) u_{\alpha}^{\prime} d \alpha^{\prime} d s^{\prime} \\
& +\frac{a u}{2 \pi} \int_{-\infty}^{\infty} \int_{-\pi}^{\pi} F_{\rho}\left(s-s^{\prime}\right) \frac{\left(\partial_{u} A^{\prime}\right)[g]}{A^{\prime}} \cos \left(\alpha^{\prime}\right) u_{\alpha}^{\prime} d \alpha^{\prime} d s^{\prime} \\
& +\frac{a u}{2 \pi} \int_{-\infty}^{\infty} \int_{-\pi}^{\pi} F_{\rho}\left(s-s^{\prime}\right) \log \left(A^{\prime}\right) \cos \left(\alpha^{\prime}\right) g_{\alpha}^{\prime} d \alpha^{\prime} d s^{\prime} \\
& +\frac{a^{2} g}{4 \pi} \int_{-\infty}^{\infty} \int_{-\pi}^{\pi} F_{\rho}\left(s-s^{\prime}\right) \frac{\partial_{a} A^{\prime}}{A^{\prime}} \cos \left(\alpha^{\prime}\right) u_{\alpha}^{\prime} d \alpha^{\prime} d s^{\prime} \\
& +\frac{1+a^{2} u}{4 \pi} \int_{-\infty}^{\infty} \int_{-\pi}^{\pi} F_{\rho}\left(s-s^{\prime}\right)\left(\frac{\left(\partial_{u} \partial_{a}\right) A^{\prime}[g]}{A^{\prime}}-\frac{\partial_{a} A\left(\partial_{u} A\right)[g]}{A^{\prime 2}}\right) \cos \left(\alpha^{\prime}\right) u_{\alpha}^{\prime} d \alpha^{\prime} d s^{\prime} \\
& +\frac{1+a^{2} u}{4 \pi} \int_{-\infty}^{\infty} \int_{-\pi}^{\pi} F_{\rho}\left(s-s^{\prime}\right) \frac{\partial_{a} A^{\prime}}{A^{\prime}} \cos \left(\alpha^{\prime}\right) g_{\alpha}^{\prime} d \alpha^{\prime} d s^{\prime} \\
& -\frac{g_{\alpha}}{4 \pi} \int_{-\infty}^{\infty} \int_{-\pi}^{\pi} F_{\rho}\left(s-s^{\prime}\right) \frac{\partial_{a} A^{\prime}}{A^{\prime}} \cos \left(\alpha^{\prime}\right)\left(1+a^{2} u^{\prime}\right) d \alpha^{\prime} d s^{\prime} \\
& -\frac{u_{\alpha}}{4 \pi} \int_{-\infty}^{\infty} \int_{-\pi}^{\pi} F_{\rho}\left(s-s^{\prime}\right)\left(\frac{\left(\partial_{u} \partial_{a} A^{\prime}\right)[g]}{A^{\prime}}-\frac{\partial_{a} A^{\prime}\left(\partial_{u} A^{\prime}\right)[g]}{A^{\prime 2}}\right) \cos \left(\alpha^{\prime}\right)\left(1+a^{2} u^{\prime}\right) d \alpha^{\prime} d s^{\prime} \\
& -\frac{u_{\alpha}}{4 \pi} \int_{-\infty}^{\infty} \int_{-\pi}^{\pi} F_{\rho}\left(s-s^{\prime}\right) \frac{\partial_{a} A^{\prime}}{A^{\prime}} \cos \left(\alpha^{\prime}\right) a^{2} g^{\prime} d \alpha^{\prime} d s^{\prime} \\
& -\frac{a g_{\alpha}}{2 \pi} \int_{-\infty}^{\infty} \int_{-\pi}^{\pi} F_{\rho}\left(s-s^{\prime}\right) \log \left(A^{\prime}\right) \cos \left(\alpha^{\prime}\right) u^{\prime} d \alpha^{\prime} d s^{\prime} \\
& -\frac{a u_{\alpha}}{2 \pi} \int_{-\infty}^{\infty} \int_{-\pi}^{\pi} F_{\rho}\left(s-s^{\prime}\right) \frac{\left(\partial_{u} A^{\prime}\right)[g]}{A^{\prime}} \cos \left(\alpha^{\prime}\right) u^{\prime} d \alpha^{\prime} d s^{\prime} \\
& -\frac{a u_{\alpha}}{2 \pi} \int_{-\infty}^{\infty} \int_{-\pi}^{\pi} F_{\rho}\left(s-s^{\prime}\right) \log \left(A^{\prime}\right) \cos \left(\alpha^{\prime}\right) g^{\prime} d \alpha^{\prime} d s^{\prime} \\
& -\frac{1}{4 \pi} \int_{-\infty}^{\infty} \int_{-\pi}^{\pi} F_{\rho}\left(s-s^{\prime}\right) \partial_{a}\left(\frac{1}{a^{2}} \frac{\left(\partial_{u} A^{\prime}\right)[g]}{A^{\prime}}\right) \sin \left(\alpha^{\prime}\right) d \alpha^{\prime} d s^{\prime} \\
& +\frac{1}{4 \pi} \int_{-\infty}^{\infty} \int_{-\pi}^{\pi} F_{\rho}\left(s-s^{\prime}\right)\left(\frac{\left(\partial_{u} \partial_{a} A^{\prime}\right)[g]}{A^{\prime}}-\frac{\partial_{a} A^{\prime} \partial_{u} A^{\prime}[g]}{A^{\prime 2}}\right) \sin \left(\alpha^{\prime}\right)\left(u+u^{\prime}\right) d \alpha^{\prime} d s^{\prime} \\
& +\frac{1}{4 \pi} \int_{-\infty}^{\infty} \int_{-\pi}^{\pi} F_{\rho}\left(s-s^{\prime}\right) \frac{\partial_{a} A^{\prime}}{A^{\prime}} \sin \left(\alpha^{\prime}\right)\left(g+g^{\prime}\right) d \alpha^{\prime} d s^{\prime} \\
& +\frac{a^{2}}{4 \pi} \int_{-\infty}^{\infty} \int_{-\pi}^{\pi} F_{\rho}\left(s-s^{\prime}\right)\left(\frac{\left(\partial_{u} \partial_{a} A^{\prime}\right)[g]}{A^{\prime}}-\frac{\partial_{a} A^{\prime}\left(\partial_{u}\right) A^{\prime}[g]}{A^{\prime 2}}\right) \sin \left(\alpha^{\prime}\right)\left(u u^{\prime}+u_{\alpha} u_{\alpha}^{\prime}\right) d \alpha^{\prime} d s^{\prime}
\end{aligned}
$$




$$
\begin{aligned}
& +\frac{a^{2}}{4 \pi} \int_{-\infty}^{\infty} \int_{-\pi}^{\pi} F_{\rho}\left(s-s^{\prime}\right) \frac{\partial_{a} A^{\prime}}{A^{\prime}} \sin \left(\alpha^{\prime}\right)\left(g u^{\prime}+g^{\prime} u+g_{\alpha} u_{\alpha}^{\prime}+g_{\alpha}^{\prime} u\right) d \alpha^{\prime} d s^{\prime} \\
& +\frac{a}{2 \pi} \int_{-\infty}^{\infty} \int_{-\pi}^{\pi} F_{\rho}\left(s-s^{\prime}\right) \log \left(A^{\prime}\right) \sin \left(\alpha^{\prime}\right)\left(u g^{\prime}+g u^{\prime}+u_{\alpha} g_{\alpha}^{\prime}+u_{\alpha}^{\prime} g_{\alpha}\right) d \alpha^{\prime} d s^{\prime} \\
& +\frac{a}{2 \pi} \int_{-\infty}^{\infty} \int_{-\pi}^{\pi} F_{\rho}\left(s-s^{\prime}\right) \frac{\partial_{u} A^{\prime}[g]}{A^{\prime}} \sin \left(\alpha^{\prime}\right)\left(u u^{\prime}+u_{\alpha} u_{\alpha}^{\prime}\right) d \alpha^{\prime} d s^{\prime} .
\end{aligned}
$$

Moreover this derivative is continuous in $H^{3}$.

Proof: We will give the detail of the differentiation with respect to $u$ of the terms

$$
\partial_{a} \mathcal{G}_{1}[u-s, a]=\int_{-\infty}^{\infty} \int_{-\pi}^{\pi} F_{\rho}\left(s-s^{\prime}\right) \frac{\partial_{a} A^{\prime}}{A^{\prime}} \cos \left(\alpha^{\prime}\right) u_{\alpha}^{\prime} d \alpha^{\prime} d s^{\prime}
$$

and

$$
\partial_{a} \mathcal{G}_{2}[u-s, a]=\int_{-\infty}^{\infty} \int_{-\pi}^{\pi} F_{\rho}\left(s-s^{\prime}\right) \partial_{a}\left(\frac{1}{a^{2}} \log \left(\frac{A^{\prime}}{A^{\prime}[a=0]}\right)\right) \sin \left(\alpha^{\prime}\right) d \alpha^{\prime} d s^{\prime} .
$$

We will split $\partial_{a} \mathcal{G}_{2}$ into two terms $\partial_{a} \mathcal{G}_{2}=H_{1}+H_{2}$ with

$$
H_{1}=\int_{-\infty}^{\infty} \int_{-\pi}^{\pi} F_{\rho}\left(s-s^{\prime}\right)\left(-\frac{2}{a^{3}} \log \left(\frac{A^{\prime}[a]}{A^{\prime}[0]}\right)+\frac{2}{a}\left(u+u^{\prime}\right)\right) \sin \alpha^{\prime} d \alpha^{\prime} d s^{\prime}
$$

and

$$
H_{2}=\int_{-\infty}^{\infty} \int_{-\pi}^{\pi} F_{\rho}\left(s-s^{\prime}\right)\left(\frac{1}{a^{2}} \frac{\partial_{a} A^{\prime}[a]}{A^{\prime}[a]}-\frac{2}{a}\left(u+u^{\prime}\right)\right) \sin \alpha^{\prime} d \alpha^{\prime} d s^{\prime} .
$$

Next we differentiate $H_{2}$ with respect to $u$

$$
\begin{aligned}
& \frac{H_{2}[u+t g]-H_{2}[u]}{t} \\
& =\int_{-\infty}^{\infty} \int_{-\pi}^{\pi} F_{\rho}\left(s-s^{\prime}\right)\left(\frac{1}{a^{2} t} \frac{\partial_{a} A^{\prime}[u+t g]}{A^{\prime}[u+t g]}-\frac{1}{a^{2} t} \frac{\partial_{a} A^{\prime}[u]}{A^{\prime}[u]}-\frac{2}{a}\left(g+g^{\prime}\right)\right) \sin \left(\alpha^{\prime}\right) d \alpha^{\prime} d s^{\prime}
\end{aligned}
$$

In order to prove the lemma we need to show that

$$
\begin{aligned}
I \equiv & \int_{-\infty}^{\infty} \int_{-\pi}^{\pi} F_{\rho}\left(s-s^{\prime}\right)\left(\frac{1}{a^{2} t} \frac{\partial_{a} A^{\prime}[u+t g]}{A^{\prime}[u+t g]}-\frac{1}{a^{2} t} \frac{\partial_{a} A^{\prime}[u]}{A^{\prime}[u]}-\frac{2}{a}\left(g+g^{\prime}\right)\right) \sin \left(\alpha^{\prime}\right) d \alpha^{\prime} d s^{\prime} \\
& -\int_{-\infty}^{\infty} \int_{-\pi}^{\pi} F_{\rho}\left(s-s^{\prime}\right)\left(\frac{1}{a^{2}} \frac{\partial_{u} \partial_{a} A^{\prime}[g]}{A^{\prime}[u]}-\frac{1}{a^{2}} \frac{\partial_{a} A^{\prime}[g] \partial_{u} A^{\prime}[g]}{A^{\prime}[u]}-\frac{2}{a}\left(g+g^{\prime}\right)\right) \sin \left(\alpha^{\prime}\right) d \alpha^{\prime} d s^{\prime}
\end{aligned}
$$

tends to 0 in $H^{3}$. In order to check this fact we write

$$
\begin{aligned}
I= & \int_{-\infty}^{\infty} \int_{-\pi}^{\pi} F_{\rho}\left(s-s^{\prime}\right) \frac{1}{a^{2}}\left(\frac{1}{t} \frac{\partial_{a} A^{\prime}[u+t g]-\partial A^{\prime}[u]}{A^{\prime}[u+t g]}-\frac{\partial_{u} \partial_{a} A^{\prime}[g]}{A^{\prime}[u]}\right) \sin \left(\alpha^{\prime}\right) d s^{\prime} d \alpha^{\prime} \\
& +\int_{-\infty}^{\infty} \int_{-\pi}^{\pi} F_{\rho}\left(s-s^{\prime}\right) \frac{1}{a^{2}}\left(\frac{\partial_{a} A^{\prime}[u]}{t}\left(\frac{1}{A^{\prime}[u+t g]}-\frac{1}{A^{\prime}[u]}\right)+\frac{\partial_{a} A^{\prime}[g] \partial_{u} A^{\prime}[g]}{A^{\prime}[u]^{2}}\right) \sin \left(\alpha^{\prime}\right) d \alpha^{\prime} d s^{\prime} \\
& \equiv H_{21}+H_{22}
\end{aligned}
$$


Then

$$
\begin{aligned}
H_{21}= & \int_{-\infty}^{\infty} \int_{-\pi}^{\pi} F_{\rho}\left(s-s^{\prime}\right) \frac{1}{a^{2}}\left(\frac{1}{t} \frac{\partial_{a} A^{\prime}[u+t g]-\partial_{a} A^{\prime}[u]-t \partial_{u} \partial_{a} A^{\prime}[g]}{A^{\prime}[u+t g]}\right) \sin \left(\alpha^{\prime}\right) d \alpha d s^{\prime} \\
& \int_{-\infty}^{\infty} \int_{-\pi}^{\pi} F_{\rho}\left(s-s^{\prime}\right) \frac{1}{a^{2}} \frac{\partial_{u} \partial_{a} A^{\prime}[g]}{A^{\prime}[u+t g] A^{\prime}[u]}\left(A^{\prime}[u]-A^{\prime}[u+t g]\right) \sin \left(\alpha^{\prime}\right) d \alpha^{\prime} d s^{\prime} .
\end{aligned}
$$

Then we can apply lemma B.27 in order to prove that $H_{21} \rightarrow 0$ when $t \rightarrow 0$ in $H^{3}$. To show an analog result for $H_{22}$ we write

$$
\begin{aligned}
H_{22}= & \int_{-\infty}^{\infty} \int_{-\pi}^{\pi} F_{\rho}\left(s-s^{\prime}\right) \frac{1}{a^{2}} \frac{\partial_{a} A^{\prime}[g]}{t A^{\prime}[u] A^{\prime}[u+t g]}\left(A^{\prime}[u]-A^{\prime}[u+t g]+t \partial_{u} A^{\prime}[g]\right) \sin \left(\alpha^{\prime}\right) d \alpha^{\prime} d s^{\prime} \\
& +\int_{-\infty}^{\infty} \int_{-\pi}^{\pi} F_{\rho}\left(s-s^{\prime}\right) \frac{1}{a^{2}} \frac{\partial_{a} A^{\prime}[g] \partial_{u} A^{\prime}[g]}{A^{\prime}[u+t g] A^{\prime}[u]^{2}}\left(A^{\prime}[u+t g]-A^{\prime}[u]\right) \sin \left(\alpha^{\prime}\right) d \alpha^{\prime} d s^{\prime} .
\end{aligned}
$$

and apply lemma B.27

In order to differentiate $H_{1}$ we proceed as follows

$$
\frac{H_{1}[u+t g]-H_{1}[u]}{t}=\int_{-\infty}^{\infty} \int_{-\pi}^{\pi} F_{s}\left(s-s^{\prime}\right)\left(-\frac{2}{a^{3}} \frac{1}{t} \log \left(\frac{A^{\prime}[u+t g]}{A^{\prime}[u]}\right)+\frac{2}{a}\left(g+g^{\prime}\right)\right) \sin \left(\alpha^{\prime}\right) d \alpha^{\prime} d s^{\prime}
$$

And we would like to show that

$$
\int_{-\infty}^{\infty} \int_{-\pi}^{\pi} F_{s}\left(s-s^{\prime}\right)\left(-\frac{2}{a^{3}} \frac{1}{t} \log \left(\frac{A^{\prime}[u+t g]}{A^{\prime}[u]}\right)+\frac{2}{a^{3}} \frac{\partial_{u} A^{\prime}[u]}{A^{\prime}[u]}\right) \sin \left(\alpha^{\prime}\right) d \alpha^{\prime} d s^{\prime}
$$

tends to 0 , as $t$ goes to 0 in $H^{3}$. In order to do it we apply lemma B.26

Finally we differentiate $\partial_{a} G_{1}[u-s, a]$ with respect to $u$. Let us call $J[u] \equiv \partial_{a} G_{1}[u-s, a]$. We have that

$$
\begin{aligned}
\frac{J[u+t g]-J[u]}{t}= & \frac{1}{t} \int_{-\infty}^{\infty} \int_{-\pi}^{\pi} F_{s}\left(s-s^{\prime}\right) \frac{\partial_{a} A^{\prime}[u+t g]}{A^{\prime}[u+t g]} \cos \left(\alpha^{\prime}\right)\left(u_{\alpha}^{\prime}+t g_{\alpha}^{\prime}\right) d \alpha^{\prime} d s^{\prime} \\
& -\frac{1}{t} \int_{-\infty}^{\infty} \int_{-\pi}^{\pi} F_{s}\left[s-s^{\prime}\right] \frac{A^{\prime}[u+t g]}{A^{\prime}[u]} \cos \left(\alpha^{\prime}\right) d \alpha^{\prime} d s^{\prime} \\
& =\int_{-\infty}^{\infty} \int_{-\pi}^{\pi} F_{s}\left(s-s^{\prime}\right) \frac{1}{t}\left(\frac{\partial_{a} A^{\prime}[u+t g]}{A^{\prime}[u+t g]}-\frac{\partial_{a} A^{\prime}[u]}{A^{\prime}[u]}\right) \cos \left(\alpha^{\prime}\right)\left(u_{\alpha}^{\prime}+t g_{\alpha}^{\prime}\right) d \alpha^{\prime} d s^{\prime} \\
& +\int_{-\infty}^{\infty} \int_{-\pi}^{\pi} F_{s}\left(s-s^{\prime}\right) \frac{\partial_{a} A^{\prime}[u+t g]}{A^{\prime}[u+t g]} \cos \left(\alpha^{\prime}\right) g_{\alpha}^{\prime} d \alpha^{\prime} d s^{\prime} .
\end{aligned}
$$

We need to show that

$$
\begin{aligned}
& \frac{J[u+t g]-J[u]}{t}-\int_{-\infty}^{\infty} \int_{-\pi}^{\pi} F_{s}\left(s-s^{\prime}\right)\left(\frac{\partial_{u} \partial_{a} A^{\prime}[u]}{A^{\prime}[u]}-\frac{\partial_{a} A^{\prime} \partial_{u} A^{\prime}[u]}{A^{\prime}[u]^{2}}\right) \cos \left(\alpha^{\prime}\right) u_{\alpha}^{\prime} d \alpha^{\prime} d s^{\prime} \\
& -\int_{-\infty}^{\infty} \int_{-\pi}^{\pi} F_{s}\left(s-s^{\prime}\right) \frac{\partial_{a} A^{\prime}[u]}{A^{\prime}[u]} \cos \left(\alpha^{\prime}\right) g_{\alpha}^{\prime} d \alpha^{\prime} d s^{\prime},
\end{aligned}
$$

tends to 0 , when $t$ goes to 0 in $H^{3}$. This means that

$$
\int_{-\infty}^{\infty} \int_{-\pi}^{\pi} F_{s}\left(s-s^{\prime}\right)\left(\frac{1}{t}\left(\frac{\partial_{a} A^{\prime}[u+t g]}{A^{\prime}[u+t g]}-\frac{\partial A^{\prime}[u]}{A^{\prime}[u]}\right)-\frac{\partial_{u} \partial_{a} A^{\prime}[u]}{A^{\prime}[u]}+\frac{\partial_{a} A^{\prime}[u] \partial_{u} A^{\prime}[u]}{A^{\prime}[u]^{2}}\right) \cos \left(\alpha^{\prime}\right) u_{\alpha}^{\prime} d \alpha^{\prime} d s^{\prime}
$$




$$
\int_{-\infty}^{\infty} \int_{-\pi}^{\pi} F_{s}\left(s-s^{\prime}\right)\left(\frac{\partial_{a} A^{\prime}[u+t g]}{A^{\prime}[u+t g]}-\frac{\partial_{a} A^{\prime}[u]}{A^{\prime}[u]}\right) \cos \left(\alpha^{\prime}\right) g_{\alpha}^{\prime} d \alpha^{\prime} d s^{\prime}
$$

tends to 0 when $t$ goes to 0 in $H^{3}$. Thus, it is enough to prove that, in $H^{3}$,

$\lim _{t \rightarrow 0} \int_{-\infty}^{\infty} \int_{-\pi}^{\pi} F_{s}\left(s-s^{\prime}\right)\left(\frac{1}{t}\left(\frac{\partial_{a} A^{\prime}[u+t g]}{A^{\prime}[u+t g]}-\frac{\partial A^{\prime}[u]}{A^{\prime}[u]}\right)-\frac{\partial_{u} \partial_{a} A^{\prime}[u]}{A^{\prime}[u]}+\frac{\partial_{a} A^{\prime}[u] \partial_{u} A^{\prime}[u]}{A^{\prime}[u]^{2}}\right) \cos \left(\alpha^{\prime}\right) u_{\alpha}^{\prime} d \alpha^{\prime} d s^{\prime}=0$.

In order to prove (3.7) we will consider two terms separately

$$
J_{1}=\int_{-\infty}^{\infty} \int_{-\pi}^{\pi} F_{s}\left(s-s^{\prime}\right)\left(\frac{1}{t} \frac{\partial_{a} A^{\prime}[u+t g]-\partial_{a} A^{\prime}[u]}{A^{\prime}[u+t g]}-\frac{\partial_{u} \partial_{a} A^{\prime}[u]}{A^{\prime}[u]}\right) \cos \left(\alpha^{\prime}\right) u_{\alpha}^{\prime} d \alpha^{\prime} d s^{\prime}
$$

and

$$
J_{2}=\int_{-\infty}^{\infty} \int_{-\pi}^{\pi} F_{s}\left(s-s^{\prime}\right) \partial_{a} A^{\prime}[u]\left(\frac{1}{t}\left(\frac{1}{A^{\prime}[u+t g]}-\frac{1}{A^{\prime}[u]}\right)+\frac{\partial_{u} A^{\prime}[u]}{A^{\prime}[u]^{2}}\right)
$$

In addition we will split $J_{1}$ into two terms

$$
\begin{aligned}
J_{1}= & \int_{-\infty}^{\infty} \int_{-\pi}^{\pi} F_{s}\left(s-s^{\prime}\right) \frac{1}{t}\left(\frac{\partial_{a} A^{\prime}[u+t g]-\partial_{a} A^{\prime}[u]-t \partial_{u} \partial_{a} A^{\prime}[u]}{A[u+t g]}\right) \cos \left(\alpha^{\prime}\right) u_{\alpha}^{\prime} d \alpha^{\prime} d s^{\prime} \\
& +\int_{-\infty}^{\infty} \int_{-\pi}^{\pi} F_{s}\left(s-s^{\prime}\right) \partial_{u} \partial_{a} A^{\prime}[u+t g]\left(\frac{1}{A^{\prime}[u+t g]}-\frac{1}{A^{\prime}[u]}\right) \cos \left(\alpha^{\prime}\right) u_{\alpha}^{\prime} d \alpha^{\prime} d s^{\prime} \\
\equiv & J_{11}+J_{12} .
\end{aligned}
$$

The term $J_{11}$ and $J_{12}$ can be controlled by using B.28,

In order to deal with $J_{2}$ we also split it into two parts

$$
\begin{aligned}
J_{2}= & \int_{-\infty}^{\infty} \int_{-\pi}^{\pi} f_{s}\left(s-s^{\prime}\right) \partial_{a} A^{\prime}[u] \frac{1}{t} \frac{A^{\prime}[u]-A^{\prime}[u+t g]+t \partial_{u} A^{\prime}[u]}{A^{\prime}[u+t g] A^{\prime}[u]} \cos \left(\alpha^{\prime}\right) u_{\alpha}^{\prime} d \alpha^{\prime} d s^{\prime} \\
& +\int_{-\infty}^{\infty} \int_{-\pi}^{\pi} F_{s}\left(s-s^{\prime}\right) \frac{\partial_{a} A^{\prime}[u] \partial_{u} A^{\prime}[u]}{A^{\prime}[u]}\left(\frac{1}{A^{\prime}[u+t g]}-\frac{1}{A^{\prime}[u]}\right) \cos \left(\alpha^{\prime}\right) u_{\alpha}^{\prime} d \alpha^{\prime} d s^{\prime} \\
\equiv & J_{21}+J_{22} .
\end{aligned}
$$

We control the terms $J_{21}$ and $J_{22}$ by using the lemma B.28

The proof of the the continuity in $H^{3}$ follows similar steps to those in lemma 3.3 .

This concludes the proof of lemma 3.5.

\subsection{Step 3. Analysis of the linear part.}

\subsubsection{One dimensionality of the Kernel of the linear operator.}

We will start computing a nontrivial element of the kernel of $\partial_{\tilde{r}} \mathcal{G}[0,0] g=0$, which is given by:

$$
\begin{aligned}
& \partial_{\tilde{r}} \mathcal{G}[0,0] g=\lambda(0) g_{\alpha}(\alpha, s) \\
& +\frac{1}{4 \pi} \int_{-\pi}^{\pi} \int_{-1}^{1} F_{\rho}\left(s^{\prime}\right) \log \left(2-2 \cos \left(\alpha-\alpha^{\prime}\right)\right) \cos \left(\alpha-\alpha^{\prime}\right) g_{\alpha}\left(\alpha^{\prime}, s^{\prime}\right) d \alpha^{\prime} d s^{\prime} \\
& -\frac{g_{\alpha}(\alpha, s)}{4 \pi} \int_{-\pi}^{\pi} \int_{-1}^{1} F_{\rho}\left(s^{\prime}\right) \log \left(2-2 \cos \left(\alpha-\alpha^{\prime}\right)\right) \cos \left(\alpha-\alpha^{\prime}\right) d \alpha^{\prime} d s^{\prime}
\end{aligned}
$$




$$
\begin{aligned}
& +\frac{1}{4 \pi} \int_{-\pi}^{\pi} \int_{-1}^{1} F_{\rho}\left(s^{\prime}\right) \sin \left(\alpha-\alpha^{\prime}\right) g\left(\alpha^{\prime}, s^{\prime}\right) d \alpha^{\prime} d s^{\prime} \\
& +\frac{1}{4 \pi} \int_{-\pi}^{\pi} \int_{-1}^{1} F_{\rho}\left(s^{\prime}\right) \sin \left(\alpha-\alpha^{\prime}\right) \log \left(2-2 \cos \left(\alpha-\alpha^{\prime}\right)\right) g\left(\alpha^{\prime}, s^{\prime}\right) d \alpha^{\prime} d s^{\prime}
\end{aligned}
$$

We make the decomposition

$$
g(\alpha, s)=\sum_{n=1}^{\infty} g_{n}(s) \sin (n \alpha)
$$

and project onto the $m$-th mode. For each $m$ we get the following system of equations:

$$
\begin{aligned}
0 & =-m g_{m}(s) \lambda(0) \sin (m \alpha) \\
& -\frac{m}{4 \pi} \int_{-\pi}^{\pi} \int_{-1}^{1} F_{\rho}\left(s^{\prime}\right) \log \left(2-2 \cos \left(\alpha-\alpha^{\prime}\right)\right) \cos \left(\alpha-\alpha^{\prime}\right) g_{m}\left(s^{\prime}\right) \sin \left(m \alpha^{\prime}\right) d \alpha^{\prime} d s^{\prime} \\
& +\frac{m g_{m}(s) \sin (m \alpha)}{4 \pi} \int_{-\pi}^{\pi} \int_{-1}^{1} F_{\rho}\left(s^{\prime}\right) \log \left(2-2 \cos \left(\alpha-\alpha^{\prime}\right)\right) \cos \left(\alpha-\alpha^{\prime}\right) d \alpha^{\prime} d s^{\prime} \\
& +\frac{1}{4 \pi} \int_{-\pi}^{\pi} \int_{-1}^{1} F_{\rho}\left(s^{\prime}\right) \sin \left(\alpha-\alpha^{\prime}\right) g_{m}\left(s^{\prime}\right) \cos \left(m \alpha^{\prime}\right) d \alpha^{\prime} d s^{\prime} \\
& +\frac{1}{4 \pi} \int_{-\pi}^{\pi} \int_{-1}^{1} F_{\rho}\left(s^{\prime}\right) g_{m}\left(s^{\prime}\right) \sin \left(\alpha-\alpha^{\prime}\right) \log \left(2-2 \cos \left(\alpha-\alpha^{\prime}\right)\right) g_{m}\left(s^{\prime}\right) \cos \left(m \alpha^{\prime}\right) d \alpha^{\prime} d s^{\prime} \\
& =-m g_{m}(s) \lambda(0) \sin (m \alpha) \\
& -\frac{m}{4 \pi} \int_{-1}^{1} F_{\rho}\left(s^{\prime}\right) g_{m}\left(s^{\prime}\right) \int_{-\pi}^{\pi} \log \left(2-2 \cos \left(\alpha-\alpha^{\prime}\right)\right) \cos \left(\alpha-\alpha^{\prime}\right) \sin \left(m \alpha^{\prime}\right) d \alpha^{\prime} d s^{\prime} \\
& +\frac{m g_{m}(s) \sin (m \alpha)}{4 \pi} \int_{-1}^{1} F_{\rho}\left(s^{\prime}\right) \int_{-\pi}^{\pi} \log \left(2-2 \cos \left(\alpha-\alpha^{\prime}\right)\right) \cos \left(\alpha-\alpha^{\prime}\right) d \alpha^{\prime} d s^{\prime} \\
& +\frac{1}{4 \pi} \int_{-1}^{1} F_{\rho}\left(s^{\prime}\right) g_{m}\left(s^{\prime}\right) \int_{-\pi}^{\pi} \sin \left(\alpha-\alpha^{\prime}\right) \cos \left(m \alpha^{\prime}\right) d \alpha^{\prime} d s^{\prime} \\
& +\frac{1}{4 \pi} \int_{-1}^{1} F_{\rho}\left(s^{\prime}\right) g_{m}\left(s^{\prime}\right) \int_{-\pi}^{\pi} \sin \left(\alpha-\alpha^{\prime}\right) \log \left(2-2 \cos \left(\alpha-\alpha^{\prime}\right)\right) \cos \left(m \alpha^{\prime}\right) d \alpha^{\prime} d s^{\prime} \\
& =-m g_{m}(s) \lambda(0)-\frac{m}{4 \pi}(-2 \pi) \frac{m}{m^{2}-1} \int_{-1}^{1} F_{\rho}\left(s^{\prime}\right) g_{m}\left(s^{\prime}\right) d s^{\prime} \\
& +\frac{m g_{m}(s)}{4 \pi}(-2 \pi) \int_{-1}^{1} F_{\rho}\left(s^{\prime}\right) d s^{\prime}+\frac{1}{4 \pi}(-2 \pi) \frac{1}{m^{2}-1} \int_{-1}^{1} F_{\rho}\left(s^{\prime}\right) g_{m}\left(s^{\prime}\right) d s^{\prime} \\
& =-m g_{m}(s) \lambda(0)+\frac{1}{2} \int_{-1}^{1} F_{\rho}\left(s^{\prime}\right) g_{m}\left(s^{\prime}\right) d s^{\prime}-\frac{m g_{m}(s)}{2} F_{\rho}^{1}\left(s^{\prime}\right) d s^{\prime} .
\end{aligned}
$$

We will use now the following lemma:

Lemma 3.6 Let $m \in \mathbb{N}$ and $F_{\rho}(s)$ be such that $\int_{-1}^{1} F_{\rho}(s) d s=-1$. Then, for $k \in \mathbb{N}$, the equation 


$$
g_{k}(s)=-\frac{m}{k} \int_{-1}^{1} F_{\rho}\left(s^{\prime}\right) g_{k}\left(s^{\prime}\right) d s^{\prime}
$$

has solutions:

$$
g_{k}(s)= \begin{cases}c & \text { if } k=m \\ 0 & \text { if } k \neq m\end{cases}
$$

where $c$ is any real number.

Proof: Since the RHS does not depend on $s$, the only possible solutions are the constant ones. Setting $g_{k}(s)=c$ and substituting into the equation yields the result.

Rearranging terms and using the fact that $\lambda(0)=\frac{m-1}{2 m}$ and that $F_{\rho}$ has integral equal to -1 , we are left to solve

$$
g_{k}(s)=-\frac{m}{k} \int_{-1}^{1} F_{\rho}\left(s^{\prime}\right) g_{k}\left(s^{\prime}\right) d s^{\prime}
$$

By the lemma, there exists a nontrivial solution only whenever $k=m$, and in that case, it is given by a constant function $c$. This shows the existence of a nontrivial kernel of dimension 1 .

\subsubsection{Codimension of the image of the linear operator.}

We now characterize the image of $\partial_{\tilde{r}} \mathcal{G}[0,0]$. We have the following Lemma:

Lemma 3.7 Let

$$
Z=\left\{q(s, \alpha) \in H_{m, o d d}^{3,3}, q(s, \alpha)=\sum_{k=1}^{\infty} q_{k}(s) \sin (k \alpha), \int_{-1}^{1} F_{\rho}\left(s^{\prime}\right) q_{m}\left(s^{\prime}\right) d s^{\prime}=0\right\}
$$

Then $Z=\operatorname{Im}\left(\partial_{\tilde{r}} \mathcal{G}[0,0]\right)$.

Proof:

We start proving that $\operatorname{Im}\left(\partial_{\tilde{r}} \mathcal{G}[0,0]\right) \subset Z$. This follows easily from (3.8) since the $m$-th mode of $\operatorname{Im}\left(\partial_{\tilde{r}} \mathcal{G}[0,0]\right) g$ is given by

$$
\frac{g_{m}(s)}{2}+\frac{1}{2} \int_{-1}^{1} F_{\rho}\left(s^{\prime}\right) g_{m}\left(s^{\prime}\right) d s^{\prime}
$$

yielding zero after multiplying by $F(s)$ and integrating over $[-1,1]$.

We now prove the other implication and show that $Z \subset \operatorname{Im}\left(\partial_{\tilde{r}} \mathcal{G}[0,0]\right)$.

Let $q(s, \alpha) \in Z$. We want to show that there exists a $g(s, \alpha) \in H_{m, \text { even }}^{4,3}$ such that $\partial_{\tilde{r}} \mathcal{G}[0,0] g=q$. It is enough to show that $g(s, \alpha) \in H^{4,3}$ since the $m$-fold property and the evenness follow trivially. Let us project $g$ into Fourier modes as 


$$
g(s, \alpha)=\sum_{k=m, k \mid m}^{\infty} g_{k}(s) \cos (k \alpha), \quad q(s, \alpha)=\sum_{k=m, k \mid m}^{\infty} q_{k}(s) \sin (k \alpha) .
$$

This yields the following system of equations:

$$
q_{k}(s)=k g_{k}(s) \frac{1-m}{2 m}+\frac{1}{2} \int_{-1}^{1} F_{\rho}\left(s^{\prime}\right) g_{k}\left(s^{\prime}\right) d s^{\prime}+\frac{k g_{k}(s)}{2},
$$

which implies that $g_{k}(s)$ has to be of the form $g_{k}(s)=A_{k} q_{k}(s)+B_{k}$ for some constants $A_{k}, B_{k}$. We distinguish two cases.

If $k=m$, then equation (3.9) yields:

$$
\begin{aligned}
q_{m}(s) & =g_{m}(s) \frac{1}{2}+\frac{1}{2} \int_{-1}^{1} F_{\rho}\left(s^{\prime}\right) g_{m}\left(s^{\prime}\right) d s^{\prime} \\
& =\frac{A_{m}}{2} q_{m}(s)+\frac{1}{2} \int_{-1}^{1} F_{\rho}\left(s^{\prime}\right) q_{m}\left(s^{\prime}\right),
\end{aligned}
$$

and the only solution is $A_{m}=2, B_{m} \in \mathbb{R}$, under the additional constraint

$$
\int_{-1}^{1} F_{\rho}\left(s^{\prime}\right) q_{m}\left(s^{\prime}\right)=0
$$

and no solution otherwise. If $k \neq m$, then we have to solve

$$
q_{k}(s)=q_{k}(s) \frac{k A_{k}}{2 m}+\frac{k B_{k}}{2 m}+\frac{A_{k}}{2} \int_{-1}^{1} F_{\rho}\left(s^{\prime}\right) q_{k}\left(s^{\prime}\right) d s^{\prime}-\frac{B_{k}}{2},
$$

which has a unique solution

$$
A_{k}=\frac{2 m}{k}, \quad B_{k}=\frac{2 m^{2}}{k(m-k)} \int_{-1}^{1} F_{\rho}\left(s^{\prime}\right) q_{k}\left(s^{\prime}\right) d s^{\prime},
$$

for any $q_{k}(s)$. This shows the existence and the uniqueness. We discuss the regularity now. To do so, we compute the $H^{4,3}$ norm of $g(s, \alpha)$ :

$$
\begin{aligned}
&\|g\|_{H^{4,3}}^{2} \lesssim \sum_{k=1}^{\infty}\left\|g_{k}\right\|_{H^{3}}^{2}\left(1+k^{4}\right)^{2} \leq \sum_{k=1}^{\infty}\left\|q_{k}\right\|_{H^{3}}^{2} \frac{4 m^{2}}{k^{2}}\left(1+k^{4}\right)^{2}+C \sum_{k=1, k \neq m}^{\infty} \frac{m^{4}}{k^{2}(m-k)^{2}}\left\|q_{k}\right\|_{L^{2}}^{2}\left(1+k^{4}\right)^{2} \\
& \lesssim \sum_{k=1}^{\infty}\left\|q_{k}\right\|_{H^{3}}^{2}\left(1+k^{3}\right)^{2} \\
& \lesssim\|q\|_{H^{3,3}}^{2} .
\end{aligned}
$$

This concludes that $Z=\operatorname{Im}\left(\partial_{\tilde{r}} \mathcal{G}[0,0]\right)$ and in particular shows that the codimension of the image of $\partial_{\tilde{r}} \mathcal{G}[0,0]$ is 1 , as we needed. 


\subsection{Step 4. The transversality property 4 .}

This step is devoted to show the transversality condition. We start writing out the calculations since everything is explicit, including the characterization of the image done in the previous subsection. Based on that, we only need to compute the $m$-th Fourier coefficient of $\partial_{\tilde{r}} \partial_{a} \mathcal{G}[\tilde{r}, a] g$.

By setting $a=0, \tilde{r}=0$ in (3.6), one obtains:

$$
\left.\partial_{\tilde{r}} \partial_{a} \mathcal{G}[\tilde{r}, a] g\right|_{\tilde{r}=0, a=0}=\frac{d \lambda}{d a}(0) g_{\alpha}
$$

We now take $g(\alpha, s)=\cos (m \alpha)$ and extract the contribution to the $m$-th mode. We get that it is equal to

$$
-m \frac{d \lambda}{d a}(0) \sin (m \alpha)
$$

Testing the coefficient in front of $\sin (m \alpha)$ against $F_{\rho}(s)$ and integrating in $[-1,1]$ we obtain

$$
m \frac{d \lambda}{d a}(0)
$$

which is different than 0 as long as $\frac{d \lambda}{d a}(0) \neq 0$, thus satisfying the transversality condition.

\section{Acknowledgements}

AC, DC and JGS were partially supported by the grant MTM2014-59488-P (Spain) and ICMAT Severo Ochoa project SEV-2015-556. AC was partially supported by the Ramón y Cajal program RyC-2013-14317 and ERC grant 307179-GFTIPFD. JGS was partially supported by an AMS-Simons Travel Grant. Part of this work was done while JGS was visiting ICMAT, to which he is grateful for its support. We thank Jacob Bedrossian and Vlad Vicol for helpful conversations.

\section{A Basic integrals}

In this section we will outline the basic integral identities used in the spectral analysis of subsection 3.3

The first three lemmas follow easily from [9, Lemma A.3]

\section{Lemma A.1}

$$
\int_{-\pi}^{\pi} \log \left(2-2 \cos \left(\alpha^{\prime}\right)\right) \cos \left(\alpha^{\prime}\right) d \alpha^{\prime}=-2 \pi
$$

Lemma A.2 Let $m$ be a positive integer. Then:

$$
\int_{-\pi}^{\pi} \log \left(2-2 \cos \left(\alpha^{\prime}\right)\right) \cos \left(\alpha^{\prime}\right) \cos \left(m \alpha^{\prime}\right) d \alpha^{\prime}=\left\{\begin{array}{cc}
(-2 \pi) \frac{m}{m^{2}-1} & \text { if } m \neq 1 \\
-\frac{\pi}{2} & \text { if } m=1
\end{array}\right.
$$


Lemma A.3 Let $m$ be a positive integer. Then:

$$
\int_{-\pi}^{\pi} \log \left(2-2 \cos \left(\alpha^{\prime}\right)\right) \sin \left(\alpha^{\prime}\right) \sin \left(m \alpha^{\prime}\right) d \alpha^{\prime}=\left\{\begin{array}{cc}
(-2 \pi) \frac{1}{m^{2}-1} & \text { if } m \neq 1 \\
\frac{\pi}{2} & \text { if } m=1
\end{array}\right.
$$

The next two integrals are obvious but we state them here for convenience:

Lemma A.4 Let $m$ be a positive integer. Then:

$$
\int_{-\pi}^{\pi} \cos \left(\alpha^{\prime}\right) \cos \left(m \alpha^{\prime}\right) d \alpha^{\prime}= \begin{cases}0 & \text { if } m \neq 1 \\ \pi & \text { if } m=1\end{cases}
$$

Lemma A.5 Let $m$ be a positive integer. Then:

$$
\int_{-\pi}^{\pi} \sin \left(\alpha^{\prime}\right) \sin \left(m \alpha^{\prime}\right) d \alpha^{\prime}= \begin{cases}0 & \text { if } m \neq 1 \\ \pi & \text { if } m=1\end{cases}
$$

\section{B Estimates on $A^{\prime}$}

In this section we will assume the following:

1. The function $\tilde{r}=u-s \in V^{\delta}$, i.e. $\|\tilde{r}\|_{H^{4,3}} \leq \delta$.

2. $C(\delta)$ will denote a finite positive constant that just depends on $\delta$, that increases with $\delta$ and such that $C(0)=0$. Also $c(\delta)$ will be a constant that just depends on $\delta$ and such that $c(\delta) \geq \bar{c}>0$ for all $\delta \in\left[0, \delta_{0}\right]$ and $\delta_{0}$ small enough.

3. The parameter $a \in\left[-a_{0}, a_{0}\right]$, for some small enough $a_{0}$ and $\delta \in\left[0, \delta_{0}\right]$ for some small enough $\delta_{0}$.

4. $C$ and $c$ will be constants such that $C<\infty$ and $c>0$.

5. $\partial$ means either $\partial_{\alpha}$ or $\partial_{s}$.

We also recall that given any function $f(\alpha, s)$ we will write

$$
\begin{aligned}
f & =f(\alpha, s) \\
f^{\prime} & =f\left(\alpha-\alpha^{\prime}, s-s^{\prime}\right) .
\end{aligned}
$$

We want to obtain estimates on $\mathcal{G}[u-s, a]$ in (2.10). In order to do it we will set $b=a^{2}$.

Lemma B.1 The function

$$
A^{\prime}=4 \sin ^{2}\left(\frac{\alpha^{\prime}}{2}\right)+4 b\left(u+u^{\prime}\right) \sin ^{2}\left(\frac{\alpha^{\prime}}{2}\right)+b^{2}\left(4 u u^{\prime} \sin ^{2}\left(\frac{\alpha^{\prime}}{2}\right)+\left(u-u^{\prime}\right)^{2}\right)
$$

satisfies

1.

$$
A^{\prime} \geq c\left(\sin ^{2}\left(\frac{\alpha^{\prime}}{2}\right)+b^{2} s^{\prime 2}\right)
$$

2.

$$
\log \left(A^{\prime}\right) \leq \log \left(\sin ^{2}\left(\frac{\alpha^{\prime}}{2}\right)+b^{2} s^{\prime 2}\right)+C,
$$


3.

$$
\partial A^{\prime} \leq C b\left(\sin ^{2}\left(\frac{\alpha^{\prime}}{2}\right)+b s^{\prime 2}\right)
$$

4.

$$
\partial^{2} A^{\prime} \leq C b\left(\sin ^{2}\left(\frac{\alpha^{\prime}}{2}\right)+b\left(\left|\sin \left(\frac{\alpha^{\prime}}{2}\right)\right|+\left|s^{\prime}\right|+s^{\prime 2}\right)\right)
$$

Proof: Using that $\|\tilde{r}\|_{H^{4,3}} \leq \delta$ and the Sobolev embedding we have that

$$
\left|A^{\prime}\right| \geq 4 \sin ^{2}\left(\frac{\alpha^{\prime}}{2}\right)-b C(\delta) \sin ^{2}\left(\frac{\alpha^{\prime}}{2}\right)-b^{2} C(\delta) \sin ^{2}\left(\frac{\alpha^{\prime}}{2}\right)+b^{2}\left(u-u^{\prime}\right)^{2} .
$$

In addition,

$$
\begin{aligned}
& \left(u-u^{\prime}\right)^{2}=\left(s^{\prime}+\tilde{r}-\tilde{r}^{\prime}\right)^{2} \geq c s^{\prime 2}-C\left(\tilde{r}-\tilde{r}^{\prime}\right)^{2} \\
& \geq c s^{\prime 2}-C(\delta)\left(\sin ^{2}\left(\frac{\alpha^{\prime}}{2}\right)+s^{\prime 2}\right) \geq c(\delta) s^{\prime 2}-C(\delta) \sin ^{2}\left(\frac{\alpha^{\prime}}{2}\right) .
\end{aligned}
$$

Then

$$
\left|A^{\prime}\right| \geq c\left(\sin ^{2}\left(\frac{\alpha^{\prime}}{2}\right)+b^{2} s^{\prime 2}\right)
$$

In order to show the second statement we notice that

$$
\begin{aligned}
& \left|\log \left(A^{\prime}\right)\right| \leq|\log (B)| \quad \text { if } \quad 0 \leq B \leq A^{\prime} \leq 1 \\
& \left|\log \left(A^{\prime}\right)\right| \leq|\log (B)| \quad \text { if } \quad 1 \leq A^{\prime} \leq B
\end{aligned}
$$

Since

$$
\begin{aligned}
& \left|A^{\prime}\right| \leq C \\
& \left|A^{\prime}\right| \geq c\left(\sin ^{2}\left(\frac{\alpha^{\prime}}{2}\right)+b^{2} s^{\prime 2}\right)
\end{aligned}
$$

we have that

$$
\left|\log \left(A^{\prime}\right)\right| \leq \log \left(\sin ^{2}\left(\frac{\alpha^{\prime}}{2}\right)^{2}+b^{2} s^{\prime 2}\right)+C .
$$

Just a computation shows that

$$
\partial A^{\prime}=4 b\left(\partial u+\partial u^{\prime}\right) \sin ^{2}\left(\frac{\alpha^{\prime}}{2}\right)+b^{2}\left(4\left(u^{\prime} \partial u+u \partial u^{\prime}\right) \sin ^{2}\left(\frac{\alpha^{\prime}}{2}\right)+2\left(u-u^{\prime}\right)\left(\partial u-\partial u^{\prime}\right)\right) .
$$

Taking modulus we have that

$$
\begin{aligned}
\left|\partial A^{\prime}\right| & \leq C(\delta) b \sin ^{2}\left(\frac{\alpha^{\prime}}{2}\right)+C(\delta) a^{2} \sin ^{2}\left(\frac{\alpha^{\prime}}{2}\right)+C(\delta) b^{2}\left(\sin ^{2}\left(\frac{\alpha^{\prime}}{2}\right)+s^{2}\right) \\
& \leq C b\left(\sin ^{2}\left(\frac{\alpha^{\prime}}{2}\right)+b s^{\prime 2}\right) .
\end{aligned}
$$


Taking two derivatives on $A$ yields

$$
\begin{aligned}
\partial^{2} A^{\prime}= & 4 b \partial^{2}\left(u+u^{\prime}\right) \sin ^{2}\left(\frac{\alpha^{\prime}}{2}\right) \\
& +b^{2}\left(4 \partial^{2}\left(u u^{\prime}\right) \sin ^{2}\left(\frac{\alpha^{\prime}}{2}\right)+2\left(u-u^{\prime}\right) \partial^{2}\left(u-u^{\prime}\right)+2\left(\partial u-\partial u^{\prime}\right)^{2}\right)
\end{aligned}
$$

Taking modulus we obtain

$$
\left|\partial^{2} A^{\prime}\right| \leq C(\delta) b \sin ^{2}\left(\frac{\alpha^{\prime}}{2}\right)+C(\delta) b^{2} \sin ^{2}\left(\frac{\alpha^{\prime}}{2}\right)+C(\delta) b^{2}\left(\left|\sin \left(\frac{\alpha^{\prime}}{2}\right)\right|+\left|s^{\prime}\right|\right)+C(\delta) b^{2}\left(\sin ^{2}\left(\frac{\alpha^{\prime}}{2}\right)+s^{\prime 2}\right) .
$$

Lemma B.2 The following estimate holds for $b>0$,

$$
\int_{-\pi}^{\pi} \int_{-1}^{1} \frac{\left|\sin \left(\frac{\alpha}{2}\right)\right|^{m}|s|^{k}}{\left(\sin ^{2}\left(\frac{\alpha}{2}\right)+b^{2} s^{2}\right)^{l}} d \alpha d s \leq\left\{\begin{array}{cl}
C b^{m+1-2 l} & m<2 l-1 \\
C \log \left(\frac{1}{b}\right) & m=2 l-1 \\
C & m>2 l-1
\end{array},\right.
$$

with $l \geq 1, k, m \geq 0$ and $k+m+1-2 l \geq 0$.

Proof: First we notice that

$$
I \equiv \int_{-\pi}^{\pi} \int_{-1}^{1} \frac{\left|\sin \left(\frac{\alpha}{2}\right)\right|^{m}|s|^{k}}{\left(\sin ^{2}\left(\frac{\alpha}{2}\right)+b^{2} s^{2}\right)^{l}} d \alpha d s=C \int_{0}^{\frac{\pi}{2}} \int_{0}^{1} \frac{\sin (\alpha)^{m} s^{k}}{\left(\sin ^{2}(\alpha)+b^{2} s^{2}\right)^{l}} d \alpha d s
$$

and using that $c \alpha \leq \sin (\alpha) \leq \alpha$ in $[0, \pi / 2]$, we have that

$$
I \leq C \int_{0}^{\frac{\pi}{2}} \int_{0}^{1} \frac{\alpha^{m} s^{k}}{\left(\alpha^{2}+b^{2} s^{2}\right)^{l}} d \alpha d s=\frac{C}{b^{k+1}} \int_{0}^{\frac{\pi}{2}} \int_{0}^{b} \frac{\alpha^{m} s^{k}}{\left(\alpha^{2}+s^{2}\right)^{l}} d \alpha d s .
$$

Making the change of variable

$$
\begin{gathered}
s=r \cos (\theta) \\
\alpha=r \sin (\theta)
\end{gathered}
$$

yields

$$
I \leq \frac{C}{b^{k+1}}\left(\int_{0}^{\arctan \left(\frac{\pi}{2 b}\right)} \int_{0}^{\frac{b}{\cos (\theta)}}+\int_{\arctan \left(\frac{\pi}{2 b}\right)}^{\frac{\pi}{2}} \int_{0}^{\frac{\pi}{2 \sin (\theta)}}\right) r^{k+m+1-2 l} \sin ^{m}(\theta) \cos ^{k}(\theta) d r d \theta .
$$

On one hand

$$
\begin{aligned}
& \int_{0}^{\arctan \left(\frac{\pi}{2 b}\right)} \int_{0}^{\frac{b}{\cos (\theta)}} r^{k+m+1-2 l} \sin ^{m}(\theta) \cos ^{k}(\theta) d r d \theta \\
& =\frac{b^{k+m+2-2 l}}{k+m+2-2 l} \int_{0}^{\arctan \left(\frac{\pi}{2 b}\right)} \tan ^{m}(\theta) \cos ^{2 l-2}(\theta) d \theta=C b^{k+m+2-2 l} \int_{0}^{\arctan \left(\frac{\pi}{2 b}\right)} \frac{\tan ^{m}(\theta)}{\left(1+\tan ^{2}(\theta)\right)^{l-1}} d \theta \\
& =C b^{k+m+2-2 l} \int_{0}^{\frac{1}{b}} \frac{y^{m}}{\left(1+y^{2}\right)^{l-1}\left(1+y^{2}\right)} d y \leq b^{k+m+2-2 l}\left(1+\int_{1}^{\frac{\pi}{2 b}} y^{m-2 l} d y\right) .
\end{aligned}
$$


Thus

$$
\begin{aligned}
& \int_{0}^{\arctan \left(\frac{\pi}{2 b}\right)} \int_{0}^{\frac{b}{\cos (\theta)}} r^{k+m+1-2 l} \sin ^{m}(\theta) \cos ^{k}(\theta) d r d \theta \\
& \leq\left\{\begin{array}{cc}
C b^{k+m+2-2 l}\left(1+\log \left(\frac{\pi}{2 b}\right)\right) & \text { if } m=2 l-1 \\
b^{k+m+2-2 l}\left(1+\frac{1}{m-2 l+1}\left(\left(\frac{\pi}{2 b}\right)^{m-2 l+1}-1\right)\right) & \text { if } m \neq 2 l-1
\end{array}\right. \\
& \leq\left\{\begin{array}{cc}
\left.C b^{k+1} \log \left(\frac{\pi}{2 b}\right)\right) & \text { if } m=2 l-1 \\
C b^{k+1} & \text { if } m>2 l-1 \\
C b^{k+m+2-2 l} & \text { if } m<2 l-1 .
\end{array}\right.
\end{aligned}
$$

On the other hand

$$
\begin{aligned}
& \int_{\arctan \left(\frac{\pi}{2 b}\right)}^{\frac{\pi}{2}} \int_{0}^{\frac{\pi}{2 \sin (\theta)}} r^{k+m+1-2 l} \sin ^{m}(\theta) \cos ^{k}(\theta) d r d \theta \\
& =C \int_{\arctan \left(\frac{\pi}{2 b}\right)}^{\frac{\pi}{2}} \cot ^{k}(\theta) \sin ^{2 l-2}(\theta) d \theta \leq C \int_{\arctan \left(\frac{\pi}{2 a}\right)}^{\frac{\pi}{2}} \cot ^{k}(\theta) d \theta \\
& =C \int_{\frac{\pi}{2 b}}^{\infty} \frac{1}{y^{k}} \frac{1}{1+y^{2}} d y \leq C \int_{\frac{\pi}{2 b}}^{\infty} y^{-k-2} d y=C b^{k+1}
\end{aligned}
$$

Lemma B.3 The following estimate holds for $b>0$

$$
\int_{-\pi}^{\pi} \int_{-1}^{1}\left|\log \left(\sin ^{2}\left(\frac{\alpha^{\prime}}{2}\right)+b^{2} s^{\prime 2}\right)\right| d \alpha^{\prime} d s^{\prime} \leq C
$$

Proof: We just have to notice that

$$
\left|\log \left(\sin ^{2}\left(\frac{\alpha^{\prime}}{2}\right)+b^{2} s^{\prime 2}\right)\right| \leq\left|\log \left(\sin ^{2}\left(\frac{\alpha^{\prime}}{2}\right)\right)\right|+C .
$$

Next we will use lemmas B.1 B.2 and B.3 to prove the following

Lemma B.4 Let $f(\alpha, s) \in L^{2}(\Omega)$ with $\operatorname{supp}(f) \subset \mathbb{T} \times[-1,1]$ and $b>0$. Then

$$
\left\|\int_{-\infty}^{\infty} \int_{-\pi}^{\pi} \log \left(A^{\prime}\right) f^{\prime} d \alpha^{\prime} d s^{\prime}\right\|_{L^{2}} \leq C\|f\|_{L^{2}}
$$

2.

$$
\left\|\int_{-\infty}^{\infty} \int_{-\pi}^{\pi} \frac{\partial A^{\prime}}{A^{\prime}} f^{\prime} d \alpha^{\prime} d s^{\prime}\right\|_{L^{2}} \leq C b\|f\|_{L^{2}}
$$

3.

$$
\left\|\int_{-\infty}^{\infty} \int_{-\pi}^{\pi}\left(\frac{\partial^{2} A^{\prime}}{A^{\prime}}-\frac{\left(\partial A^{\prime}\right)^{2}}{A^{2}}\right) f^{\prime} d \alpha^{\prime} d s^{\prime}\right\|_{L^{2}} \leq C b\|f\|_{L^{2}} .
$$

4.

$$
\left\|\int_{-\infty}^{\infty} \int_{-\pi}^{\pi}\left(\partial^{3} \log \left(A^{\prime}\right)-\frac{\partial^{3} A^{\prime}}{A^{\prime}}\right) f^{\prime} d \alpha^{\prime} d s^{\prime}\right\|_{L^{2}} \leq C b\|f\|_{L^{2}} .
$$


where we remark that $C$ does not depend on $b$.

Proof: To prove 1 we first use Lemma B.1-2, then Minkowski inequality and finally Lemma B.3 to obtain that

$$
\left\|\int_{-\infty}^{\infty} \int_{-\pi}^{\pi} \log \left(A^{\prime}\right) f^{\prime} d \alpha^{\prime} d s^{\prime}\right\|_{L^{2}} \leq C\|f\|_{L^{2}} \int_{-\pi}^{\pi} \int_{-2}^{2}\left(\left|\log \left(\sin ^{2}\left(\frac{\alpha^{\prime}}{2}\right)+b^{2} s^{\prime 2}\right)\right|+C\right) d \alpha^{\prime} d s^{\prime} \leq C .
$$

To prove 2 we first use Lemmas B.1-1 and B.1-3, then Minkowski inequality and finally Lemma B.2 to get

$$
\left\|\int_{-\infty}^{\infty} \int_{-\pi}^{\pi} \frac{\partial A^{\prime}}{A^{\prime}} f^{\prime} d \alpha^{\prime} d s^{\prime}\right\|_{L^{2}} \leq C\|f\|_{L^{2}} \int_{\pi}^{\pi} \int_{-2}^{2} \frac{b\left(\sin ^{2}\left(\frac{\alpha^{\prime}}{2}\right)+b s^{2}\right)}{\sin ^{2}\left(\frac{\alpha^{\prime}}{2}\right)+b^{2} s^{\prime 2}} d \alpha^{\prime} d s^{\prime} \leq C\|f\|_{L^{2}} .
$$

To prove 3 we first use Lemmas B.1-1, B.1-3 and B.1-4 to obtain that

$$
\begin{aligned}
& \left|\frac{\partial^{2} A^{\prime}}{A^{\prime}}\right| \leq C b \frac{\sin ^{2}\left(\frac{\alpha^{\prime}}{2}\right)+b\left(\left|\sin \left(\frac{\alpha^{\prime}}{2}\right)\right|+\left|s^{\prime}\right|+s^{2}\right)}{\sin ^{2}\left(\frac{\alpha^{\prime}}{2}\right)+b^{2} s^{\prime 2}} \\
& \left|\frac{\left(\partial A^{\prime}\right)^{2}}{A^{\prime 2}}\right| \leq C b^{2} \frac{\sin ^{4}\left(\frac{\alpha^{\prime}}{2}\right)+b^{2} s^{\prime 4}}{\left(\sin ^{2}\left(\frac{\alpha^{\prime}}{2}\right)+b^{2} s^{\prime 2}\right)^{2}} .
\end{aligned}
$$

Then we can use Minkowski inequality and Lemma B.2 to yield 3

Finally we prove 4 First we notice that

$$
\partial^{3} \log \left(A^{\prime}\right)-\frac{\partial^{3} A^{\prime}}{A^{\prime}}=-3 \frac{\partial A^{\prime} \partial^{2} A^{\prime}}{A^{\prime 2}}-\frac{\left(\partial A^{\prime}\right)^{3}}{A^{\prime 3}}
$$

and that by lemmas B.1-1, B.1-3, B.1-4, we have that

$$
\begin{aligned}
& \left|\frac{\partial A^{\prime} \partial^{2} A^{\prime}}{A^{\prime 2}}\right| \\
& \leq C b^{2} \frac{\sin ^{4}\left(\frac{\alpha^{\prime}}{2}\right)+b\left|\sin \left(\frac{\alpha^{\prime}}{2}\right)\right|^{3}+b \sin ^{2}\left(\frac{\alpha^{\prime}}{2}\right)\left(\left|s^{\prime}\right|+s^{\prime 2}\right)+b \sin ^{2}\left(\frac{\alpha^{\prime}}{2}\right) s^{\prime 2}+b^{2} s^{\prime 2}\left|\sin \left(\frac{\alpha^{\prime}}{2}\right)\right|+b^{2} s^{\prime 2}\left(\left|s^{\prime}\right|+s^{\prime 2}\right)}{\left(\sin ^{2}\left(\frac{\alpha^{\prime}}{2}\right)+b^{2} s^{\prime 2}\right)^{2}} \\
& +\left|\frac{\left(\partial A^{\prime}\right)^{3}}{A^{\prime 3}}\right| \leq C b^{3} \frac{\sin ^{6}\left(\frac{\alpha^{\prime}}{2}\right)+b^{3} s^{\prime 6}}{\left(\sin ^{2}\left(\frac{\alpha^{\prime}}{2}\right)+b^{2} s^{\prime 2}\right)^{3}} .
\end{aligned}
$$

Thus we can apply Minkowski inequality and lemma B.2 to prove 4

Lemma B.5 Let $f(\alpha, s) \in L^{\infty}(\Omega)$ with $\operatorname{supp}(f) \subset \mathbb{T} \times[-1,1]$ and $b>0$. Then

$$
\left\|\int_{-\infty}^{\infty} \int_{-\pi}^{\pi} \frac{\partial^{3} A^{\prime}}{A^{\prime}} f^{\prime} d \alpha^{\prime} d s^{\prime}\right\|_{L^{2}} \leq C b\|f\|_{L^{\infty}}
$$

Proof: Taking three derivatives on $A^{\prime}$ yields

$$
\partial^{3} A^{\prime}=4 b \partial^{3}\left(u+u^{\prime}\right) \sin ^{2}\left(\frac{\alpha^{\prime}}{2}\right)+
$$




$$
+b^{2}\left(4 \partial^{3}\left(u u^{\prime}\right) \sin ^{2}\left(\frac{\alpha^{\prime}}{2}\right)+6 \partial^{2}\left(u-u^{\prime}\right) \partial\left(u-u^{\prime}\right)+\left(u-u^{\prime}\right)\left(\partial^{3} u-\partial^{3} u^{\prime}\right)\right)
$$

Then

$$
\begin{aligned}
\left|\frac{\partial^{3} A^{\prime}}{A^{\prime}}\right| \leq & C b \frac{\sin ^{2}\left(\frac{\alpha^{\prime}}{2}\right)}{\sin ^{2}\left(\frac{\alpha^{\prime}}{2}\right)+b^{2} s^{\prime 2}}\left(\partial^{3} u+\partial^{3} u^{\prime}\right)+C b^{2} \frac{\sin ^{2}\left(\frac{\alpha^{\prime}}{2}\right)}{\sin ^{2}\left(\frac{\alpha^{\prime}}{2}\right)+b^{2} s^{\prime 2}} \partial^{3}\left(u u^{\prime}\right) \\
& +C b^{2} \frac{\left|\sin \left(\frac{\alpha^{\prime}}{2}\right)\right|+\left|s^{\prime}\right|}{\sin ^{2}\left(\frac{\alpha^{\prime}}{2}\right)+b^{2} s^{\prime 2}}+C b^{2} \frac{\left|\sin \left(\frac{\alpha^{\prime}}{2}\right)\right|+\left|s^{\prime}\right|}{\sin ^{2}\left(\frac{\alpha^{\prime}}{2}\right)+b^{2} s^{\prime 2}}\left|\partial^{3} u-\partial^{3} u^{\prime}\right| .
\end{aligned}
$$

Then by applying lemma B.2 we achieve the conclusion on the lemma.

Lemma B.6 The following estimate holds:

$$
\left|\log \left(\frac{A^{\prime}}{A^{\prime}[0]}\right)\right| \leq\left|\log \left(\frac{(4-C b) \sin ^{2}\left(\frac{\alpha^{\prime}}{2}\right)+c b^{2} s^{\prime 2}}{4 \sin ^{2}\left(\frac{\alpha^{\prime}}{2}\right)}\right)\right|+\left|\log \left(\frac{\left(4+C b+C b^{2}\right) \sin ^{2}\left(\frac{\alpha^{\prime}}{2}\right)+C b^{2} s^{\prime 2}}{4 \sin ^{2}\left(\frac{\alpha^{\prime}}{2}\right)}\right)\right|
$$

Proof: We notice as in Lemma B.1-2 that

$$
\left|\log \left(\frac{A^{\prime}}{A^{\prime}[0]}\right)\right| \leq\left|\log \left(\frac{\left(4-C(\delta) b-C(\delta) b^{2}\right) \sin ^{2}\left(\frac{\alpha^{\prime}}{2}\right)+c b^{2} s^{\prime 2}}{4 \sin ^{2}\left(\frac{\alpha^{\prime}}{2}\right)}\right)\right|+\left|\log \left(\frac{\left(4+C b+C b^{2}\right) \sin ^{2}\left(\frac{\alpha^{\prime}}{2}\right)+C b^{2} s^{\prime 2}}{4 \sin ^{2}\left(\frac{\alpha^{\prime}}{2}\right)}\right)\right|
$$

since

$$
\left|A^{\prime}\right| \geq\left(1-C(\delta) b-C(\delta) b^{2}\right) \sin ^{2}\left(\frac{\alpha^{\prime}}{2}\right)+c b^{2} s^{2}
$$

as we checked in the proof of lemma B.1 1 and

$$
A^{\prime} \leq C\left(\left(1+b+b^{2}\right) \sin ^{2}\left(\frac{\alpha^{\prime}}{2}\right)+b^{2} s^{\prime 2}\right) .
$$

Lemma B.7 The following inequalities hold for $b>0$.

$$
\begin{aligned}
& \int_{-\pi}^{\pi} \int_{-2}^{2}\left|\log \left(\frac{(1-b) \sin ^{2}\left(\frac{\alpha^{\prime}}{2}\right)+b^{2} s^{2}}{\sin ^{2}\left(\frac{a l^{\prime}}{2}\right)}\right)\right| d \alpha d s^{\prime} \leq C b \\
& \int_{-\pi}^{\pi} \int_{-2}^{2}\left|\log \left(\frac{(1+b) \sin ^{2}\left(\frac{\alpha^{\prime}}{2}\right)+b^{2} s^{\prime 2}}{\sin ^{2}\left(\frac{a l^{\prime}}{2}\right)}\right)\right| d \alpha d s^{\prime} \leq C b
\end{aligned}
$$

Proof: We notice that

$$
\left|\log \left(1-b+\frac{b^{2} s^{\prime 2}}{\sin ^{2}\left(\frac{\alpha^{\prime}}{2}\right)}\right)\right| \leq|\log (1-b)|+\left|\log \left(1+\frac{b^{2} s^{2}}{\sin ^{2}\left(\frac{\alpha^{\prime}}{2}\right)}\right)\right| .
$$


Then the estimate follows from

$$
\int_{-\pi}^{\pi} \log \left(1+\frac{b^{2}}{\sin ^{2}\left(\frac{\alpha^{\prime}}{2}\right)}\right) d \alpha^{\prime}=4 \pi \operatorname{arcsinh}(b) .
$$

In addition, the second inequality follows from

$$
\int_{-\pi}^{\pi} \log \left(1+b+\frac{b^{2}}{\sin ^{2}\left(\frac{\alpha^{\prime}}{2}\right)}\right) d \alpha^{\prime}=4 \pi \log \left(b+\sqrt{1+b+b^{2}}\right) .
$$

Lemma B.8 Let $f(\alpha, s) \in L^{2}$ with $\operatorname{supp}(f) \in \mathbb{T} \times[-1,1]$. Then

$$
\left\|\int_{-\infty}^{\infty} \int_{-\pi}^{\pi} \frac{1}{b} \log \left(\frac{A^{\prime}}{A^{\prime}[0]}\right) f^{\prime} d \alpha^{\prime} d s^{\prime}\right\|_{L^{2}} \leq C\|f\|_{L^{2}} .
$$

Proof: This lemma is a consequence of Lemmas B.6 and B.7 and Minkowski inequality.

The following lemmas will be used to show the differentiability of the functional $\mathcal{G}$ and the continuity of its derivative.

Here we recall that

$$
\partial_{a} A^{\prime}=8 a\left(u+u^{\prime}\right) \sin ^{2}\left(\frac{\alpha^{\prime}}{2}\right)+4 a^{3}\left(u u^{\prime} \sin ^{2}\left(\frac{\alpha^{\prime}}{2}\right)+\left(u-u^{\prime}\right)^{2}\right)
$$

Lemma B.9 The following estimates hold:

1.

$$
\left|\partial_{a} A^{\prime}\right| \leq C|a|\left(\left(1+a^{2}\right) \sin ^{2}\left(\frac{\alpha^{\prime}}{2}\right)+a^{2} s^{\prime 2}\right) .
$$

2.

$$
\left|\partial \partial_{a} A^{\prime}\right| \leq C|a|\left(\left(1+a^{2}\right) \sin ^{2}\left(\frac{\alpha^{\prime}}{2}\right)+a^{2} s^{\prime 2}\right) .
$$

3.

$$
\left|\partial^{2} \partial_{a} A^{\prime}\right| \leq C|a|\left(\left(1+a^{2}\right) \sin ^{2}\left(\frac{\alpha^{\prime}}{2}\right)+a^{2}\left|\sin \left(\frac{\alpha^{\prime}}{2}\right)\right|+a^{2}\left(\left|s^{\prime}\right|+s^{\prime 2}\right)\right) .
$$

Proof: The proof of this lemma follows similar steps as the proof of Lemma B.1.

Lemma B.10 Let $f(\alpha, s) \in L^{2}(\Omega)$ with $\operatorname{supp}(f) \subset \mathbb{T} \times[-1,1]$ and $b>0$. Then

1.

$$
\left\|\int_{-\infty}^{\infty} \int_{-\pi}^{\pi} \frac{\partial_{a} A^{\prime}}{A^{\prime}} f^{\prime} d \alpha^{\prime} d s^{\prime}\right\|_{L^{2}} \leq C|a|\|f\|_{L^{2}}
$$

2.

$$
\left\|\int_{-\infty}^{\infty} \int_{-\pi}^{\pi} \partial \frac{\partial_{a} A^{\prime}}{A^{\prime}} f^{\prime} d \alpha^{\prime} d s^{\prime}\right\|_{L^{2}} \leq C|a|\|f\|_{L^{2}}
$$

3.

$$
\left\|\int_{-\infty}^{\infty} \int_{-\pi}^{\pi} \partial^{2} \frac{\partial_{a} A^{\prime}}{A^{\prime}} f^{\prime} d \alpha^{\prime} d s^{\prime}\right\|_{L^{2}} \leq C|a|\|f\|_{L^{2}}
$$


4.

$$
\left\|\int_{-\infty}^{\infty} \int_{-\pi}^{\pi}\left(\partial^{3}\left(\frac{\partial_{a} A^{\prime}}{A^{\prime}}\right)-\frac{\partial^{3} \partial_{a} A^{\prime}}{A^{\prime}}+\frac{\partial_{a} A^{\prime} \partial^{3} A^{\prime}}{A^{\prime 2}}\right) f^{\prime} d \alpha^{\prime} d s^{\prime}\right\|_{L^{2}} \leq C|a|\|f\|_{L^{2}}
$$

Proof: In order to prove 1 we notice that by Lemmas B.1 1 and B.9-1

$$
\left|\frac{\partial_{a} A^{\prime}}{A^{\prime}}\right| \leq C|a| \frac{\sin ^{2}\left(\frac{\alpha^{\prime}}{2}\right)+a^{2} s^{\prime 2}}{\sin ^{2}\left(\frac{\alpha^{\prime}}{2}\right)+a^{4} s^{\prime 2}} \leq C|a|+|a|^{3} \frac{s^{\prime 2}}{\sin ^{2}\left(\frac{\alpha^{\prime}}{2}\right)+a^{4} s^{\prime 2}}
$$

Therefore we obtain the first inequality in lemma B.9 by applying Minkowski's inequality and Lemma B.2

To obtain 2 we proceed as follows

$$
\partial \frac{\partial_{a} A^{\prime}}{A^{\prime}}=\frac{\partial \partial_{a} A^{\prime}}{A^{\prime}}-\frac{\partial_{a} A^{\prime} \partial A^{\prime}}{A^{\prime 2}}
$$

Then, by applying Lemmas B.1 1, B.1 3, B.9.1 and B.9.2, we have that

$$
\left|\partial \frac{\partial_{a} A^{\prime}}{A^{\prime}}\right| \leq C|a| \frac{\sin ^{2}\left(\frac{\alpha^{\prime}}{2}\right)+a^{2} s^{\prime 2}}{\sin ^{2}\left(\frac{\alpha^{\prime}}{2}\right)+a^{4} s^{\prime 2}}+C|a|^{3} \frac{\sin ^{4}\left(\frac{\alpha^{\prime}}{2}\right)+a^{4} s^{\prime 4}}{\left(\sin ^{2}\left(\frac{\alpha^{\prime}}{2}\right)+a^{4} s^{\prime 2}\right)^{2}}
$$

Then we can use again Minkowski's inequality and Lemma B.2 to yield 2.

Taking one more derivative yields

$$
\partial^{2} \frac{\partial_{a} A^{\prime}}{A^{\prime}}=\frac{\partial^{2} \partial_{a} A^{\prime}}{A^{\prime}}-2 \frac{\partial \partial_{a} A^{\prime} \partial A^{\prime}}{A^{\prime 2}}-\frac{\partial_{a} A^{\prime} \partial^{2} A^{\prime}}{A^{\prime 2}}+2 \frac{\partial_{a} A^{\prime}\left(\partial A^{\prime}\right)^{2}}{A^{\prime 3}} .
$$

By using Lemmas B.1 1, B.1 3 and $\overline{B .9}+3$ we can estimate

$$
\left|\frac{\partial \partial_{a} A^{\prime} \partial A^{\prime}}{A^{\prime 2}}\right| \leq C|a|^{3} \frac{\sin ^{4}\left(\frac{\alpha^{\prime}}{2}\right)+a^{4} s^{\prime 4}}{\left(\sin ^{2}\left(\frac{\alpha^{\prime}}{2}\right)+a^{4} s^{\prime 2}\right)^{2}} .
$$

By using Lemmas B.1 1, B.9 1 and B.1-3 we can estimate

$$
\left|\frac{\partial_{a} A^{\prime}\left(\partial A^{\prime}\right)^{2}}{A^{\prime 3}}\right| \leq C|a|^{5} \frac{\left(\sin ^{2}\left(\frac{\alpha^{\prime}}{2}\right)+a^{2} s^{\prime 2}\right)^{3}}{\left(\sin ^{2}\left(\frac{\alpha^{\prime}}{2}\right)+a^{4} s^{\prime 2}\right)^{3}} \leq C|a|^{7} \frac{\sin ^{6}\left(\alpha^{\prime}\right)+a^{6} s^{\prime 6}}{\left(\sin ^{2}\left(\frac{\alpha^{\prime}}{2}\right)+a^{4} s^{\prime 2}\right)^{3}} .
$$

By using Lemmas B.1 1 and B.9. 3 we can estimate

$$
\left|\frac{\partial^{2} \partial_{a} A^{\prime}}{A^{\prime}}\right| \leq C|a| \frac{\left(\sin ^{2}\left(\frac{\alpha^{\prime}}{2}\right)+a^{2}\left|\sin \left(\frac{\alpha^{\prime}}{2}\right)\right|+a^{2}\left(\left|s^{\prime}\right|+s^{\prime 2}\right)\right)}{\sin ^{2}\left(\frac{\alpha^{\prime}}{2}\right)+a^{4} s^{\prime 2}} .
$$

Finally, by using Lemmas B.1 1, B.1-3 and B.9.1 we have that

$$
\begin{aligned}
& \left|\frac{\partial_{a} \partial^{2} A^{\prime}}{A^{\prime 2}}\right| \leq C|a|^{3} \frac{\left(\sin ^{2}\left(\frac{\alpha^{\prime}}{2}\right)+a^{2} s^{\prime 2}\right)\left(\sin ^{2}\left(\frac{\alpha^{\prime}}{2}\right)+a^{2}\left|\sin \left(\frac{\alpha^{\prime}}{2}\right)\right|+a^{2}\left(\left|s^{\prime}\right|+s^{\prime 2}\right)\right)}{\left(\sin ^{2}\left(\frac{\alpha^{\prime}}{2}\right)+a^{4} s^{\prime 2}\right)^{2}} \\
& \leq C|a|^{3} \frac{\sin ^{4}\left(\frac{\alpha^{\prime}}{2}\right)+a^{2}\left|\sin \left(\frac{\alpha^{\prime}}{2}\right)\right|^{3}+a^{2} \sin ^{2}\left(\frac{\alpha^{\prime}}{2}\right)\left(\left|s^{\prime}\right|+s^{\prime 2}\right)+a^{2} \sin ^{2}\left(\frac{\alpha^{\prime}}{2}\right) s^{\prime 2}+a^{4}\left|\sin \left(\frac{\alpha^{\prime}}{2}\right)\right| s^{\prime 2}+a^{4} s^{\prime 2}\left(\left|s^{\prime}\right|+s^{\prime 2}\right)}{\left(\sin ^{2}\left(\frac{\alpha^{\prime}}{2}\right)+a^{4} s^{\prime 2}\right)^{2}}
\end{aligned}
$$


Then by applying Minkowski's inequality and Lemma B.2 we achieve 3 .

In order to prove 4 , we start taking 3 derivatives:

$$
\begin{aligned}
& \partial^{3} \frac{\partial_{a} A^{\prime}}{A^{\prime}}-\frac{\partial^{3} \partial_{a} A^{\prime}}{A^{\prime}}+\frac{\partial_{a} A^{\prime} \partial^{3} A^{\prime}}{A^{\prime 2}}=-3 \frac{\partial^{2} \partial_{a} A^{\prime} \partial A^{\prime}}{A^{\prime 2}}-3 \frac{\partial \partial_{a} A^{\prime} \partial^{2} A^{\prime}}{A^{\prime 2}} \\
& +6 \frac{\partial \partial_{a} A^{\prime}\left(\partial A^{\prime}\right)^{2}}{A^{\prime 3}}+6 \frac{\partial_{a} A^{\prime} \partial^{2} A^{\prime} \partial A^{\prime}}{A^{\prime 3}}-6 \frac{\partial_{a} A^{\prime}\left(\partial A^{\prime}\right)^{3}}{A^{\prime 4}}
\end{aligned}
$$

By using Lemmas B.1 1, B.1-3 and B.9.3 we can estimate

$$
\begin{aligned}
& \left|\frac{\partial^{2} \partial_{a} A^{\prime} \partial A^{\prime}}{A^{\prime 2}}\right| \\
& \leq C|a|^{3} \frac{\left(\sin ^{2}\left(\frac{\alpha^{\prime}}{2}\right)+a^{2} s^{\prime 2}\right)\left(\sin ^{2}\left(\frac{\alpha^{\prime}}{2}\right)+a^{2}\left|\sin \left(\frac{\alpha^{\prime}}{2}\right)\right|+a^{2}\left(\left|s^{\prime}\right|+s^{\prime 2}\right)\right)}{\left(\sin ^{2}\left(\frac{\alpha^{\prime}}{2}\right)+a^{4} s^{\prime 2}\right)^{2}} \\
& \leq C|a|^{3} \frac{\sin ^{4}\left(\frac{\alpha^{\prime}}{2}\right)+a^{2}\left|\sin \left(\frac{\alpha^{\prime}}{2}\right)\right|^{3}+a^{2} \sin ^{2}\left(\frac{\alpha^{\prime}}{2}\right)\left(\left|s^{\prime}\right|+s^{\prime 2}\right)+a^{2} s^{\prime 2} \sin ^{2}\left(\frac{\alpha^{\prime}}{2}\right)+a^{4} s^{\prime 2}\left|\sin \left(\frac{\alpha^{\prime}}{2}\right)\right|+a^{4} s^{\prime 2}\left(\left|s^{\prime}\right|+s^{\prime 2}\right)}{\left(\sin ^{2}\left(\frac{\alpha^{\prime}}{2}\right)+a^{4} s^{\prime 2}\right)^{2}}
\end{aligned}
$$

By using Lemmas B.1 1, B.1 4 and B.9-2 we can estimate

$$
\left|\frac{\partial \partial_{a} A^{\prime} \partial^{2} A^{\prime}}{A^{\prime 2}}\right| \leq C|a|^{3} \frac{\left(\sin ^{2}\left(\frac{\alpha^{\prime}}{2}\right)+a^{2} s^{\prime 2}\right)\left(\sin ^{2}\left(\frac{\alpha^{\prime}}{2}\right)+a^{2}\left|\sin \left(\frac{\alpha^{\prime}}{2}\right)\right|+a^{2}\left(\left|s^{\prime}\right|+s^{\prime 2}\right)\right)}{\left(\sin ^{2}\left(\frac{\alpha^{\prime}}{2}\right)+a^{4} s^{\prime 2}\right)^{2}},
$$

and this term is done as the previous one.

By using Lemmas B.1 1, B.1 3 and B.9-2 we can estimate

$$
\begin{aligned}
\left|\frac{\partial \partial_{a} A^{\prime}\left(\partial A^{\prime}\right)^{2}}{A^{\prime 3}}\right| & \leq C|a|^{5} \frac{\left(\sin ^{2}\left(\frac{\alpha^{\prime}}{2}\right)+a^{2} s^{\prime 2}\right)^{3}}{\left(\sin ^{2}\left(\frac{\alpha^{\prime}}{2}\right)+a^{4} s^{\prime 2}\right)^{3}} \\
& \leq C|a|^{5} \frac{\left(\sin ^{6}\left(\frac{\alpha^{\prime}}{2}\right)+a^{6} s^{\prime 6}\right)}{\left(\sin ^{2}\left(\frac{\alpha^{\prime}}{2}\right)+a^{4} s^{\prime 2}\right)^{3}}
\end{aligned}
$$

By using Lemmas B.1 1, B.4 3, B.1.4 and B.9.1 we can estimate

$$
\begin{aligned}
& \left|\frac{\partial_{a} A^{\prime} \partial^{2} A^{\prime} \partial A^{\prime}}{A^{\prime 3}}\right| \\
& \leq C|a|^{5} \frac{\left(\sin ^{2}\left(\frac{\alpha^{\prime}}{2}\right)+a^{2} s^{\prime 2}\right)^{2}\left(\sin ^{2}\left(\frac{\alpha^{\prime}}{2}\right)+a^{2}\left|\sin \left(\frac{\alpha^{\prime}}{2}\right)\right|+a^{2}\left(\left|s^{\prime}\right|+s^{\prime 2}\right)\right)}{\left(\sin ^{2}\left(\frac{\alpha^{\prime}}{2}\right)+a^{4} s^{\prime 2}\right)^{3}} \\
& \leq C|a|^{5} \frac{\left(\sin ^{4}\left(\frac{\alpha^{\prime}}{2}\right)+a^{4} s^{\prime 4}\right)\left(\sin ^{2}\left(\frac{\alpha^{\prime}}{2}\right)+a^{2}\left|\sin \left(\frac{\alpha^{\prime}}{2}\right)\right|+a^{2}\left(\left|s^{\prime}\right|+s^{\prime 2}\right)\right)}{\left(\sin ^{2}\left(\frac{\alpha^{\prime}}{2}\right)+a^{4} s^{\prime 2}\right)^{3}}
\end{aligned}
$$




$$
\leq C|a|^{3} \frac{\sin ^{6}\left(\frac{\alpha^{\prime}}{2}\right)+a^{2}\left|\sin \left(\frac{\alpha^{\prime}}{2}\right)\right|^{5}+a^{2} \sin ^{4}\left(\frac{\alpha^{\prime}}{2}\right)\left(\left|s^{\prime}\right|+s^{\prime 2}\right)+a^{4} s^{\prime 4} \sin ^{2}\left(\frac{\alpha^{\prime}}{2}\right)+a^{6} s^{\prime 4}\left|\sin \left(\frac{\alpha^{\prime}}{2}\right)\right|+a^{6} s^{\prime 4}\left(\left|s^{\prime}\right|+s^{\prime 2}\right)}{\left(\sin ^{2}\left(\frac{\alpha^{\prime}}{2}\right)+a^{4} s^{\prime 2}\right)^{3}}
$$

By using Lemmas B.1 1, B.4 3 and B.9.1 we can estimate

$$
\begin{aligned}
\left|\frac{\partial_{a} A^{\prime}\left(\partial A^{\prime}\right)^{3}}{A^{\prime 4}}\right| & \leq C|a|^{7} \frac{\left(\sin ^{2}\left(\frac{\alpha^{\prime}}{2}\right)+a^{2} s^{\prime 2}\right)^{4}}{\left(\sin ^{2}\left(\frac{\alpha^{\prime}}{2}\right)+a^{4} s^{\prime 2}\right)^{4}} \\
& \leq C|a|^{7} \frac{\left(\sin ^{8}\left(\frac{\alpha^{\prime}}{2}\right)+a^{8} s^{\prime}\right)}{\left(\sin ^{2}\left(\frac{\alpha^{\prime}}{2}\right)+a^{4} s^{\prime 2}\right)^{4}} .
\end{aligned}
$$

Finally, by applying Minkowski's inequality and Lemma B.2 we conclude 4.

Lemma B.11 Let $f(\alpha, s) \in L^{\infty}(\Omega)$ with $\operatorname{supp}(f) \subset \mathbb{T} \times[-1,1]$ and $b>0$. Then:

$$
\left\|\int_{-\infty}^{\infty} \int_{-\pi}^{\pi} \frac{\partial^{3} \partial_{a} A^{\prime}}{A^{\prime}} f^{\prime} d \alpha^{\prime} d s^{\prime}\right\|_{L^{2}} \leq C|a|\|f\|_{L^{\infty}} .
$$

and

$$
\left\|\int_{-\infty}^{\infty} \int_{-\pi}^{\pi} \frac{\partial^{3} A^{\prime} \partial_{a} A^{\prime}}{A^{\prime 2}} f^{\prime} d \alpha^{\prime} d s^{\prime}\right\|_{L^{2}} \leq C|a|\|f\|_{L^{\infty}}
$$

Proof: We start computing

$$
\partial^{3} \partial_{a} A^{\prime}=8 a \partial^{3}\left(u+u^{\prime}\right) \sin ^{2}\left(\frac{\alpha^{\prime}}{2}\right)+4 a^{3}\left(\partial^{3}\left(u u^{\prime}\right) \sin ^{2}\left(\frac{\alpha^{\prime}}{2}\right)+6 \partial\left(u-u^{\prime}\right) \partial^{2}\left(u-u^{\prime}\right)+2\left(u-u^{\prime}\right) \partial^{3}\left(u-u^{\prime}\right)\right) .
$$

Then, by using Lemma B.1 1:

$$
\begin{aligned}
\frac{\left|\partial^{3} \partial_{a} A^{\prime}\right|}{|A|} & \leq C|a| \frac{\sin ^{2}\left(\frac{\alpha^{\prime}}{2}\right)}{\left(\sin ^{2}\left(\frac{\alpha^{\prime}}{2}\right)+a^{4} s^{\prime 2}\right)}\left|\partial^{3}\left(u+u^{\prime}\right)\right|+C|a|^{3} \frac{\sin ^{2}\left(\frac{\alpha^{\prime}}{2}\right)}{\left(\sin ^{2}\left(\frac{\alpha^{\prime}}{2}\right)+a^{4} s^{\prime 2}\right)}\left|\partial^{3}\left(u u^{\prime}\right)\right| \\
& +C|a|^{3} \frac{\left|\sin \left(\frac{\alpha^{\prime}}{2}\right)\right|+\left|s^{\prime}\right|}{\left(\sin ^{2}\left(\frac{\alpha^{\prime}}{2}\right)+a^{4} s^{\prime 2}\right)}+C|a|^{3} \frac{\left|\sin \left(\frac{\alpha^{\prime}}{2}\right)\right|+\left|s^{\prime}\right|}{\left(\sin ^{2}\left(\frac{\alpha^{\prime}}{2}\right)+a^{4} s^{\prime 2}\right)}\left|\partial^{3} u-\partial^{3} u^{\prime}\right|,
\end{aligned}
$$

and we can conclude as in Lemma B.5. This shows the first estimate. In order to prove the second one, we use (B.3) and (B.4).

Lemma B.12 Let $f(\alpha, s) \in L^{2}(\Omega)$ with $\operatorname{supp}(f) \subset \mathbb{T} \times[-1,1]$ and $h \neq 0$. Then

1.

$$
\left\|\int_{-\infty}^{\infty} \int_{-\pi}^{\pi} \frac{1}{h} \log \left(\frac{A^{\prime}[h]}{A^{\prime}[0]}\right) f^{\prime} d \alpha^{\prime} d s^{\prime}\right\|_{L^{2}} \leq C|h|\|f\|_{L^{2}}
$$


2.

$$
\left\|\int_{-\infty}^{\infty} \int_{-\pi}^{\pi} \frac{1}{h} \frac{\partial A^{\prime}[h]}{A^{\prime}[h]} f^{\prime} d \alpha^{\prime} d s^{\prime}\right\|_{L^{2}} \leq C|h|\|f\|_{L^{2}}
$$

3.

$$
\left\|\int_{-\infty}^{\infty} \int_{-\pi}^{\pi} \frac{1}{h}\left(\frac{\partial^{2} A^{\prime}[h]}{A^{\prime}[h]}-\frac{\left(\partial A^{\prime}[h]\right)^{2}}{A^{\prime 2}[h]}\right) f^{\prime} d \alpha^{\prime} d s^{\prime}\right\|_{L^{2}} \leq C|h|\|f\|_{L^{2}}
$$

4.

$$
\left\|\int_{-\infty}^{\infty} \int_{-\pi}^{\pi} \frac{1}{h}\left(\partial^{3} \log \left(A^{\prime}[h]\right)-\frac{\partial^{3} A^{\prime}[h]}{A^{\prime}[h]}\right) f^{\prime} d \alpha^{\prime} d s^{\prime}\right\|_{L^{2}} \leq C \mid h\|\| f \|_{L^{2}}
$$

Proof: The inequalities 2 , 3 and 4 follow directly from lemma B.4 by making $b=h^{2}$. Then it remains to show 1. In order to do it we notice that

$$
\frac{1}{h} \log \left(\frac{A^{\prime}[h]}{A^{\prime}[0]}\right)=h \int_{0}^{1} \frac{4\left(u+u^{\prime}\right) \sin ^{2}\left(\frac{\alpha^{\prime}}{2}\right)+4 h^{2} u u^{\prime} \sin ^{2}\left(\frac{\alpha^{\prime}}{2}\right)+h^{2}\left(u-u^{\prime}\right)^{2}}{4 \sin ^{2}\left(\frac{\alpha}{2}\right)+4 \mu h^{2}\left(u+u^{\prime}\right) \sin ^{2}\left(\frac{\alpha^{\prime}}{2}\right)+4 \mu h^{4} u u^{\prime} \sin ^{2}\left(\frac{\alpha}{2}\right)+\mu h^{4}\left(u-u^{\prime}\right)^{2}} d \mu .
$$

Similar computations that those ones to prove B.1 1 yield

$4 \sin ^{2}\left(\frac{\alpha}{2}\right)+4 \mu h^{2}\left(u+u^{\prime}\right) \sin ^{2}\left(\frac{\alpha^{\prime}}{2}\right)+4 \mu h^{4} u u^{\prime} \sin ^{2}\left(\frac{\alpha}{2}\right)+\mu h^{4}\left(u-u^{\prime}\right)^{2} \geq c\left(\sin ^{2}\left(\frac{\alpha^{\prime}}{2}\right)+\left(\sqrt{\mu} h^{2}\right)^{2} s^{\prime 2}\right)$

And we can estimate

$$
\left|\frac{1}{h} \log \left(\frac{A^{\prime}[h]}{A^{\prime}[0]}\right)\right| \leq C h+C h^{3} \int_{0}^{1} \frac{s^{\prime 2}}{\sin ^{2}\left(\frac{\alpha^{\prime}}{2}\right)+\left(\sqrt{\mu} h^{2}\right)^{2} s^{\prime 2}} d \mu
$$

Then we achieve 1 by using Minkowski's inequality and lemma B.2.

Lemma B.13 Let $f(\alpha, s) \in L^{\infty}(\Omega)$ with $\operatorname{supp}(f) \subset \mathbb{T} \times[-1,1]$ and $b>0$. Then

$$
\left\|\int_{-\infty}^{\infty} \int_{-\pi}^{\pi} \frac{1}{h} \frac{\partial^{3} A^{\prime}}{A^{\prime}} f^{\prime} d \alpha^{\prime} d s^{\prime}\right\|_{L^{2}} \leq C|h|\|f\|_{L^{\infty}} .
$$

Proof: This lemma follows from lemma B.5 by making again $b=h^{2}$.

Lemma B.14 The following estimates hold

1.

$$
\left|\frac{1}{b} \log \left(\frac{A[a]}{A[0]}\right)-\left(u+u^{\prime}\right)\right| \leq C b \int_{0}^{1} \frac{\sin ^{2}\left(\frac{\alpha^{\prime}}{2}\right)+s^{\prime 2}}{\sin ^{2}\left(\frac{\alpha^{\prime}}{2}\right)+b^{2} \mu s^{2}} d \mu
$$

2.

$$
\left|\partial \frac{1}{b} \log \left(\frac{A[a]}{A[0]}\right)-\partial\left(u+u^{\prime}\right)\right| \leq C b \frac{\sin ^{2}\left(\frac{\alpha^{\prime}}{2}\right)+s^{\prime 2}}{\sin ^{2}\left(\frac{\alpha^{\prime}}{2}\right)+b^{2} s^{\prime 2}}
$$


3.

$$
\begin{aligned}
\left|\partial^{2}\left(\frac{1}{b} \log \left(\frac{A[a]}{A[0]}\right)-\left(u+u^{\prime}\right)\right)\right| \leq & C b \frac{\sin ^{2}\left(\frac{\alpha^{\prime}}{2}\right)+s^{\prime 2}+\left|\sin \left(\frac{\alpha^{\prime}}{2}\right)\right|+\left|s^{\prime}\right|}{\sin ^{2}\left(\frac{\alpha^{\prime}}{2}\right)+b^{2} s^{\prime 2}} \\
& +C b \frac{\left(\sin ^{2}\left(\frac{\alpha^{\prime}}{2}\right)+b s^{\prime 2}\right)^{2}}{\left(\sin ^{2}\left(\frac{\alpha^{\prime}}{2}\right)+b^{2} s^{\prime 2}\right)^{2}}
\end{aligned}
$$

4.

$$
\begin{aligned}
& \left|\partial^{3}\left(\frac{1}{b} \log \left(\frac{A[a]}{A[0]}\right)-\left(u+u^{\prime}\right)\right)-\left(\frac{1}{b} \frac{\partial^{3} A[a]}{A[a]}-\partial^{3}\left(u+u^{\prime}\right)\right)\right| \\
& \leq C b \frac{\left(\sin ^{2}\left(\frac{\alpha^{\prime}}{2}\right)+b s^{\prime 2}\right)\left(\sin ^{2}\left(\frac{\alpha^{\prime}}{2}\right)+b\left(\left|\sin \left(\frac{\alpha^{\prime}}{2}\right)+s^{\prime 2}+\right| s^{\prime} \mid\right)\right)}{\left(\sin ^{2}\left(\frac{\alpha^{\prime}}{2}\right)+b^{2} s^{\prime 2}\right)^{2}} \\
& +C b^{2} \frac{\left(\sin ^{2}\left(\frac{\alpha^{\prime}}{2}\right)+b s^{\prime 3}\right)^{3}}{\left(\sin ^{2}\left(\frac{\alpha^{\prime}}{2}\right)+b^{2} s^{\prime 2}\right)^{3}}
\end{aligned}
$$

5.

$$
\begin{aligned}
& \left|\frac{1}{b} \frac{\partial^{3} A[a]}{A[a]}-\partial^{3}\left(u+u^{\prime}\right)\right| \leq C b \frac{\sin ^{2}\left(\frac{\alpha^{\prime}}{2}\right)+b s^{\prime 2}}{\sin ^{2}\left(\frac{\alpha^{\prime}}{2}\right)+b^{2} s^{\prime 2}}\left|\partial^{3}\left(u+u^{\prime}\right)\right| \\
& C b \frac{\sin ^{2}\left(\frac{\alpha^{\prime}}{2}\right)}{\sin ^{2}\left(\frac{\alpha^{\prime}}{2}\right)+b^{2} s^{\prime 2}}\left|\partial^{3}\left(u u^{\prime}\right)\right|+C b \frac{\left|\sin \left(\frac{\alpha^{\prime}}{2}\right)\right|+\left|s^{\prime}\right|}{\sin ^{2}\left(\frac{\alpha^{\prime}}{2}\right)+b^{2} s^{\prime 2}}\left|\partial^{3}\left(u-u^{\prime}\right)\right|+C b \frac{\left|\sin \left(\frac{\alpha^{\prime}}{2}\right)\right|+\left|s^{\prime}\right|}{\sin ^{2}\left(\frac{\alpha^{\prime}}{2}\right)+b^{2} s^{\prime 2}} .
\end{aligned}
$$

Proof:

$$
\begin{aligned}
& \frac{1}{b} \log \left(\frac{A[a]}{A[0]}\right)=\frac{1}{b} \int_{0}^{1} \frac{d}{d \mu} \log \left(4 \sin ^{2}\left(\frac{\alpha^{\prime}}{2}\right)+4 b \mu\left(u+u^{\prime}\right) \sin ^{2}\left(\frac{\alpha^{\prime}}{2}\right)+b^{2} \mu\left(4 u u^{\prime} \sin ^{2}\left(\frac{\alpha^{\prime}}{2}\right)+\left(u-u^{\prime}\right)^{2}\right)\right) d \mu \\
& =\int_{0}^{1} \frac{4\left(u+u^{\prime}\right) \sin ^{2}\left(\frac{\alpha^{\prime}}{2}\right)+b\left(4 u u^{\prime} \sin ^{2}\left(\frac{\alpha^{\prime}}{2}\right)+\left(u-u^{\prime}\right)^{2}\right)}{4 \sin ^{2}\left(\frac{\alpha^{\prime}}{2}\right)+4 b \mu\left(u+u^{\prime}\right) \sin ^{2}\left(\frac{\alpha^{\prime}}{2}\right)+b^{2} \mu\left(4 u u^{\prime} \sin ^{2}\left(\frac{\alpha^{\prime}}{2}\right)+\left(u-u^{\prime}\right)^{2}\right)} d \mu
\end{aligned}
$$

Then

$$
\begin{aligned}
& \frac{1}{b} \log \left(\frac{A[a]}{A[0]}\right)-\left(u+u^{\prime}\right)=b \int_{0}^{1} \frac{4 u u^{\prime} \sin ^{2}\left(\frac{\alpha^{\prime}}{2}\right)+\left(u-u^{\prime}\right)^{2}+4 \mu\left(u+u^{\prime}\right)^{2} \sin ^{2}\left(\frac{\alpha^{\prime}}{2}\right)}{4 \sin ^{2}\left(\frac{\alpha^{\prime}}{2}\right)+4 b \mu\left(u+u^{\prime}\right) \sin ^{2}\left(\frac{\alpha^{\prime}}{2}\right)+b^{2} \mu\left(4 u u^{\prime} \sin ^{2}\left(\frac{\alpha^{\prime}}{2}\right)+\left(u-u^{\prime}\right)^{2}\right)} d \mu \\
& +b^{2} \int_{0}^{1} \frac{\mu\left(u+u^{\prime}\right)\left(4 u u^{\prime} \sin ^{2}\left(\frac{\alpha^{\prime}}{2}\right)+\left(u-u^{\prime}\right)^{2}\right)}{4 \sin ^{2}\left(\frac{\alpha^{\prime}}{2}\right)+4 b \mu\left(u+u^{\prime}\right) \sin ^{2}\left(\frac{\alpha^{\prime}}{2}\right)+b^{2} \mu\left(4 u u^{\prime} \sin ^{2}\left(\frac{\alpha^{\prime}}{2}\right)+\left(u-u^{\prime}\right)^{2}\right)} d \mu .
\end{aligned}
$$

Therefore

$$
\left|\frac{1}{b} \log \left(\frac{A[a]}{A[0]}\right)-\left(u+u^{\prime}\right)\right| \leq C b \int_{0}^{1} \frac{\sin ^{2}\left(\frac{\alpha^{\prime}}{2}\right)+s^{\prime 2}}{\sin ^{2}\left(\frac{\alpha^{\prime}}{2}\right)+b^{2} \mu s^{\prime 2}} d \mu .
$$


Taking one derivative we have that

$$
\begin{aligned}
& \partial\left(\frac{1}{a^{2}} \log \left(\frac{A[a]}{A[0]}\right)-\left(u+u^{\prime}\right)\right)=\frac{1}{b} \frac{\partial A[a]}{A[a]}-\partial\left(u+u^{\prime}\right) \\
& =\frac{4 \partial\left(u+u^{\prime}\right) \sin ^{2}\left(\frac{\alpha^{\prime}}{2}\right)+b\left(4 \partial\left(u u^{\prime}\right) \sin ^{2}\left(\frac{\alpha^{\prime}}{2}\right)+2\left(u-u^{\prime}\right) \partial\left(u-u^{\prime}\right)\right)}{4 \sin ^{2}\left(\frac{\alpha^{\prime}}{2}\right)+4 b\left(u+u^{\prime}\right) \sin ^{2}\left(\frac{\alpha^{\prime}}{2}\right)+b^{2}\left(4 u u^{\prime} \sin ^{2}\left(\frac{\alpha^{\prime}}{2}\right)+\left(u-u^{\prime}\right)^{2}\right)}-\partial\left(u+u^{\prime}\right) \\
& =\frac{b\left(4 \partial\left(u u^{\prime}\right) \sin ^{2}\left(\frac{\alpha^{\prime}}{2}\right)+2\left(u-u^{\prime}\right) \partial\left(u-u^{\prime}\right)\right)-\partial\left(u+u^{\prime}\right)\left(4 b\left(u+u^{\prime}\right) \sin ^{2}\left(\frac{\alpha^{\prime}}{2}\right)+b^{2}\left(4 u u^{\prime} \sin ^{2}\left(\frac{\alpha^{\prime}}{2}\right)+\left(u-u^{\prime}\right)^{2}\right)\right)}{4 \sin ^{2}\left(\frac{\alpha^{\prime}}{2}\right)+4 b\left(u+u^{\prime}\right) \sin ^{2}\left(\frac{\alpha^{\prime}}{2}\right)+b^{2}\left(4 u u^{\prime} \sin ^{2}\left(\frac{\alpha^{\prime}}{2}\right)+\left(u-u^{\prime}\right)^{2}\right)}
\end{aligned}
$$

Therefore

$$
\left|\partial \frac{1}{b} \log \left(\frac{A[a]}{A[0]}\right)-\partial\left(u+u^{\prime}\right)\right| \leq C b \frac{\sin ^{2}\left(\frac{\alpha^{\prime}}{2}\right)+s^{\prime 2}}{\sin ^{2}\left(\frac{\alpha^{\prime}}{2}\right)+b^{2} s^{\prime 2}}
$$

Taking two derivatives we obtain

$$
\partial^{2}\left(\frac{1}{b} \log \left(\frac{A[a]}{A[0]}\right)-\left(u+u^{\prime}\right)\right)=\frac{1}{b} \frac{\partial^{2} A[a]}{A[a]}-\frac{1}{b} \frac{(\partial A[a])^{2}}{A[a]^{2}}-\partial^{2}\left(u+u^{\prime}\right)
$$

On one hand

$$
\begin{aligned}
& \frac{1}{b} \frac{\partial^{2} A[a]}{A[a]}-\partial^{2}\left(u+u^{\prime}\right) \\
& =b \frac{4 \partial^{2}\left(u u^{\prime}\right) \sin ^{2}\left(\frac{\alpha^{\prime}}{2}\right)+2\left(u-u^{\prime}\right) \partial^{2}\left(u-u^{\prime}\right)+2\left(\partial\left(u-u^{\prime}\right)\right)^{2}}{A[a]} \\
& +\frac{4 \partial^{2}\left(u+u^{\prime}\right) \sin ^{2}\left(\frac{\alpha^{\prime}}{2}\right)}{4 \sin ^{2}\left(\frac{\alpha^{\prime}}{2}\right)+4 b\left(u+u^{\prime}\right) \sin ^{2}\left(\frac{\alpha^{\prime}}{2}\right)+b^{2}\left(4 u u^{\prime} \sin ^{2}\left(\frac{\alpha^{\prime}}{2}\right)+\left(u-u^{\prime}\right)^{2}\right)}-\partial^{2}\left(u-u^{\prime}\right) \\
& =b \frac{4 \partial^{2}\left(u u^{\prime}\right) \sin ^{2}\left(\frac{\alpha^{\prime}}{2}\right)+2\left(u-u^{\prime}\right) \partial^{2}\left(u-u^{\prime}\right)+2\left(\partial\left(u-u^{\prime}\right)\right)^{2}}{A[a]} \\
& -b \frac{\left(4\left(u+u^{\prime}\right) \sin ^{2}\left(\frac{\alpha^{\prime}}{2}\right)+b\left(4 u u^{\prime} \sin ^{2}\left(\frac{\alpha^{\prime}}{2}\right)+\left(u-u^{\prime}\right)^{2}\right)\right) \partial^{2}\left(u+u^{\prime}\right)}{A[a]}
\end{aligned}
$$

And we have that

$$
\left|\frac{1}{b} \frac{\partial^{2} A[a]}{A[a]}-\partial^{2}\left(u+u^{\prime}\right)\right| \leq C b \frac{\sin ^{2}\left(\frac{\alpha^{\prime}}{2}\right)+s^{\prime 2}+\left|\sin \left(\frac{\alpha^{\prime}}{2}\right)\right|+\left|s^{\prime}\right|}{\sin ^{2}\left(\frac{\alpha^{\prime}}{2}\right)+b^{2} s^{\prime 2}}
$$

On the other hand

$$
\frac{(\partial A[a])^{2}}{A[a]^{2}}=b \frac{\left(4 \partial\left(u+u^{\prime}\right) \sin ^{2}\left(\frac{\alpha^{\prime}}{2}\right)+b\left(4 \partial\left(u u^{\prime}\right) \sin ^{2}\left(\frac{\alpha^{\prime}}{2}\right)+2\left(u-u^{\prime}\right) \partial\left(u-u^{\prime}\right)\right)\right)^{2}}{A[a]^{2}}
$$

and then

$$
\left|\frac{(\partial A[a])^{2}}{A[a]^{2}}\right| \leq C b \frac{\left(\sin ^{2}\left(\frac{\alpha^{\prime}}{2}\right)+b s^{\prime 2}\right)^{2}}{\left(\sin ^{2}\left(\frac{\alpha^{\prime}}{2}\right)+b^{2} s^{\prime 2}\right)^{2}}
$$


Taking three derivatives yields

$$
\partial^{3}\left(\frac{1}{a^{2}} \log \left(\frac{A[a]}{A[0]}\right)-\left(u+u^{\prime}\right)\right)=\frac{1}{b} \frac{\partial^{3} A[a]}{A[a]}-\partial^{3}\left(u+u^{\prime}\right)-\frac{3}{b} \frac{\partial^{2} A[a] \partial A[a]}{A[a]^{2}}+\frac{2}{b} \frac{(\partial A[a])^{3}}{A[a]^{3}} .
$$

The inequalities B.1 3 and B.1 4 yield

$$
\left|\frac{1}{b} \frac{\partial A[a] \partial^{2} A[a]}{A[a]^{2}}\right| \leq C b \frac{\left(\sin ^{2}\left(\frac{\alpha^{\prime}}{2}\right)+b s^{\prime 2}\right)\left(\sin ^{2}\left(\frac{\alpha^{\prime}}{2}\right)+b\left(\left|\sin \left(\frac{\alpha^{\prime}}{2}\right)+s^{\prime 2}+\right| s^{\prime} \mid\right)\right)}{\left(\sin ^{2}\left(\frac{\alpha^{\prime}}{2}\right)+b^{2} s^{\prime 2}\right)^{2}}
$$

and

$$
\left|\frac{1}{b} \frac{(\partial A[a])^{3}}{A[a]^{3}}\right| \leq C b^{2} \frac{\left(\sin ^{2}\left(\frac{\alpha^{\prime}}{2}\right)+b s^{\prime 3}\right)^{3}}{\left(\sin ^{2}\left(\frac{\alpha^{\prime}}{2}\right)+b^{2} s^{\prime 2}\right)^{3}}
$$

And B.14. 4 is then proven. To prove B.14.5 we notice that

$$
\begin{aligned}
& \frac{1}{b} \frac{\partial^{3} A[a]}{A[a]}-\partial^{3}\left(u+u^{\prime}\right)=\frac{4 \partial^{3}\left(u+u^{\prime}\right) \sin ^{2}\left(\frac{\alpha^{\prime}}{2}\right)}{A[a]}-\partial^{3}\left(u+u^{\prime}\right) \\
& +b \frac{4 \partial^{3}\left(u u^{\prime}\right) \sin ^{2}\left(\frac{\alpha^{\prime}}{2}\right)+2\left(u-u^{\prime}\right) \partial^{3}\left(u-u^{\prime}\right)}{A[a]} \\
& +b \frac{6 \partial\left(u-u^{\prime}\right) \partial^{2}\left(u-u^{\prime}\right)}{A[a]} .
\end{aligned}
$$

And we can compute

$$
\frac{4 \partial^{3}\left(u+u^{\prime}\right) \sin ^{2}\left(\frac{\alpha^{\prime}}{2}\right)}{A[a]}-\partial^{3}\left(u+u^{\prime}\right)=-b \frac{4\left(u+u^{\prime}\right) \sin ^{2}\left(\frac{\alpha^{\prime}}{2}\right)+b\left(4 u u^{\prime} \sin ^{2}\left(\frac{\alpha^{\prime}}{2}\right)+\left(u-u^{\prime}\right)^{2}\right)}{A[a]} \partial^{3}\left(u+u^{\prime}\right) .
$$

Thus

$$
\left|\frac{4 \partial^{3}\left(u+u^{\prime}\right) \sin ^{2}\left(\frac{\alpha^{\prime}}{2}\right)}{A[a]}-\partial^{3}\left(u+u^{\prime}\right)\right| \leq C b \frac{\sin ^{2}\left(\frac{\alpha^{\prime}}{2}\right)+b s^{\prime 2}}{\sin ^{2}\left(\frac{\alpha^{\prime}}{2}\right)+b^{2} s^{\prime 2}}\left|\partial^{3}\left(u+u^{\prime}\right)\right| .
$$

Therefore

$$
\begin{aligned}
& \left|\frac{1}{b} \frac{\partial^{3} A[a]}{A[a]}-\partial^{3}\left(u+u^{\prime}\right)\right| \leq C b \frac{\sin ^{2}\left(\frac{\alpha^{\prime}}{2}\right)+b s^{\prime 2}}{\sin ^{2}\left(\frac{\alpha^{\prime}}{2}\right)+b^{2} s^{\prime 2}}\left|\partial^{3}\left(u+u^{\prime}\right)\right| \\
& C b \frac{\sin ^{2}\left(\frac{\alpha^{\prime}}{2}\right)}{\sin ^{2}\left(\frac{\alpha^{\prime}}{2}\right)+b^{2} s^{\prime 2}}\left|\partial^{3}\left(u u^{\prime}\right)\right|+C b \frac{\left|\sin \left(\frac{\alpha^{\prime}}{2}\right)\right|+\left|s^{\prime}\right|}{\sin ^{2}\left(\frac{\alpha^{\prime}}{2}\right)+b^{2} s^{\prime 2}}\left|\partial^{3}\left(u-u^{\prime}\right)\right|+C b \frac{\left|\sin \left(\frac{\alpha^{\prime}}{2}\right)\right|+\left|s^{\prime}\right|}{\sin ^{2}\left(\frac{\alpha^{\prime}}{2}\right)+b^{2} s^{\prime 2}} .
\end{aligned}
$$

Lemma B.15 Let $g \in H^{4,3}$ with $\|g\|_{H^{4,3}}=1$ and $f(\alpha, s) \in L^{2}(\Omega)$ with $\operatorname{supp}(f) \subset \mathbb{T} \times[-1,1]$. Then the following estimates hold:

$$
\left\|\int_{-\infty}^{\infty} \int_{-\pi}^{\pi} \partial^{j}\left(\frac{1}{b} \log \left(\frac{A[a]}{A[0]}\right)-\left(u+u^{\prime}\right)\right)\left|\sin \left(\frac{\alpha^{\prime}}{2}\right)\right| f^{\prime} d \alpha^{\prime} d s^{\prime}\right\|_{L^{2}} \leq C b \log \left(\frac{1}{b}\right),
$$

for $j=0,1,2$. 


$$
\left\|\int_{-\infty}^{\infty} \int_{-\pi}^{\pi}\left(\partial^{3}\left(\frac{1}{b} \log \left(\frac{A[a]}{A[0]}\right)-\left(u+u^{\prime}\right)\right)-Z\left(s, s^{\prime}, \alpha, \alpha^{\prime}\right)\right)\left|\sin \left(\frac{\alpha^{\prime}}{2}\right)\right| f^{\prime} d \alpha^{\prime} d s^{\prime}\right\|_{L^{2}} \leq C b
$$

where

$$
Z\left(s, s^{\prime}, \alpha, \alpha^{\prime}\right)=\frac{1}{b} \frac{\partial^{3} A[a]}{A[a]}-\partial^{3}\left(u+u^{\prime}\right)
$$

Proof: The proof follows from Lemma B.14, Lemma B.2 and Minkowski's inequality.

Lemma B.16 Let $f(\alpha, s) \in L^{\infty}(\Omega)$ with $\operatorname{supp}(f) \subset \mathbb{T} \times[-1,1]$. Then the following estimates hold:

$$
\left\|\int_{-\infty}^{\infty} \int_{-\pi}^{\pi} Z\left(\alpha, \alpha^{\prime}, s, s^{\prime}\right)\left|\sin \left(\frac{\alpha^{\prime}}{2}\right)\right| f^{\prime} d \alpha^{\prime} d s^{\prime}\right\|_{L^{2}} \leq C b \log \left(\frac{1}{b}\right)
$$

where

$$
Z\left(\alpha, \alpha^{\prime}, s, s^{\prime}\right)=\frac{1}{b} \frac{\partial^{3} A[a]}{A[a]}-\partial^{3}\left(u+u^{\prime}\right)
$$

Proof: The proof follows from Lemma B.14, Lemma B.2 and Minkowski's inequality.

Lemma B.17 The following estimates hold:

1.

$$
\begin{aligned}
& \left|\partial_{a}\left(\frac{1}{a^{2}} \log \left(\frac{A[a]}{A[0]}\right)\right)\right| \\
& \leq C a \int_{0}^{1} \frac{\mu \sin ^{2}\left(\frac{\alpha^{\prime}}{2}\right)}{\sin ^{2}\left(\frac{\alpha^{\prime}}{2}\right)+(\sqrt{\mu} b)^{2} s^{\prime 2}} d \mu+C a^{3} \int_{0}^{1} \frac{\mu s^{\prime 2}}{\sin ^{2}\left(\frac{\alpha^{\prime}}{2}\right)+(\sqrt{\mu} b)^{2} s^{\prime 2}} d \mu \\
& +C a \frac{\sin ^{2}\left(\frac{\alpha^{\prime}}{2}\right)}{\sin ^{2}\left(\frac{\alpha^{\prime}}{2}\right)+b^{2} s^{\prime 2}}+C a^{3} \frac{s^{\prime 2}}{\sin ^{2}\left(\frac{\alpha^{\prime}}{2}\right)+b^{2} s^{\prime 2}} \\
& +C a \int_{0}^{1} \frac{\sin ^{2}\left(\frac{\alpha^{\prime}}{2}\right)+s^{\prime 2}}{\sin ^{2}\left(\frac{\alpha^{\prime}}{2}\right)+(\sqrt{\mu} b)^{2} s^{\prime 2}} d \mu \\
& +C a \frac{\sin ^{2}\left(\frac{\alpha^{\prime}}{2}\right)+s^{\prime 2}}{\sin ^{2}\left(\frac{\alpha^{\prime}}{2}\right)+b^{2} s^{\prime 2}}
\end{aligned}
$$

2.

$$
\left|\partial \partial_{a}\left(\frac{1}{a^{2}} \log \left(\frac{A[a]}{A[0]}\right)\right)\right| \leq C a \frac{\sin ^{2}\left(\frac{\alpha^{\prime}}{2}\right)+s^{\prime 2}}{\sin ^{2}\left(\frac{\alpha^{\prime}}{2}\right)+b^{2} s^{\prime 2}}+C a \frac{\left(\sin ^{2}\left(\frac{\alpha^{\prime}}{2}\right)+b s^{\prime 2}\right)^{2}}{\left(\sin ^{2}\left(\frac{\alpha^{\prime}}{2}\right)+b^{2} s^{\prime 2}\right)^{2}}
$$

3.

$$
\left|\partial^{2} \partial_{a}\left(\frac{1}{a^{2}} \log \left(\frac{A[a]}{A[0]}\right)\right)\right| \leq C a \frac{\left(\sin ^{2}\left(\frac{\alpha^{\prime}}{2}\right)+\left|\sin \left(\frac{\alpha^{\prime}}{2}\right)\right|+s^{\prime 2}+\left|s^{\prime}\right|\right)}{\sin ^{2}\left(\frac{\alpha^{\prime}}{2}\right)+b^{2} s^{\prime 2}}
$$




$$
\begin{aligned}
& +C a \frac{\left(\sin ^{2}\left(\frac{\alpha^{\prime}}{2}\right)+b s^{\prime 2}\right)^{2}}{\left(\sin ^{2}\left(\frac{\alpha^{\prime}}{2}\right)+b^{2} s^{\prime 2}\right)^{2}}+C a \frac{\left(\sin ^{2}\left(\frac{\alpha^{\prime}}{2}\right)+b s^{\prime 2}\right)\left(\sin ^{2}\left(\frac{\alpha^{\prime}}{2}\right)+b\left(\left|\sin \left(\frac{\alpha^{\prime}}{2}\right)\right|+s^{\prime 2}+\left|s^{\prime}\right|\right)\right)}{\left(\sin ^{2}\left(\frac{\alpha^{\prime}}{2}\right)+b^{2} s^{\prime 2}\right)^{2}} \\
& +C a^{3} \frac{\left(\sin ^{2}\left(\frac{\alpha^{\prime}}{2}\right)+b s^{\prime 2}\right)^{3}}{\left(\sin ^{2}\left(\frac{\alpha^{\prime}}{2}\right)+b^{2} s^{\prime 2}\right)^{3}} .
\end{aligned}
$$

4.

$$
\begin{aligned}
& \left|\partial^{3} \partial_{a}\left(\frac{1}{a^{2}} \log \left(\frac{A[a]}{A[0]}\right)\right)+\frac{2}{a^{2}} \frac{\partial^{3} A[a]}{A[a]}-\frac{1}{a^{2}} \frac{\partial^{3} \partial_{a} A[a]}{A[a]}+\frac{1}{a^{2}} \frac{\partial A[a] \partial^{3} A[a]}{A[a]^{2}}\right| \\
& \leq C a \frac{\left(\sin ^{2}\left(\frac{\alpha^{\prime}}{2}\right)+b s^{\prime 2}\right)\left(\sin ^{2}\left(\frac{\alpha^{\prime}}{2}\right)+b\left(\left|\sin \left(\frac{\alpha^{\prime}}{2}\right)\right|+s^{\prime 2}+\left|s^{\prime}\right|\right)\right)}{\left(\sin ^{2}\left(\frac{\alpha^{\prime}}{2}\right)+b^{2} s^{\prime 2}\right)^{2}} \\
& +C a^{3} \frac{\left(\sin ^{2}\left(\frac{\alpha^{\prime}}{2}\right)+b s^{\prime 2}\right)^{3}}{\left(\sin ^{2}\left(\frac{\alpha^{\prime}}{2}\right)+b^{2} s^{\prime 2}\right)^{3}}+C a \frac{\left(\sin ^{2}\left(\frac{\alpha^{\prime}}{2}\right)+b s^{\prime 2}\right)^{2}\left(\sin ^{2}\left(\frac{\alpha^{\prime}}{2}\right)+b\left(\left|\sin \left(\frac{\alpha^{\prime}}{2}\right)\right|+s^{\prime 2}+\left|s^{\prime}\right|\right)\right)}{\left(\sin ^{2}\left(\frac{\alpha^{\prime}}{2}\right)+b^{2} s^{\prime 2}\right)^{3}} \\
& +C a^{5} \frac{\left(\sin ^{2}\left(\frac{\alpha^{\prime}}{2}\right)+b s^{\prime 2}\right)^{4}}{\left(\sin ^{2}\left(\frac{\alpha^{\prime}}{2}\right)+b^{2} s^{\prime 2}\right)^{4}}
\end{aligned}
$$

5.

$$
\begin{aligned}
& \left|\frac{2}{a^{2}} \frac{\partial^{3} A[a]}{A[a]}-\frac{1}{a^{2}} \frac{\partial^{3} \partial_{a} A[a]}{A[a]}+\frac{1}{a^{2}} \frac{\partial A[a] \partial^{3} A[a]}{A[a]^{2}}\right| \leq C a \frac{\sin ^{2}\left(\frac{\alpha^{\prime}}{2}\right)}{\sin ^{2}\left(\frac{\alpha^{\prime}}{2}\right)+b^{2} s^{\prime 2}}\left|\partial^{3}\left(u u^{\prime}\right)\right| \\
& +C a \frac{\left|\sin \left(\frac{\alpha^{\prime}}{2}\right)\right|+\left|s^{\prime}\right|}{\sin ^{2}\left(\frac{\alpha^{\prime}}{2}\right)+b^{2} s^{\prime 2}}+C a \frac{\left|\sin \left(\frac{\alpha^{\prime}}{2}\right)\right|+\left|s^{\prime}\right|}{\sin ^{2}\left(\frac{\alpha^{\prime}}{2}\right)+b^{2} s^{\prime 2}}\left|\partial^{3}\left(u-u^{\prime}\right)\right| .
\end{aligned}
$$

Proof: First we notice that

$$
\partial_{a}\left(\frac{1}{a^{2}} \log \left(\frac{A[a]}{A[0]}\right)\right)=-\frac{2}{a^{3}} \log \left(\frac{A[a]}{A[0]}\right)+\frac{1}{a^{2}} \frac{\partial_{a} A[a]}{A[a]}
$$

We have that

$$
\begin{aligned}
& -\frac{2}{a^{3}} \log \left(\frac{A[a]}{A[0]}\right)=-\frac{2}{a^{3}} \int_{0}^{1} \frac{4 b\left(u+u^{\prime}\right) \sin ^{2}\left(\frac{\alpha^{\prime}}{2}\right)+b^{2}\left(4 u u^{\prime} \sin ^{2}\left(\frac{\alpha^{\prime}}{2}\right)+\left(u-u^{\prime}\right)^{2}\right)}{4 \sin \alpha^{\prime}+4 b \mu\left(u+u^{\prime}\right) \sin ^{2}\left(\frac{\alpha^{\prime}}{2}\right)+b^{2} \mu\left(4 u u^{\prime} \sin ^{2}\left(\frac{\alpha^{\prime}}{2}\right)+\left(u-u^{\prime}\right)^{2}\right)} d \mu \\
& =\int_{0}^{1} \frac{-8 \frac{1}{a}\left(u+u^{\prime}\right) \sin ^{2}\left(\frac{\alpha^{\prime}}{2}\right)-2 a\left(4 u u^{\prime} \sin ^{2}\left(\frac{\alpha^{\prime}}{2}\right)+\left(u-u^{\prime}\right)^{2}\right)}{4 \sin ^{2}\left(\frac{\alpha^{\prime}}{2}\right)+4 b \mu\left(u+u^{\prime}\right) \sin ^{2}\left(\frac{\alpha^{\prime}}{2}\right)+b^{2} \mu\left(4 u u^{\prime} \sin ^{2}\left(\frac{\alpha^{\prime}}{2}\right)+\left(u-u^{\prime}\right)^{2}\right)} d \mu .
\end{aligned}
$$

In addition

$$
\frac{1}{a^{2}} \frac{\partial_{a} A[a]}{A[a]}=\frac{8 \frac{1}{a}\left(u+u^{\prime}\right) \sin ^{2}\left(\frac{\alpha^{\prime}}{2}\right)+4 a\left(4 u u^{\prime} \sin ^{2}\left(\frac{\alpha^{\prime}}{2}\right)+\left(u-u^{\prime}\right)^{2}\right)}{A[a]}
$$


Thus

$$
\begin{aligned}
& -\frac{2}{a^{3}} \log \left(\frac{A[a]}{A[0]}\right)+\frac{1}{a^{2}} \frac{\partial_{a} A[a]}{A[a]} \\
& =\frac{8}{a} \sin ^{2}\left(\frac{\alpha^{\prime}}{2}\right) \int_{0}^{1}\left(\frac{1}{4 \sin ^{2}\left(\frac{\alpha^{\prime}}{2}\right)+4 b \mu\left(u+u^{\prime}\right) \sin ^{2}\left(\frac{\alpha^{\prime}}{2}\right)+b^{2} \mu\left(4 u u^{\prime} \sin ^{2}\left(\frac{\alpha^{\prime}}{2}\right)+\left(u-u^{\prime}\right)^{2}\right)}-\frac{1}{A[a]}\right) d \mu \\
& +\int_{0}^{1} \frac{-2 a\left(4 u u^{\prime} \sin ^{2}\left(\frac{\alpha^{\prime}}{2}\right)+\left(u-u^{\prime}\right)^{2}\right)}{4 \sin ^{2}\left(\frac{\alpha^{\prime}}{2}\right)+4 b \mu\left(u+u^{\prime}\right) \sin ^{2}\left(\frac{\alpha^{\prime}}{2}\right)+b^{2} \mu\left(4 u u^{\prime} \sin ^{2}\left(\frac{\alpha^{\prime}}{2}\right)+\left(u-u^{\prime}\right)^{2}\right)} d \mu \\
& +\frac{4 a\left(4 u u^{\prime} \sin ^{2}\left(\frac{\alpha^{\prime}}{2}\right)+\left(u-u^{\prime}\right)^{2}\right)}{A[a]}
\end{aligned}
$$

We will split the first term of the right hand side of the previous expression in the following way

$$
\begin{aligned}
& \frac{8}{a} \sin ^{2}\left(\frac{\alpha^{\prime}}{2}\right) \int_{0}^{1}\left(\frac{1}{4 \sin ^{2}\left(\frac{\alpha^{\prime}}{2}\right)+4 b \mu\left(u+u^{\prime}\right) \sin ^{2}\left(\frac{\alpha^{\prime}}{2}\right)+b^{2} \mu\left(4 u u^{\prime} \sin ^{2}\left(\frac{\alpha^{\prime}}{2}\right)+\left(u-u^{\prime}\right)^{2}\right)}-\frac{1}{A[a]}\right) d \mu \\
& =\frac{8}{a} \sin ^{2}\left(\frac{\alpha^{\prime}}{2}\right) \int_{0}^{1}\left(\frac{1}{4 \sin ^{2}\left(\frac{\alpha^{\prime}}{2}\right)+4 b \mu\left(u+u^{\prime}\right) \sin ^{2}\left(\frac{\alpha^{\prime}}{2}\right)+b^{2} \mu\left(4 u u^{\prime} \sin ^{2}\left(\frac{\alpha^{\prime}}{2}\right)+\left(u-u^{\prime}\right)^{2}\right)}-\frac{1}{4 \sin ^{2}\left(\frac{\alpha^{\prime}}{2}\right)}\right) d \mu \\
& +\frac{8}{a} \sin ^{2}\left(\frac{\alpha^{\prime}}{2}\right)\left(\frac{1}{4 \sin ^{2}\left(\frac{\alpha^{\prime}}{2}\right)}-\frac{1}{A[a]}\right) .
\end{aligned}
$$

And we can estimate

$$
\begin{aligned}
& \left|\frac{8}{a} \sin ^{2}\left(\frac{\alpha^{\prime}}{2}\right) \int_{0}^{1}\left(\frac{1}{4 \sin ^{2}\left(\frac{\alpha^{\prime}}{2}\right)+4 b \mu\left(u+u^{\prime}\right) \sin ^{2}\left(\frac{\alpha^{\prime}}{2}\right)+b^{2} \mu\left(4 u u^{\prime} \sin ^{2}\left(\frac{\alpha^{\prime}}{2}\right)+\left(u-u^{\prime}\right)^{2}\right)}-\frac{1}{4 \sin ^{2}\left(\frac{\alpha^{\prime}}{2}\right)}\right) d \mu\right| \\
& \leq 2\left|\int_{0}^{1} \frac{4 a \mu\left(u+u^{\prime}\right) \sin ^{2}\left(\frac{\alpha^{\prime}}{2}\right)+a^{3} \mu\left(4 u u^{\prime} \sin ^{2}\left(\frac{\alpha^{\prime}}{2}\right)+\left(u-u^{\prime}\right)^{2}\right)}{4 \sin ^{2}\left(\frac{\alpha^{\prime}}{2}\right)+4 b \mu\left(u+u^{\prime}\right) \sin ^{2}\left(\frac{\alpha^{\prime}}{2}\right)+b^{2} \mu\left(4 u u^{\prime} \sin ^{2}\left(\frac{\alpha^{\prime}}{2}\right)+\left(u-u^{\prime}\right)^{2}\right)} d \mu\right| \\
& \leq C a \int_{0}^{1} \frac{\mu \sin ^{2}\left(\frac{\alpha^{\prime}}{2}\right)}{\sin ^{2}\left(\frac{\alpha^{\prime}}{2}\right)+(\sqrt{\mu} b)^{2} s^{\prime 2}} d \mu+C a^{3} \int_{0}^{1} \frac{\mu\left(\sin ^{2}\left(\frac{\alpha^{\prime}}{2}\right)+s^{\prime 2}\right)}{\sin ^{2}\left(\frac{\alpha^{\prime}}{2}\right)+(\sqrt{\mu} b)^{2} s^{\prime 2}} d \mu \\
& \leq C a \int_{0}^{1} \frac{\mu \sin ^{2}\left(\frac{\alpha^{\prime}}{2}\right)}{\sin ^{2}\left(\frac{\alpha^{\prime}}{2}\right)+(\sqrt{\mu} b)^{2} s^{\prime 2}} d \mu+C a^{3} \int_{0}^{1} \frac{\mu s^{\prime 2}}{\sin ^{2}\left(\frac{\alpha^{\prime}}{2}\right)+(\sqrt{\mu} b)^{2} s^{\prime 2}} d \mu,
\end{aligned}
$$

where we have used (B.5). In a similar way

$$
\left|\frac{8}{a} \sin ^{2}\left(\frac{\alpha^{\prime}}{2}\right)\left(\frac{1}{4 \sin ^{2}\left(\frac{\alpha^{\prime}}{2}\right)}-\frac{1}{A[a]}\right)\right| \leq C a \frac{\sin ^{2}\left(\frac{\alpha^{\prime}}{2}\right)}{\sin ^{2}\left(\frac{\alpha^{\prime}}{2}\right)+b^{2} s^{\prime 2}}+C a^{3} \frac{s^{\prime 2}}{\sin ^{2}\left(\frac{\alpha^{\prime}}{2}\right)+b^{2} s^{\prime 2}} .
$$

In addition we have that

$$
\begin{aligned}
& \left|\int_{0}^{1} \frac{-2 a\left(4 u u^{\prime} \sin ^{2}\left(\frac{\alpha^{\prime}}{2}\right)+\left(u-u^{\prime}\right)^{2}\right)}{4 \sin ^{2}\left(\frac{\alpha^{\prime}}{2}\right)+4 b \mu\left(u+u^{\prime}\right) \sin ^{2}\left(\frac{\alpha^{\prime}}{2}\right)+b^{2} \mu\left(4 u u^{\prime} \sin ^{2}\left(\frac{\alpha^{\prime}}{2}\right)+\left(u-u^{\prime}\right)^{2}\right)} d \mu\right| \\
& \leq C a \int_{0}^{1} \frac{\sin ^{2}\left(\frac{\alpha^{\prime}}{2}\right)+s^{\prime 2}}{\sin ^{2}\left(\frac{\alpha^{\prime}}{2}\right)+(\sqrt{\mu} b)^{2} s^{\prime 2}} d \mu
\end{aligned}
$$




$$
\left|\frac{4 a\left(4 u u^{\prime} \sin ^{2}\left(\frac{\alpha^{\prime}}{2}\right)+\left(u-u^{\prime}\right)^{2}\right)}{A[a]}\right| \leq C a \frac{\sin ^{2}\left(\frac{\alpha^{\prime}}{2}\right)+s^{\prime 2}}{\sin ^{2}\left(\frac{\alpha^{\prime}}{2}\right)+b^{2} s^{\prime 2}},
$$

and we have already proven the first inequality of the lemma.

Taking one derivative we have that

$$
\partial \partial_{a}\left(\frac{1}{a^{2}} \log \left(\frac{A[a]}{A[0]}\right)\right)=-\frac{2}{a^{3}} \frac{\partial A[a]}{A[a]}+\frac{1}{a^{2}} \frac{\partial \partial_{a} A[a]}{A[a]}-\frac{1}{a^{2}} \frac{\partial_{a} A[a] \partial A[a]}{A[a]^{2}} .
$$

On one hand, we can compute that

$$
-\frac{2}{a^{3}} \frac{\partial A[a]}{A[a]}+\frac{1}{a^{2}} \frac{\partial \partial_{a} A[a]}{A[a]}=2 a \frac{4 \partial\left(u u^{\prime}\right) \sin ^{2}\left(\frac{\alpha^{\prime}}{2}\right)+2\left(u-u^{\prime}\right) \partial\left(u-u^{\prime}\right)}{A[a]}
$$

and then

$$
\left|-\frac{2}{a^{3}} \frac{\partial A[a]}{A[a]}+\frac{1}{a^{2}} \frac{\partial \partial_{a} A[a]}{A[a]}\right| \leq C a \frac{\sin ^{2}\left(\frac{\alpha^{\prime}}{2}\right)+s^{\prime 2}}{\sin ^{2}\left(\frac{\alpha^{\prime}}{2}\right)+b^{2} s^{\prime 2}} .
$$

On the other hand

$$
\left|\frac{1}{a^{2}} \frac{\partial_{a} A[a] \partial A[a]}{A[a]^{2}}\right| \leq C a \frac{\left(\sin ^{2}\left(\frac{\alpha^{\prime}}{2}\right)+b s^{\prime 2}\right)^{2}}{\left(\sin ^{2}\left(\frac{\alpha^{\prime}}{2}\right)+b^{2} s^{\prime 2}\right)^{2}}
$$

Therefore

$$
\left|\partial \partial_{a}\left(\frac{1}{a^{2}} \log \left(\frac{A[a]}{A[0]}\right)\right)\right| \leq C a \frac{\sin ^{2}\left(\frac{\alpha^{\prime}}{2}\right)+s^{\prime 2}}{\sin ^{2}\left(\frac{\alpha^{\prime}}{2}\right)+b^{2} s^{\prime 2}}+C a \frac{\left(\sin ^{2}\left(\frac{\alpha^{\prime}}{2}\right)+b s^{\prime 2}\right)^{2}}{\left(\sin ^{2}\left(\frac{\alpha^{\prime}}{2}\right)+b^{2} s^{\prime 2}\right)^{2}}
$$

Taking two derivatives we have that

$$
\begin{aligned}
& \partial^{2} \partial_{a}\left(\frac{1}{a^{2}} \log \left(\frac{A[a]}{A[0]}\right)\right)=-\frac{2}{a^{3}} \frac{\partial^{2} A[a]}{A[a]}+\frac{2}{a^{3}} \frac{\partial A[a]^{2}}{A[a]^{2}}+\frac{1}{a^{2}} \frac{\partial^{2} \partial_{a} A[a]}{A[a]}-\frac{2}{a^{2}} \frac{\partial \partial_{a} A[a] \partial A[a]}{A[a]^{2}} \\
& -\frac{1}{a^{2}} \frac{\partial_{a} A[a] \partial^{2} A[a]}{A[a]^{2}}+\frac{2}{a^{2}} \frac{\partial_{a} A[a] \partial A[a]^{2}}{A[a]^{3}} .
\end{aligned}
$$

And we can estimate

$$
\begin{aligned}
& \left|-\frac{2}{a^{3}} \frac{\partial^{2} A[a]}{A[a]}+\frac{1}{a^{2}} \frac{\partial^{2} \partial_{a} A[a]}{A[a]}\right| \leq C a \frac{\left(\sin ^{2}\left(\frac{\alpha^{\prime}}{2}\right)+\left|\sin \left(\frac{\alpha^{\prime}}{2}\right)\right|+s^{\prime 2}+\left|s^{\prime}\right|\right)}{\sin ^{2}\left(\frac{\alpha^{\prime}}{2}\right)+b^{2} s^{\prime 2}} \\
& \left|\frac{2}{a^{3}} \frac{\partial A[a]^{2}}{A[a]^{2}}\right| \leq C a \frac{\left(\sin ^{2}\left(\frac{\alpha^{\prime}}{2}\right)+b s^{\prime 2}\right)^{2}}{\left(\sin ^{2}\left(\frac{\alpha^{\prime}}{2}\right)+b^{2} s^{\prime 2}\right)^{2}} \\
& \left|\frac{2}{a^{2}} \frac{\partial \partial_{a} A[a] \partial A[a]}{A[a]^{2}}\right| \leq C a \frac{\left(\sin ^{2}\left(\frac{\alpha^{\prime}}{2}\right)+b s^{\prime 2}\right)^{2}}{\left(\sin ^{2}\left(\frac{\alpha^{\prime}}{2}\right)+b^{2} s^{\prime 2}\right)^{2}} \\
& \left|\frac{1}{a^{2}} \frac{\partial_{a} A[a] \partial^{2} A[a]}{A[a]^{2}}\right| \leq C a \frac{\left(\sin ^{2}\left(\frac{\alpha^{\prime}}{2}\right)+b s^{\prime 2}\right)\left(\sin ^{2}\left(\frac{\alpha^{\prime}}{2}\right)+b\left(\left|\sin \left(\frac{\alpha^{\prime}}{2}\right)\right|+s^{\prime 2}+\left|s^{\prime}\right|\right)\right)}{\left(\sin ^{2}\left(\frac{\alpha^{\prime}}{2}\right)+b^{2} s^{\prime 2}\right)^{2}}
\end{aligned}
$$




$$
\left|\frac{2}{a^{2}} \frac{\partial_{a} A[a] \partial A[a]^{2}}{A[a]^{3}}\right| \leq C a^{3} \frac{\left(\sin ^{2}\left(\frac{\alpha^{\prime}}{2}\right)+b s^{\prime 2}\right)^{3}}{\left(\sin ^{2}\left(\frac{\alpha^{\prime}}{2}\right)+b^{2} s^{\prime 2}\right)^{3}}
$$

This proves the third inequality.

Taking three derivatives yields

$$
\begin{aligned}
& \partial^{3} \partial_{a}\left(\frac{1}{a^{2}} \log \left(\frac{A[a]}{A[0]}\right)\right)=-\frac{2}{a^{3}} \frac{\partial^{3} A[a]}{A[a]}+\frac{6}{a^{3}} \frac{\partial^{2} A[a] \partial A[a]}{A[a]^{2}}-\frac{4}{a^{3}} \frac{(\partial A[a])^{3}}{A[a]^{3}}+\frac{1}{a^{2}} \frac{\partial^{3} \partial_{a} A[a]}{A[a]} \\
& -\frac{3}{a^{2}} \frac{\partial^{2} \partial_{a} A[a] \partial A[a]}{A[a]^{2}}-\frac{3}{a^{2}} \frac{\partial \partial_{a} A[a] \partial^{2} A[a]}{A[a]^{2}}+\frac{6}{a^{2}} \frac{\partial \partial_{a} A[a](\partial A[a])^{2}}{A[a]^{3}}-\frac{1}{a^{2}} \frac{\partial_{a} A[a] \partial^{3} A[a]}{A[a]^{2}} \\
& +\frac{6}{a^{2}} \frac{\partial A[a] \partial^{2} A[a] \partial A[a]}{A[a]^{3}}-\frac{6}{a^{2}} \frac{\partial_{a} A[a](\partial A[a])^{3}}{A[a]^{4}} .
\end{aligned}
$$

And we can estimate

$$
\begin{aligned}
& \left|\frac{6}{a^{3}} \frac{\partial^{2} A[a] \partial A[a]}{A[a]^{2}}\right| \leq C a \frac{\left(\sin ^{2}\left(\frac{\alpha^{\prime}}{2}\right)+b s^{\prime 2}\right)\left(\sin ^{2}\left(\frac{\alpha^{\prime}}{2}\right)+b\left(\left|\sin \left(\frac{\alpha^{\prime}}{2}\right)\right|+s^{\prime 2}+\left|s^{\prime}\right|\right)\right)}{\left(\sin ^{2}\left(\frac{\alpha^{\prime}}{2}\right)+b^{2} s^{\prime 2}\right)^{2}} \\
& \left|\frac{4}{a^{3}} \frac{(\partial A[a])^{3}}{A[a]^{3}}\right| \leq C a^{3} \frac{\left(\sin ^{2}\left(\frac{\alpha^{\prime}}{2}\right)+b s^{\prime 2}\right)^{3}}{\left(\sin ^{2}\left(\frac{\alpha^{\prime}}{2}\right)+b^{2} s^{\prime 2}\right)^{3}} \\
& \left|\frac{3}{a^{2}} \frac{\partial^{2} \partial_{a} A[a] \partial A[a]}{A[a]^{2}}\right| \leq C a \frac{\left(\sin ^{2}\left(\frac{\alpha^{\prime}}{2}\right)+b s^{\prime 2}\right)\left(\sin ^{2}\left(\frac{\alpha^{\prime}}{2}\right)+b\left(\left|\sin \left(\frac{\alpha^{\prime}}{2}\right)\right|+s^{\prime 2}+\left|s^{\prime}\right|\right)\right)}{\left(\sin ^{2}\left(\frac{\alpha^{\prime}}{2}\right)+b^{2} s^{\prime 2}\right)^{2}} \\
& \left|\frac{3}{a^{2}} \frac{\partial \partial_{a} A[a] \partial^{2} A[a]}{A[a]^{2}}\right| \leq C a \frac{\left(\sin ^{2}\left(\frac{\alpha^{\prime}}{2}\right)+b s^{\prime 2}\right)\left(\sin ^{2}\left(\frac{\alpha^{\prime}}{2}\right)+b\left(\left|\sin \left(\frac{\alpha^{\prime}}{2}\right)\right|+s^{\prime 2}+\left|s^{\prime}\right|\right)\right)}{\left(\sin ^{2}\left(\frac{\alpha^{\prime}}{2}\right)+b^{2} s^{\prime 2}\right)^{2}} \\
& \left|\frac{6}{a^{2}} \frac{\partial \partial_{a} A[a](\partial A[a])^{2}}{A[a]^{3}}\right| \leq C a^{3} \frac{\left(\sin ^{2}\left(\frac{\alpha^{\prime}}{2}\right)+b s^{\prime 2}\right)^{3}}{\left(\sin ^{2}\left(\frac{\alpha^{\prime}}{2}\right)+b^{2} s^{\prime 2}\right)^{3}} \\
& \left|\frac{6}{a^{2}} \frac{\partial_{a} A[a] \partial^{2} A[a] \partial A[a]}{A[a]^{3}}\right| \leq C a \frac{\left(\sin ^{2}\left(\frac{\alpha^{\prime}}{2}\right)+b s^{\prime 2}\right)^{2}\left(\sin ^{2}\left(\frac{\alpha^{\prime}}{2}\right)+b\left(\left|\sin \left(\frac{\alpha^{\prime}}{2}\right)\right|+s^{\prime 2}+\left|s^{\prime}\right|\right)\right)}{\left(\sin ^{2}\left(\frac{\alpha^{\prime}}{2}\right)+b^{2} s^{\prime 2}\right)^{3}} \\
& \left|\frac{6}{a^{2}} \frac{\partial_{a} A[a](\partial A[a])^{3}}{A[a]^{4}}\right| \leq C a^{5} \frac{\left(\sin ^{2}\left(\frac{\alpha^{\prime}}{2}\right)+b s^{\prime 2}\right)^{4}}{\left(\sin ^{2}\left(\frac{\alpha^{\prime}}{2}\right)+b^{2} s^{\prime 2}\right)^{4}} \cdot
\end{aligned}
$$

Then we have proven the fourth inequality.

Finally we notice that

$$
\begin{aligned}
& \left|-\frac{2}{a^{3}} \frac{\partial^{3} A[a]}{A[a]}+\frac{1}{a^{2}} \frac{\partial^{3} \partial_{a} A[a]}{A[a]}\right| \leq C a \frac{\sin ^{2}\left(\frac{\alpha^{\prime}}{2}\right)}{\sin ^{2}\left(\frac{\alpha^{\prime}}{2}\right)+b^{2} s^{\prime 2}}\left|\partial^{3}\left(u u^{\prime}\right)\right| \\
& +C a \frac{\left|\sin \left(\frac{\alpha^{\prime}}{2}\right)\right|+\left|s^{\prime}\right|}{\sin ^{2}\left(\frac{\alpha^{\prime}}{2}\right)+b^{2} s^{\prime 2}}+C a \frac{\left|\sin \left(\frac{\alpha^{\prime}}{2}\right)\right|+\left|s^{\prime}\right|}{\sin ^{2}\left(\frac{\alpha^{\prime}}{2}\right)+b^{2} s^{\prime 2}}\left|\partial^{3}\left(u-u^{\prime}\right)\right| \\
& \left|\frac{1}{a^{2}} \frac{\partial_{a} A[a] \partial^{3} A[a]}{A[a]^{2}}\right|
\end{aligned}
$$




$$
\begin{aligned}
& \left|\frac{1}{a^{2}} \frac{\partial_{a} A[a] \partial^{3} A[a]}{A[a]}\right| \leq C a \frac{\sin ^{2}\left(\frac{\alpha^{\prime}}{2}\right)+b s^{\prime 2}}{\sin ^{2}\left(\frac{\alpha^{\prime}}{2}\right)+b^{2} s^{\prime 2}} \sin ^{2}\left(\frac{\alpha^{\prime}}{2}\right)\left|\partial^{3}\left(u+u^{\prime}\right)\right| \\
& +C a^{3} \frac{\sin ^{2}\left(\frac{\alpha^{\prime}}{2}\right)+b s^{\prime 2}}{\sin ^{2}\left(\frac{\alpha^{\prime}}{2}\right)+b^{2} s^{\prime 2}} \sin ^{2}\left(\frac{\alpha^{\prime}}{2}\right)\left|\partial^{3}\left(u u^{\prime}\right)\right|+C a^{3} \frac{\sin ^{2}\left(\frac{\alpha^{\prime}}{2}\right)+b s^{\prime 2}}{\sin ^{2}\left(\frac{\alpha^{\prime}}{2}\right)+b^{2} s^{\prime 2}}\left(\left|\sin \left(\frac{\alpha^{\prime}}{2}\right)\right|+\left|s^{\prime}\right|\right) \\
& +C a^{3} \frac{\sin ^{2}\left(\frac{\alpha^{\prime}}{2}\right)+b s^{\prime 2}}{\sin ^{2}\left(\frac{\alpha^{\prime}}{2}\right)+b^{2} s^{\prime 2}}\left(\left|\sin \left(\frac{\alpha^{\prime}}{2}\right)\right|+\left|s^{\prime}\right|\right)\left|\partial^{3}\left(u-u^{\prime}\right)\right| .
\end{aligned}
$$

\section{Lemma B.18}

$$
\left\|\int_{-\infty}^{\infty} \partial^{j}\left(\partial_{a} \frac{1}{a^{2}} \log \left(\frac{A[a]}{A[0]}\right)\right)\left|\sin \left(\frac{\alpha^{\prime}}{2}\right)\right| f^{\prime} d \alpha^{\prime} d s^{\prime}\right\|_{L^{2}} \leq C a \log \left(\frac{1}{b}\right)
$$

for $j=0,1,2$.

Proof: The proof follows from Lemma B.17 Lemma B.2 and Minkowski's inequality.

\section{Lemma B.19}

$$
\left\|\int_{-\infty}^{\infty} \int_{-\pi}^{\pi} \partial^{3}\left(\partial_{a} \frac{1}{a^{2}} \log \left(\frac{A[a]}{A[0]}\right)\right)\left|\sin \left(\frac{\alpha^{\prime}}{2}\right)\right| f^{\prime} d \alpha^{\prime} d s^{\prime}\right\|_{L^{2}} \leq C a \log \left(\frac{1}{b}\right)
$$

Proof: The proof follows from Lemma B.17, Lemma B.2 and Minkowski's inequality.

Lemma B.20 Let $g \in H^{4,3}$ with $\|g\|_{H^{4,3}}=1$ then the following estimates hold

2.

$$
\left|A^{\prime}[u+t g]\right| \geq c\left(\sin ^{2}\left(\frac{\alpha^{\prime}}{2}\right)+b^{2} s^{\prime 2}\right)
$$

$$
\left|\partial_{u} A^{\prime}[g]\right| \leq C b\left(\sin ^{2}\left(\frac{\alpha^{\prime}}{2}\right)+b s^{\prime 2}\right)
$$

3.

$$
\left|A^{\prime}[u+t g]-A^{\prime}[u]\right| \leq C b t\left(\sin ^{2}\left(\frac{\alpha^{\prime}}{2}\right)+b s^{\prime 2}\right) .
$$

4 .

$$
\left|\partial A^{\prime}[u+t g]-\partial A^{\prime}[u]\right| \leq C b t\left(\sin ^{2}\left(\frac{\alpha^{\prime}}{2}\right)+b s^{\prime 2}\right) .
$$

5.

$$
\left|\partial^{2} A^{\prime}[u+t g]-\partial^{2} A^{\prime}[u]\right| \leq C b t\left(\sin ^{2}\left(\frac{\alpha^{\prime}}{2}\right)+b\left(\left|\sin \left(\frac{\alpha^{\prime}}{2}\right)\right|+\left|s^{\prime}\right|+s^{\prime 2}\right)\right) .
$$


6 .

$$
\left|A^{\prime}[u+t g]-A^{\prime}[u]-t \partial_{u} A[g]\right| \leq C t^{2} b^{2}\left(\sin ^{2}\left(\frac{\alpha^{\prime}}{2}\right)+s^{\prime 2}\right) .
$$

7.

$$
\left|\partial A^{\prime}[u+t g]-\partial A^{\prime}[u]-t \partial \partial_{u} A[g]\right| \leq C t^{2} b^{2}\left(\sin ^{2}\left(\frac{\alpha^{\prime}}{2}\right)+s^{\prime 2}\right)
$$

8.

$$
\left|\partial^{2}\left(A^{\prime}[u+t g]-A^{\prime}[u]-t \partial_{u} A^{\prime}[u]\right)\right| \leq C t^{2} b^{2}\left(\sin ^{2}\left(\frac{\alpha^{\prime}}{2}\right)+s^{\prime 2}+\left|\sin \left(\frac{\alpha^{\prime}}{2}\right)\right|+\left|s^{\prime}\right|\right)
$$

Proof: We recall that

$$
\begin{aligned}
A^{\prime}[u+t g] & =4 \sin ^{2}\left(\frac{\alpha^{\prime}}{2}\right)+4 b\left(u+u^{\prime}+t\left(g+g^{\prime}\right)\right) \sin ^{2}\left(\frac{\alpha^{\prime}}{2}\right) \\
& +b^{2}\left(4(u+t g)\left(u^{\prime}+t g^{\prime}\right) \sin ^{2}\left(\frac{\alpha^{\prime}}{2}\right)+\left(u-u^{\prime}+t\left(g-g^{\prime}\right)\right)^{2}\right) \\
& =A^{\prime}[u]+t\left(b\left(g+g^{\prime}\right) \sin ^{2}\left(\frac{\alpha^{\prime}}{2}\right)+4\left(\left(u g^{\prime}+u^{\prime} g\right) \sin ^{2}\left(\frac{\alpha^{\prime}}{2}\right)+2\left(u-u^{\prime}\right)\left(g-g^{\prime}\right)\right) b^{2}\right) \\
& +t^{2} b^{2}\left(4 g g^{\prime} \sin ^{2}\left(\frac{\alpha^{\prime}}{2}\right)+\left(g-g^{\prime}\right)^{2}\right) .
\end{aligned}
$$

Therefore

$$
A^{\prime}[u+t g] \geq A^{\prime}[u]-t c\left(b+b^{2}\right) \sin ^{2}\left(\frac{\alpha^{\prime}}{2}\right)-c t^{2} b^{2}\left(\sin ^{2}\left(\frac{\alpha^{\prime}}{2}\right)+s^{\prime 2}\right) .
$$

And for $t$ small enough $(b \in[0,1])$

$$
A^{\prime}[u+t g] \geq c\left(\sin ^{2}\left(\frac{\alpha^{\prime}}{2}\right)+b^{2} s^{\prime 2}\right) .
$$

To prove the second inequality in the statement of the lemma we recall that

$$
\partial_{u} A^{\prime}[g]=b\left(g+g^{\prime}\right) \sin ^{2}\left(\frac{\alpha^{\prime}}{2}\right)+4 b^{2}\left(\left(u g^{\prime}+u^{\prime} g\right) \sin ^{2}\left(\frac{\alpha^{\prime}}{2}\right)+2\left(u-u^{\prime}\right)\left(g-g^{\prime}\right)\right)
$$

and then

$$
\left|\partial_{u} A^{\prime}\right| \leq C\left(b \sin ^{2}\left(\frac{\alpha^{\prime}}{2}\right)+b^{2} s^{\prime 2}\right) .
$$

The third, fourth and fifth inequalities are straightforward. Taking two derivatives we have that

$$
\begin{aligned}
& \partial^{2} A^{\prime}[u+t g]-\partial^{2} A^{\prime}[u] \\
& =t b \partial^{2}\left(g+g^{\prime}\right) \sin ^{2}\left(\frac{\alpha^{\prime}}{2}\right) \\
& +t 4 b^{2}\left(\partial^{2}\left(u g^{\prime}+u^{\prime} g\right) \sin ^{2}\left(\frac{\alpha^{\prime}}{2}\right)+2 \partial^{2}\left(u-u^{\prime}\right)\left(g-g^{\prime}\right)+4 \partial\left(u-u^{\prime}\right) \partial\left(g-g^{\prime}\right)+2\left(u-u^{\prime}\right) \partial^{2}\left(g-g^{\prime}\right)\right)
\end{aligned}
$$




$$
+t^{2} b^{2}\left(4 \partial^{2}\left(g g^{\prime}\right) \sin ^{2}\left(\frac{\alpha^{\prime}}{2}\right)+2\left(\partial g-\partial g^{\prime}\right)^{2}+2\left(g-g^{\prime}\right) \partial^{2}\left(g-g^{\prime}\right)\right)
$$

and we can bound

$$
\begin{aligned}
& \left|\partial^{2} A^{\prime}[u+t g]-\partial^{2} A^{\prime}[u]\right| \leq t\left(b \sin ^{2}\left(\frac{\alpha^{\prime}}{2}\right)+b^{2}\left(\sin ^{2}\left(\frac{\alpha^{\prime}}{2}\right)+\left|\sin \frac{\alpha^{\prime}}{2}\right|+s^{\prime 2}+\left|s^{\prime}\right|\right)\right) \\
& +t^{2} b^{2}\left(\sin ^{2}\left(\frac{\alpha^{\prime}}{2}\right)+\left|\sin \frac{\alpha^{\prime}}{2}\right|+s^{\prime 2}+\left|s^{\prime}\right|\right) .
\end{aligned}
$$

To prove 6,7 and 8 we just notice that

$$
A^{\prime}[u+t g]-A^{\prime}[u]-t \partial_{u} A^{\prime}[g]=t^{2} b^{2}\left(4 g g^{\prime} \sin ^{2}\left(\frac{\alpha^{\prime}}{2}\right)+\left(g-g^{\prime}\right)^{2}\right) .
$$

Lemma B.21 The following estimates hold:

1.

$$
\begin{aligned}
& \left|\frac{1}{t} \log \left(\frac{A^{\prime}[u+t g]}{A^{\prime}[u]}\right)-\frac{\partial_{u} A^{\prime}[g]}{A^{\prime}[u]}\right| \\
& \leq C \frac{b \sin ^{2}\left(\frac{\alpha^{\prime}}{2}\right)+b^{2} s^{\prime 2}}{\sin ^{2}\left(\frac{\alpha^{\prime}}{2}\right)+b^{2} s^{\prime 2}}\left(t \frac{b \sin ^{2}\left(\frac{\alpha^{\prime}}{2}\right)+b^{2} s^{\prime 2}}{\sin ^{2}\left(\frac{\alpha^{\prime}}{2}\right)+b^{2} s^{\prime 2}}+t^{2} b^{2} \frac{\sin ^{2}\left(\frac{\alpha^{\prime}}{2}\right)+s^{\prime 2}}{\sin ^{2}\left(\frac{\alpha^{\prime}}{2}\right)+b^{2} s^{\prime 2}}\right)+C b^{2} t \frac{\sin ^{2}\left(\frac{\alpha^{\prime}}{2}\right)+s^{\prime 2}}{\sin ^{2}\left(\frac{\alpha^{\prime}}{2}\right)+b^{2} s^{\prime 2}} .
\end{aligned}
$$

2.

$$
\begin{aligned}
& \left|\frac{1}{t} \partial \log \left(\frac{A^{\prime}[u+t g]}{A^{\prime}[u]}\right)-\partial \frac{\partial_{u} A[g]}{A^{\prime}[u]}\right| \\
& \leq C t b^{2} \frac{\left(\sin ^{2}\left(\frac{\alpha^{\prime}}{2}\right)+b s^{\prime 2}\right)^{2}}{\left(\sin ^{2}\left(\frac{\alpha^{\prime}}{2}\right)+b^{2} s^{\prime 2}\right)^{2}}+C t b^{2} \frac{\sin ^{2}\left(\frac{\alpha^{\prime}}{2}\right)+s^{\prime 2}}{\sin ^{2}\left(\frac{\alpha^{\prime}}{2}\right)+b^{2} s^{\prime 2}} \\
& +C t b^{3} \frac{\left(\sin ^{2}\left(\frac{\alpha^{\prime}}{2}\right)+b s^{\prime 2}\right)^{3}}{\left(\sin ^{2}\left(\frac{\alpha^{\prime}}{2}\right)+b^{2} s^{\prime 2}\right)^{3}}+C t b^{3} \frac{\left(\sin ^{2}\left(\frac{\alpha^{\prime}}{2}\right)+b s^{\prime 3}\right)\left(\sin ^{2}\left(\frac{\alpha^{\prime}}{2}\right)+s^{\prime 2}\right)}{\left(\sin ^{2}\left(\frac{\alpha^{\prime}}{2}\right)+b^{2} s^{\prime 2}\right)^{2}} .
\end{aligned}
$$

3.

$$
\begin{aligned}
& \left|\frac{1}{t} \partial^{2} \log \left(\frac{A^{\prime}[u+t g]}{A^{\prime}[u]}\right)-\partial^{2} \frac{\partial_{u} A[g]}{A^{\prime}[u]}\right| \\
& \leq C b^{2} t \frac{\left(\sin ^{2}\left(\frac{\alpha^{\prime}}{2}\right)+b s^{\prime 2}\right)\left(\sin ^{2}\left(\frac{\alpha^{\prime}}{2}\right)+b\left(\left|\sin \left(\frac{\alpha^{\prime}}{2}\right)\right|+\left|s^{\prime}\right|+s^{\prime 2}\right)\right)}{\left(\sin ^{2}\left(\frac{\alpha^{\prime}}{2}\right)+b^{2} s^{\prime 2}\right)^{2}} \\
& +C b^{2} t \frac{\left(\sin ^{2}\left(\frac{\alpha^{\prime}}{2}\right)+b s^{\prime 2}\right)^{2}}{\left(\sin ^{2}\left(\frac{\alpha^{\prime}}{2}\right)+b^{2} s^{\prime 2}\right)^{2}}+C b^{3} t \frac{\left(\sin ^{2}\left(\frac{\alpha^{\prime}}{2}\right)+b s^{\prime 2}\right)^{3}}{\left(\sin ^{2}\left(\frac{\alpha^{\prime}}{2}\right)+b^{2} s^{\prime 2}\right)^{3}} \\
& +C b^{2} t \frac{\sin ^{2}\left(\frac{\alpha^{\prime}}{2}\right)+\left|\sin \left(\frac{\alpha^{\prime}}{2}\right)\right|+s^{\prime 2}+\left|s^{\prime}\right|}{\sin ^{2}\left(\frac{\alpha^{\prime}}{2}\right)+b^{2} s^{\prime 2}}+C b^{3} t \frac{\left(\sin ^{2}\left(\frac{\alpha^{\prime}}{2}\right)+b s^{\prime 2}\right)\left(\sin ^{2}\left(\frac{\alpha^{\prime}}{2}\right)+s^{\prime 2}\right)}{\left(\sin ^{2}\left(\frac{\alpha^{\prime}}{2}\right)+b^{2} s^{\prime 2}\right)^{2}}
\end{aligned}
$$




$$
\begin{aligned}
& +C b^{3} t\left(\frac{\left(\sin ^{2}\left(\frac{\alpha^{\prime}}{2}\right)+b\left(\left|\sin \left(\frac{\alpha^{\prime}}{2}\right)\right|+s^{\prime 2}+\left|s^{\prime}\right|\right)\right)}{\left(\sin ^{2}\left(\frac{\alpha^{\prime}}{2}\right)+b^{2} s^{\prime 2}\right)^{3}}+b \frac{\left(\sin ^{2}\left(\frac{\alpha^{\prime}}{2}\right)+b s^{\prime 2}\right)^{2}}{\left(\sin ^{2}\left(\frac{\alpha^{\prime}}{2}\right)+b^{2} s^{\prime 2}\right)^{4}}\right)\left(\sin ^{2}\left(\frac{\alpha^{\prime}}{2}\right)+b s^{\prime 2}\right)^{2} \\
& +C b^{3} t \frac{\left(\sin ^{2}\left(\frac{\alpha^{\prime}}{2}\right)+b\left(\left|\sin \left(\frac{\alpha^{\prime}}{2}\right)\right|+\left|s^{\prime}\right|+s^{\prime 2}\right)\right)}{\left(\sin ^{2}\left(\frac{\alpha^{\prime}}{2}\right)+b^{2} s^{\prime 2}\right)^{2}}\left(\sin ^{2}\left(\frac{\alpha^{\prime}}{2}\right)+s^{\prime 2}\right) \\
& +C b^{4} t \frac{\left(\sin ^{2}\left(\frac{\alpha}{2}\right)+b s^{\prime 2}\right)^{2}}{\left(\sin ^{2}\left(\frac{\alpha^{\prime}}{2}\right)+b^{2} s^{\prime 2}\right)^{3}}\left(\sin ^{2}\left(\frac{\alpha^{\prime}}{2}\right)+s^{\prime 2}\right)
\end{aligned}
$$

4.

$$
\begin{aligned}
& \left|\frac{1}{t} \partial^{3} \log \left(\frac{A^{\prime}[u+t g]}{A^{\prime}[u]}\right)-\partial^{3} \frac{\partial_{u} A[g]}{A^{\prime}[u]}\right| \leq \\
& C b \frac{\sin ^{2}\left(\frac{\alpha^{\prime}}{2}\right)+b s^{\prime 2}}{\left(\sin ^{2}\left(\frac{\alpha^{\prime}}{2}\right)+b^{2} s^{\prime 2}\right)^{2}} \\
& \times\left(b t \sin ^{2}\left(\frac{\alpha^{\prime}}{2}\right)\left|\partial^{3}\left(g+g^{\prime}\right)\right|+C b^{2} t \sin ^{2}\left(\frac{\alpha^{\prime}}{2}\right)\left|\partial^{3}\left(u g^{\prime}+g u^{\prime}\right)\right|\right. \\
& +b^{2} t\left(\left|\sin \left(\frac{\alpha^{\prime}}{2}\right)\right|+\left|s^{\prime}\right|\right)\left|\partial^{3}\left(u-u^{\prime}\right)\right|+b^{2} t\left(\left|\sin \left(\frac{\alpha^{\prime}}{2}\right)\right|+\left|s^{\prime}\right|\right)\left|\partial^{3}\left(g-g^{\prime}\right)\right| \\
& \left.+b^{2} t\left(\left|\sin \left(\frac{\alpha^{\prime}}{2}\right)\right|+\left|s^{\prime}\right|\right)+b^{2} t \sin ^{2}\left(\frac{\alpha^{\prime}}{2}\right)\left|\partial^{3}\left(g g^{\prime}\right)\right|\right) \\
& +C b^{2} t \frac{\sin ^{2}\left(\frac{\alpha^{\prime}}{2}\right)}{\sin ^{2}\left(\frac{\alpha^{\prime}}{2}\right)+b^{2} s^{\prime 2}}\left|\partial^{3}\left(g g^{\prime}\right)\right|+C b^{2} t \frac{\left|\sin \left(\frac{\alpha^{\prime}}{2}\right)\right|+\left|s^{\prime}\right|}{\sin ^{2}\left(\frac{\alpha^{\prime}}{2}\right)+b^{2} s^{\prime 2}}\left|\partial^{3}\left(g-g^{\prime}\right)\right| \\
& +C b^{2} t\left(\sin ^{2}\left(\frac{\alpha^{\prime}}{2}\right)+b s^{\prime 2}\right)^{2} \\
& \times\left(b \frac{\sin ^{2}\left(\frac{\alpha^{\prime}}{2}\right)}{\left(\sin ^{2}\left(\frac{\alpha^{\prime}}{2}\right)+b^{2} s^{\prime 2}\right)^{3}}\left|\partial^{3}\left(u+u^{\prime}\right)\right|+b^{2} \frac{\sin ^{2}\left(\frac{\alpha^{\prime}}{2}\right)}{\left(\sin ^{2}\left(\frac{\alpha^{\prime}}{2}\right)+b^{2} s^{\prime 2}\right)^{3}}\left|\partial^{3}\left(u u^{\prime}\right)\right|\right. \\
& \left.+C b^{2} \frac{\left|\sin \left(\frac{\alpha^{\prime}}{2}\right)\right|+\left|s^{\prime}\right|}{\left(\sin ^{2}\left(\frac{\alpha^{\prime}}{2}\right)+b^{2} s^{\prime 2}\right)^{3}}\left|\partial^{3}\left(u-u^{\prime}\right)\right|\right) \\
& +C b^{2} t\left(\sin ^{2}\left(\frac{\alpha^{\prime}}{2}\right)+s^{\prime 2}\right) \\
& \times\left(b \frac{\sin ^{2}\left(\frac{\alpha^{\prime}}{2}\right)}{\left(\sin ^{2}\left(\frac{\alpha^{\prime}}{2}\right)+b^{2} s^{\prime 2}\right)^{2}}\left|\partial^{3}\left(u+u^{\prime}\right)\right|+b^{2} \frac{\sin ^{2}\left(\frac{\alpha^{\prime}}{2}\right)}{\left(\sin ^{2}\left(\frac{\alpha^{\prime}}{2}\right)+b^{2} s^{\prime 2}\right)^{2}}\left|\partial^{3}\left(u u^{\prime}\right)\right|\right. \\
& \left.+b^{2} \frac{\left|\sin \left(\frac{\alpha^{\prime}}{2}\right)\right|+\left|s^{\prime}\right|}{\left(\sin ^{2}\left(\frac{\alpha^{\prime}}{2}\right)+b^{2} s^{\prime 2}\right)^{2}}\left|\partial^{3}\left(u-u^{\prime}\right)\right|\right) \\
& +\operatorname{KERNEL} L_{b}\left(\alpha^{\prime}, s^{\prime}\right) \text {. }
\end{aligned}
$$

where

$$
\left\|K E R N E L_{b}\right\|_{L^{1}\left(\Omega_{a}\right)} \leq C b t
$$


Proof: First we focus on the inequality 1 . We have that

$$
\begin{aligned}
\frac{d A^{\prime}[u+t g]}{d t} & =b\left(g+g^{\prime}\right) \sin ^{2}\left(\frac{\alpha^{\prime}}{2}\right)+4\left(\left(u g^{\prime}+u^{\prime} g\right) \sin ^{2}\left(\frac{\alpha^{\prime}}{2}\right)+2\left(u-u^{\prime}\right)\left(g-g^{\prime}\right)\right) b^{2} \\
& +2 t b^{2}\left(4 g g^{\prime} \sin ^{2}\left(\frac{\alpha^{\prime}}{2}\right)+\left(g-g^{\prime}\right)^{2}\right) \\
& =\partial_{u} A^{\prime}\left[g^{\prime}\right]+2 t b^{2}\left(4 g g^{\prime} \sin ^{2}\left(\frac{\alpha^{\prime}}{2}\right)+\left(g-g^{\prime}\right)^{2}\right) .
\end{aligned}
$$

In addition

$$
\log \left(\frac{A^{\prime}[u+t g]}{A^{\prime}[u]}\right)=\int_{0}^{1} \frac{d}{d \mu} \log \left(A^{\prime}[u+t \mu g]\right) d \mu
$$

and therefore

$$
\begin{aligned}
& \frac{1}{t} \log \left(\frac{A^{\prime}[u+t g]}{A^{\prime}[u]}\right)-\frac{\partial_{u} A^{\prime}[g]}{A^{\prime}[u]} \\
= & \int_{0}^{1}\left(\frac{\partial_{u} A^{\prime}[g]+2 b^{2} t \mu\left(4 g g^{\prime} \sin ^{2}\left(\frac{\alpha^{\prime}}{2}\right)+\left(g-g^{\prime}\right)^{2}\right)}{A^{\prime}[u+t \mu g]}-\frac{\partial_{u} A^{\prime}[g]}{A^{\prime}[u]}\right) d \mu \\
= & \frac{\partial_{u} A^{\prime}[g]}{A^{\prime}[u]} \int_{0}^{1} \frac{A^{\prime}[u]-A^{\prime}[u+t \mu g]}{A^{\prime}[u+t \mu g]} d \mu+2 b^{2} t\left(4 g g^{\prime} \sin ^{2}\left(\frac{\alpha^{\prime}}{2}\right)+\left(g-g^{\prime}\right)^{2}\right) \int_{0}^{1} \frac{\mu}{A^{\prime}[u+\mu t g]} d \mu .
\end{aligned}
$$

By using lemma B.20-1 and B.20-3 we can conclude

$$
\left|\frac{A^{\prime}[u+t \mu g]-A^{\prime}[u]}{A^{\prime}[u+t \mu g]}\right| \leq C \mu t \frac{b \sin ^{2}\left(\frac{\alpha^{\prime}}{2}\right)+b^{2} s^{\prime 2}}{\sin ^{2}\left(\frac{\alpha^{\prime}}{2}\right)+b^{2} s^{\prime 2}}+C \mu^{2} t^{2} b^{2} \frac{\sin ^{2}\left(\frac{\alpha^{\prime}}{2}\right)+s^{\prime 2}}{\sin ^{2}\left(\frac{\alpha^{\prime}}{2}\right)+b^{2} s^{\prime 2}}
$$

and therefore

$$
\left|\frac{\partial_{u} A^{\prime}[g]}{A^{\prime}[u]} \int_{0}^{1} \frac{A^{\prime}[u]-A^{\prime}[u+t \mu g]}{A^{\prime}[u+t \mu g]} d \mu\right| \leq C \frac{b \sin ^{2}\left(\frac{\alpha^{\prime}}{2}\right)+b^{2} s^{\prime 2}}{\sin ^{2}\left(\frac{\alpha^{\prime}}{2}\right)+b^{2} s^{\prime 2}}\left(t \frac{b \sin ^{2}\left(\frac{\alpha^{\prime}}{2}\right)+b^{2} s^{\prime 2}}{\sin ^{2}\left(\frac{\alpha^{\prime}}{2}\right)+b^{2} s^{\prime 2}}+t^{2} b^{2} \frac{\sin ^{2}\left(\frac{\alpha^{\prime}}{2}\right)+s^{\prime 2}}{\sin ^{2}\left(\frac{\alpha^{\prime}}{2}\right)+b^{2} s^{\prime 2}}\right) .
$$

In addition

$$
\left|2 b^{2} t\left(4 g g^{\prime} \sin ^{2}\left(\frac{\alpha^{\prime}}{2}\right)+\left(g-g^{\prime}\right)^{2}\right) \int_{0}^{1} \frac{\mu}{A^{\prime}[u+t \mu g]} d \mu\right| \leq C b^{2} t \frac{\sin ^{2}\left(\frac{\alpha^{\prime}}{2}\right)+s^{\prime 2}}{\sin ^{2}\left(\frac{\alpha^{\prime}}{2}\right)+b^{2} s^{\prime 2}}
$$

Then by using lemma B.2 we can conclude the inequality 1 of the lemma.

To prove the inequality 2 we take one derivative

$$
\begin{aligned}
& \partial \frac{1}{t} \log \left(\frac{A^{\prime}[u+t g]}{A^{\prime}[u]}\right)-\partial \frac{\partial_{u} A^{\prime}[g]}{A^{\prime}[u]}=\frac{1}{t}\left(\frac{\partial A^{\prime}[u+t g]}{A^{\prime}[u+t g]}-\frac{\partial A^{\prime}[u]}{A^{\prime}[u]}\right)-\partial \frac{\partial_{u} A^{\prime}[g]}{A^{\prime}[u]} \\
& =\frac{1}{t}\left(\frac{\partial A^{\prime}[u+t g]-\partial A[u]}{A^{\prime}[u+t g]}\right)-\frac{\partial \partial_{u} A^{\prime}[u]}{A^{\prime}[u]}+\frac{1}{t} \partial A[u]\left(\frac{1}{A^{\prime}[u+t g]}-\frac{1}{A^{\prime}[u]}\right)+\frac{\partial A^{\prime}[u]}{A^{\prime}[u]^{2}} \partial_{u} A^{\prime}[g] \\
& =\frac{1}{t}\left(\partial A^{\prime}[u+t g]-\partial A^{\prime}[u]\right)\left(\frac{1}{A^{\prime}[u+t g]}-\frac{1}{A^{\prime}[u]}\right)+\frac{1}{t} \frac{\partial A^{\prime}[u+t g]-\partial A[u]-t \partial \partial_{u} A^{\prime}[g]}{A^{\prime}[u]}
\end{aligned}
$$




$$
\begin{aligned}
& +\frac{\partial A^{\prime}[u]}{t}\left(\frac{1}{A^{\prime}[u+t g]}-\frac{1}{A^{\prime}[u]}+t \frac{\partial_{u} A^{\prime}[g]}{A^{\prime}[u]^{2}}\right) \\
& =\frac{1}{t}\left(\partial A^{\prime}[u+t g]-\partial A^{\prime}[u]\right)\left(A^{\prime}[u]-A^{\prime}[u+t g]\right) \frac{1}{A^{\prime}[u+t g] A^{\prime}[u]} \\
& +\frac{1}{t} \frac{\partial A^{\prime}[u+t g]-\partial A[u]-t \partial \partial_{u} A^{\prime}[g]}{A^{\prime}[u]} \\
& +\frac{1}{t} \frac{\partial A^{\prime}[u]}{A^{\prime}[u]^{2} A^{\prime}[u+t g]}\left(A^{\prime}[u]-A^{\prime}[u+t g]\right)^{2} \\
& +\frac{1}{t} \frac{\partial A^{\prime}[u]}{A^{\prime}[u]^{2}}\left(A^{\prime}[u]-A^{\prime}[u+t g]+t \partial_{u} A^{\prime}[g]\right) \\
& =J_{1}+J_{2}+J_{3}+J_{4} .
\end{aligned}
$$

And we can apply B.20 and B.2 to obtain

$$
\begin{aligned}
& \left|J_{1}\right|=\left|\frac{1}{t} \frac{A^{\prime}[u+t g]-A^{\prime}[u]}{A^{\prime}[u+t g] A^{\prime}[u]} \partial\left(A^{\prime}[u+t g]-A^{\prime}[u]\right)\right| \leq C t b^{2} \frac{\left(\sin ^{2}\left(\frac{\alpha^{\prime}}{2}\right)+b s^{\prime 2}\right)^{2}}{\left(\sin ^{2}\left(\frac{\alpha^{\prime}}{2}\right)+b^{2} s^{\prime 2}\right)^{2}} \\
& \left|J_{2}\right|=\left|\frac{1}{t} \frac{\partial A^{\prime}[u+t g]-\partial A^{\prime}[u]-t \partial \partial_{u} A^{\prime}[g]}{A^{\prime}[u]}\right| \leq C t b^{2} \frac{\sin ^{2}\left(\frac{\alpha^{\prime}}{2}\right)+s^{\prime 2}}{\sin ^{2}\left(\frac{\alpha^{\prime}}{2}\right)+b^{2} s^{\prime 2}} \\
& \left|J_{3}\right|=\left|\frac{\partial A^{\prime}[u]}{t} \frac{\left(A^{\prime}[u+t g]-A^{\prime}[u]\right)^{2}}{A^{\prime}[u]^{2} A^{\prime}[u+t g]}\right| \leq C t b^{3} \frac{\left(\sin ^{2}\left(\frac{\alpha^{\prime}}{2}\right)+b s^{\prime 2}\right)^{3}}{\left(\sin ^{2}\left(\frac{\alpha^{\prime}}{2}\right)+b^{2} s^{\prime 2}\right)^{3}} \\
& \left|J_{4}\right|=\left|\frac{\partial A^{\prime}[u]}{t} \frac{A^{\prime}[u]-A^{\prime}[u+t g]+t \partial_{u} A^{\prime}[g]}{A^{\prime}[u]^{2}}\right| \\
& \leq C t b^{3} \frac{\left(\sin ^{2}\left(\frac{\alpha^{\prime}}{2}\right)+b s^{\prime 3}\right)\left(\sin ^{2}\left(\frac{\alpha^{\prime}}{2}\right)+s^{\prime 2}\right)}{\left(\sin ^{2}\left(\frac{\alpha^{\prime}}{2}\right)+b^{2} s^{\prime 2}\right)^{2}} .
\end{aligned}
$$

In order to prove the inequality 3 we compute two derivatives to obtain

$$
\begin{aligned}
& \partial J_{1}=\frac{1}{t} \frac{\partial^{2}\left(A^{\prime}[u+t g]-A^{\prime}[u]\right)\left(A^{\prime}[u+t g]-A^{\prime}[u]\right)}{A^{\prime}[u+t g] A^{\prime}[u]}+\frac{1}{t} \frac{\left(\partial A^{\prime}[u+t g]-\partial A^{\prime}[u]\right)^{2}}{A^{\prime}[u+t g] A^{\prime}[u]} \\
& -\frac{1}{t}\left(\partial A^{\prime}[u+t g]-\partial A^{\prime}[u]\right)\left(A^{\prime}[u+t g]-A^{\prime}[u]\right) \partial \frac{1}{A^{\prime}[u+t g] A^{\prime}[u]} \\
& \equiv J_{11}+J_{12}+J_{13} .
\end{aligned}
$$

Since

$$
\partial \frac{1}{A^{\prime}[u+t g] A^{\prime}[u]}=\frac{1}{A^{\prime}[u+t g] A^{\prime}[u]}\left(\frac{\partial A^{\prime}[u+t g]}{A^{\prime}[u+t g]}+\frac{\partial A^{\prime}[u]}{A^{\prime}[u]}\right)
$$

and therefore

$$
\left|\partial \frac{1}{A^{\prime}[u+t g] A^{\prime}[u]}\right| \leq C b \frac{\sin ^{2}\left(\frac{\alpha^{\prime}}{2}\right)+b s^{\prime 2}}{\left(\sin ^{2}\left(\frac{\alpha^{\prime}}{2}\right)+b^{2} s^{\prime 2}\right)^{3}}
$$


we have that

$$
\begin{aligned}
& \left|J_{11}\right| \leq C b^{2} t \frac{\left(\sin ^{2}\left(\frac{\alpha^{\prime}}{2}\right)+b s^{\prime 2}\right)\left(\sin ^{2}\left(\frac{\alpha^{\prime}}{2}\right)+b\left(\left|\sin \left(\frac{\alpha^{\prime}}{2}\right)\right|+\left|s^{\prime}\right|+s^{\prime 2}\right)\right)}{\left(\sin ^{2}\left(\frac{\alpha^{\prime}}{2}\right)+b^{2} s^{\prime 2}\right)^{2}} \\
& \left|J_{12}\right| \leq C b^{2} t \frac{\left(\sin ^{2}\left(\frac{\alpha^{\prime}}{2}\right)+b s^{\prime 2}\right)^{2}}{\left(\sin ^{2}\left(\frac{\alpha^{\prime}}{2}\right)+b^{2} s^{\prime 2}\right)^{2}} \\
& \left|J_{13}\right| \leq C b^{3} t \frac{\left(\sin ^{2}\left(\frac{\alpha^{\prime}}{2}\right)+b s^{\prime 2}\right)^{3}}{\left(\sin ^{2}\left(\frac{\alpha^{\prime}}{2}\right)+b^{2} s^{\prime 2}\right)^{3}} .
\end{aligned}
$$

In addition

$$
\begin{aligned}
& \partial J_{2}=\frac{1}{t} \frac{\partial^{2} A^{\prime}[u+t g]-\partial^{2} A^{\prime}[u]-t \partial^{2} \partial_{u} A^{\prime}[g]}{A^{\prime}[u]}-\frac{\partial A^{\prime}[u]}{t} \frac{\partial A^{\prime}[u+t g]-\partial A^{\prime}[u]-t \partial \partial_{u} A^{\prime}[g]}{A^{\prime}[u]^{2}} \\
& \equiv J_{21}+J_{22} .
\end{aligned}
$$

And we can estimate

$$
\begin{gathered}
\left|J_{21}\right| \leq C b^{2} t \frac{\sin ^{2}\left(\frac{\alpha^{\prime}}{2}\right)+\left|\sin \left(\frac{\alpha^{\prime}}{2}\right)\right|+s^{\prime 2}+\left|s^{\prime}\right|}{\sin ^{2}\left(\frac{\alpha^{\prime}}{2}\right)+b^{2} s^{\prime 2}} \\
\left|J_{22}\right| \leq C b^{3} t \frac{\left(\sin ^{2}\left(\frac{\alpha^{\prime}}{2}\right)+b s^{\prime 2}\right)\left(\sin ^{2}\left(\frac{\alpha^{\prime}}{2}\right)+s^{\prime 2}\right)}{\left(\sin ^{2}\left(\frac{\alpha^{\prime}}{2}\right)+b^{2} s^{\prime 2}\right)^{2}} \\
\partial J_{3}=\left(A^{\prime}[u+t g]-A^{\prime}[u]\right)^{2} \partial \frac{\partial A^{\prime}[u]}{A^{\prime}[u+t g] A^{\prime}[u]^{2}}+2 \frac{\partial A^{\prime}[u]}{t} \frac{\left(A^{\prime}[u]-A^{\prime}[u+t g]\right)\left(\partial A^{\prime}[u]-\partial A^{\prime}[u+t g]\right)}{A^{\prime}[u]^{2} A^{\prime}[u+t g]} \\
\equiv J_{32}+J_{31}
\end{gathered}
$$

Since

$$
\partial \frac{\partial A^{\prime}[u]}{A^{\prime}[u+t g] A^{\prime}[u]^{2}}=\frac{\partial^{2} A^{\prime}[u]}{A^{\prime}[u+t g] A^{\prime}[u]^{2}}-\frac{\partial A^{\prime}[u] \partial A^{\prime}[u+t g]}{A^{\prime}[u+t g]^{2} A^{\prime}[u]^{2}}-2 \frac{\partial A^{\prime}[u]^{2}}{A^{\prime}[u+t g] A^{\prime}[u]^{3}}
$$

we can estimate that

$$
\begin{aligned}
& \left|\partial \frac{\partial A^{\prime}[u]}{A^{\prime}[u+t g] A^{\prime}[u]^{2}}\right| \leq C b \frac{\left(\sin ^{2}\left(\frac{\alpha^{\prime}}{2}\right)+b\left(\left|\sin \left(\frac{\alpha^{\prime}}{2}\right)\right|+s^{\prime 2}+\left|s^{\prime}\right|\right)\right)}{\left(\sin ^{2}\left(\frac{\alpha^{\prime}}{2}\right)+b^{2} s^{\prime 2}\right)^{3}} \\
& +C b^{2} \frac{\left(\sin ^{2}\left(\frac{\alpha^{\prime}}{2}\right)+b s^{\prime 2}\right)^{2}}{\left(\sin ^{2}\left(\frac{\alpha^{\prime}}{2}\right)+b^{2} s^{\prime 2}\right)^{4}}
\end{aligned}
$$

And we have that

$$
\left|J_{31}\right| \leq C b^{3} t \frac{\left(\sin ^{2}\left(\frac{\alpha^{\prime}}{2}\right)+b^{\prime 2}\right)^{3}}{\left(\sin ^{2}\left(\frac{\alpha^{\prime}}{2}\right)+b^{2} s^{\prime 2}\right)^{3}}
$$




$$
\begin{aligned}
& \left|J_{32}\right| \leq C b^{3} t\left(\frac{\left(\sin ^{2}\left(\frac{\alpha^{\prime}}{2}\right)+b\left(\left|\sin \left(\frac{\alpha^{\prime}}{2}\right)\right|+s^{\prime 2}+\left|s^{\prime}\right|\right)\right)}{\left(\sin ^{2}\left(\frac{\alpha^{\prime}}{2}\right)+b^{2} s^{\prime 2}\right)^{3}}+\frac{\left(\sin ^{2}\left(\frac{\alpha^{\prime}}{2}\right)+b s^{\prime 2}\right)^{2}}{\left(\sin ^{2}\left(\frac{\alpha^{\prime}}{2}\right)+b^{2} s^{\prime 2}\right)^{4}}\right)\left(\sin ^{2}\left(\frac{\alpha^{\prime}}{2}\right)+b s^{\prime 2}\right)^{2} \\
& \partial J_{4}=\frac{\partial A^{\prime}[u]}{t} \frac{\partial A^{\prime}[u]-\partial A^{\prime}[u+t g]+t \partial \partial_{u} A^{\prime}[g]}{A^{\prime}[u]^{2}}+\partial\left(\frac{\partial A^{\prime}[u]}{A^{\prime}[u]^{2}}\right) \frac{1}{t}\left(A^{\prime}[u]-A^{\prime}[u+t g]+t \partial_{u} A^{\prime}[g]\right) \\
& \equiv J_{41}+J_{42},
\end{aligned}
$$

where $J_{41}$ is the same than $J_{22}$. Since

$$
\partial\left(\frac{\partial A^{\prime}[u]}{A^{\prime}[u]^{2}}\right)=\frac{\partial^{2} A^{\prime}[u]}{A^{\prime}[u]^{2}}-2 \frac{\left(\partial A^{\prime}[u]\right)^{2}}{A^{\prime}[u]^{3}}
$$

and then

$$
\begin{gathered}
\left|\partial\left(\frac{\partial A^{\prime}[a]}{A^{\prime}[a]^{2}}\right)\right| \leq C b \frac{\left(\sin ^{2}\left(\frac{\alpha^{\prime}}{2}\right)+b\left(\left|\sin \left(\frac{\alpha^{\prime}}{2}\right)\right|+\left|s^{\prime}\right|+s^{\prime 2}\right)\right)}{\left(\sin ^{2}\left(\frac{\alpha^{\prime}}{2}\right)+b^{2} s^{\prime 2}\right)^{2}}+C b^{2} \frac{\left(\sin ^{2}\left(\frac{\alpha}{2}\right)+b s^{\prime 2}\right)^{2}}{\left(\sin ^{2}\left(\frac{\alpha^{\prime}}{2}\right)+b^{2} s^{\prime 2}\right)^{3}}, \\
\left|J_{42}\right| \leq C b^{3} t \frac{\left(\sin ^{2}\left(\frac{\alpha^{\prime}}{2}\right)+b\left(\left|\sin \left(\frac{\alpha^{\prime}}{2}\right)\right|+\left|s^{\prime}\right|+s^{\prime 2}\right)\right)}{\left(\sin ^{2}\left(\frac{\alpha^{\prime}}{2}\right)+b^{2} s^{\prime 2}\right)^{2}}\left(\sin ^{2}\left(\frac{\alpha^{\prime}}{2}\right)+s^{\prime 2}\right) \\
+C b^{4} t \frac{\left(\sin ^{2}\left(\frac{\alpha}{2}\right)+b s^{\prime 2}\right)^{2}}{\left(\sin ^{2}\left(\frac{\alpha^{\prime}}{2}\right)+b^{2} s^{\prime 2}\right)^{3}}\left(\sin ^{2}\left(\frac{\alpha^{\prime}}{2}\right)+s^{\prime 2}\right)
\end{gathered}
$$

Finally we compute 3 derivatives. We have to differentiate $J_{11}, J_{12}, J_{13}, J_{21}, J_{22}, J_{31}, J_{32}$ and $J_{42}$.

For $J_{11}$ we have that

$$
\begin{aligned}
& \partial J_{11}=\frac{1}{t} \frac{\left(\partial^{3} A^{\prime}[u+t g]-\partial^{3} A^{\prime}[u]\right)\left(A^{\prime}[u+t g]-A^{\prime}[u]\right)}{A^{\prime}[u+t g] A^{\prime}[u]}+\frac{1}{t} \frac{\left(\partial^{2} A^{\prime}[u+t g]-\partial^{2} A^{\prime}[u]\right)\left(\partial A^{\prime}[u+t g]-\partial A^{\prime}[u]\right)}{A^{\prime}[u+t g] A^{\prime}[u]} \\
& -\frac{1}{t}\left(\partial^{2} A^{\prime}[u+t g]-\partial^{2} A^{\prime}[u]\right)\left(A^{\prime}[u+t g]-A^{\prime}[u]\right) \partial\left(\frac{1}{A^{\prime}[u+t g] A^{\prime}[u]}\right) \\
& \equiv J_{111}+J_{112}+J_{113} .
\end{aligned}
$$

In order to bound this terms we notice that

$$
\begin{aligned}
& \partial^{3} A^{\prime}[u+t g]-\partial^{3} A^{\prime}[u]=4 b t \partial^{3}\left(g+g^{\prime}\right) \sin ^{2}\left(\frac{\alpha^{\prime}}{2}\right) \\
& +b^{2} t\left(4 \partial^{3}\left(u g^{\prime}+g u^{\prime}\right) \sin ^{2}\left(\frac{\alpha^{\prime}}{2}\right)+2\left(g-g^{\prime}\right) \partial^{3}\left(u-u^{\prime}\right)+2\left(u-u^{\prime}\right) \partial^{3}\left(g-g^{\prime}\right)\right) \\
& +b^{2} t\left(6 \partial\left(u-u^{\prime}\right) \partial^{2}\left(g-g^{\prime}\right)+6 \partial\left(g-g^{\prime}\right) \partial^{2}\left(u-u^{\prime}\right)\right) \\
& +b^{2} t^{2}\left(4 \partial^{3}\left(g g^{\prime}\right) \sin ^{2}\left(\frac{\alpha^{\prime}}{2}\right)+4\left(g-g^{\prime}\right) \partial^{3}\left(g-g^{\prime}\right)+12 \partial\left(g-g^{\prime}\right) \partial^{2}\left(g-g^{\prime}\right)\right)
\end{aligned}
$$

Thus

$$
\left|\partial^{3} A^{\prime}[u+t g]-\partial^{3} A^{\prime}[u]\right| \leq C b t \sin ^{2}\left(\frac{\alpha^{\prime}}{2}\right)\left|\partial^{3}\left(g+g^{\prime}\right)\right|+C b^{2} t \sin ^{2}\left(\frac{\alpha^{\prime}}{2}\right)\left|\partial^{3}\left(u g^{\prime}+g u^{\prime}\right)\right|
$$




$$
\begin{aligned}
& +C b^{2} t\left(\left|\sin \left(\frac{\alpha^{\prime}}{2}\right)\right|+\left|s^{\prime}\right|\right)\left|\partial^{3}\left(u-u^{\prime}\right)\right|+C b^{2} t\left(\left|\sin \left(\frac{\alpha^{\prime}}{2}\right)\right|+\left|s^{\prime}\right|\right)\left|\partial^{3}\left(g-g^{\prime}\right)\right| \\
& +C b^{2} t\left(\left|\sin \left(\frac{\alpha^{\prime}}{2}\right)\right|+\left|s^{\prime}\right|\right)+C b^{2} t^{2} \sin ^{2}\left(\frac{\alpha^{\prime}}{2}\right)\left|\partial^{3}\left(g g^{\prime}\right)\right|
\end{aligned}
$$

And using lemma B.20 and B.7 we obtain

$$
\begin{aligned}
& \left|J_{111}\right| \leq C b \frac{\sin ^{2}\left(\frac{\alpha^{\prime}}{2}\right)+b s^{\prime 2}}{\left(\sin ^{2}\left(\frac{\alpha^{\prime}}{2}\right)+b^{2} s^{\prime 2}\right)^{2}} \\
& \times\left(b t \sin ^{2}\left(\frac{\alpha^{\prime}}{2}\right)\left|\partial^{3}\left(g+g^{\prime}\right)\right|+C b^{2} t \sin ^{2}\left(\frac{\alpha^{\prime}}{2}\right)\left|\partial^{3}\left(u g^{\prime}+g u^{\prime}\right)\right|\right. \\
& +b^{2} t\left(\left|\sin \left(\frac{\alpha^{\prime}}{2}\right)\right|+\left|s^{\prime}\right|\right)\left|\partial^{3}\left(u-u^{\prime}\right)\right|+b^{2} t\left(\left|\sin \left(\frac{\alpha^{\prime}}{2}\right)\right|+\left|s^{\prime}\right|\right)\left|\partial^{3}\left(g-g^{\prime}\right)\right| \\
& \left.+b^{2} t\left(\left|\sin \left(\frac{\alpha^{\prime}}{2}\right)\right|+\left|s^{\prime}\right|\right)+b^{2} t^{2} \sin ^{2}\left(\frac{\alpha^{\prime}}{2}\right)\left|\partial^{3}\left(g g^{\prime}\right)\right|\right) \\
& \left|J_{112}\right| \leq C b^{2} t \frac{\left(\sin ^{2}\left(\frac{\alpha^{\prime}}{2}\right)+b s^{\prime 2}\right)\left(\sin ^{2}\left(\frac{\alpha^{\prime}}{2}\right)+b\left(\left|\sin \left(\frac{\alpha^{\prime}}{2}\right)\right|+\left|s^{\prime}\right|+s^{\prime 2}\right)\right)}{\left(\sin ^{2}\left(\frac{\alpha^{\prime}}{2}\right)+b^{2} s^{\prime 2}\right)^{2}} \\
& \left|J_{113}\right| \leq C b^{3} t \frac{\left(\sin ^{2}\left(\frac{\alpha^{\prime}}{2}\right)+b s^{\prime 2}\right)^{2}\left(\sin ^{2}\left(\frac{\alpha^{\prime}}{2}\right)+b\left(\left|\sin \left(\frac{\alpha^{\prime}}{2}\right)\right|+\left|s^{\prime}\right|+s^{\prime 2}\right)\right)}{\left.\left(\sin ^{2}\right)+b^{2} s^{\prime 2}\right)^{3}}
\end{aligned}
$$

For $J_{12}$

$$
\begin{aligned}
& \partial J_{12}=\frac{2}{t} \frac{\left(\partial A^{\prime}[u+t g]-\partial A^{\prime}[u]\right)\left(\partial^{2} A^{\prime}[u+t g]-\partial^{2} A^{\prime}[u]\right)}{A^{\prime}[u+t g] A^{\prime}[u]}-\frac{1}{t}\left(\partial A^{\prime}[u+t g]-\partial A^{\prime}[u]\right)^{2} \partial \frac{1}{A^{\prime}[u+t g] A^{\prime}[u]} \\
& \equiv J_{121}+J_{122},
\end{aligned}
$$

where $J_{121}$ is equivalent to $J_{112}$. Then we just need to bound $J_{122}$. By using lemma B.20 and B.7 we have that

$$
\left|J_{122}\right| \leq C b^{3} t \frac{\left(\sin ^{2}\left(\frac{\alpha^{\prime}}{2}\right)+b s^{\prime 2}\right)^{3}}{\left(\sin ^{2}\left(\frac{\alpha^{\prime}}{2}\right)+b^{2} s^{\prime 2}\right)^{3}}
$$

For $J_{13}$

$$
\begin{aligned}
& \partial J_{13}=\frac{1}{t}\left(\partial^{2} A^{\prime}[u+t g]-\partial^{2} A^{\prime}[u]\right)\left(A^{\prime}[u+t g]-A^{\prime}[u]\right) \partial \frac{1}{A^{\prime}[u+t g] A^{\prime}[u]} \\
& +\frac{1}{t}\left(\partial A^{\prime}[u+t g]-\partial A^{\prime}[u]\right)^{2} \partial \frac{1}{A^{\prime}[u+t g] A^{\prime}[u]} \\
& +\frac{1}{t}\left(\partial A^{\prime}[u+t g]-\partial A^{\prime}[u]\right)\left(A^{\prime}[u+t g]-A^{\prime}[u]\right) \partial^{2} \frac{1}{A^{\prime}[u+t g] A^{\prime}[u]} \\
& \equiv J_{131}+J_{132}+J_{133},
\end{aligned}
$$

where $J_{131}$ is equivalent to $J_{113}$ and $J_{132}$ is equivalent to $J_{122}$. Then we just have to bound $J_{133}$. 
Since

$$
\begin{aligned}
& \partial^{2} \frac{1}{A^{\prime}[u+t g] A^{\prime}[u]}=-\partial \frac{1}{A^{\prime}[u+t g] A^{\prime}[u]}\left(\frac{\partial A^{\prime}[u+t g]}{A^{\prime}[u+t g]}+\frac{\partial A^{\prime}[u]}{A^{\prime}[u]}\right) \\
& -\frac{1}{A^{\prime}[u+t g] A^{\prime}[u]}\left(\partial \frac{\partial A^{\prime}[u+t g]}{A^{\prime}[u+t g]}+\partial \frac{\partial A^{\prime}[u+t g]}{A^{\prime}[u+t g]}\right)
\end{aligned}
$$

Then using (B.1), (B.2), (B.7) and lemma B.1 we obtain

$$
\begin{aligned}
& \left|\partial^{2} \frac{1}{A^{\prime}[u+t g] A^{\prime}[u]}\right| \\
& \leq C b^{2} \frac{\left(\sin ^{2}\left(\frac{\alpha^{\prime}}{2}\right)+b s^{\prime 2}\right)^{2}}{\left(\sin ^{2}\left(\frac{\alpha^{\prime}}{2}\right)+b^{2} s^{\prime 2}\right)^{4}}+C b \frac{\sin ^{2}\left(\frac{\alpha^{\prime}}{2}\right)+b\left(\left|\sin \left(\frac{\alpha^{\prime}}{2}\right)\right|+\left|s^{\prime}\right|+s^{\prime 2}\right)}{\left(\sin ^{2}\left(\frac{\alpha^{\prime}}{2}\right)+b^{2} s^{\prime 2}\right)^{3}}+C b^{2} \frac{\left(\sin ^{2}\left(\frac{\alpha^{\prime}}{2}\right)+b s^{\prime 2}\right)^{2}}{\left(\sin ^{2}\left(\frac{\alpha^{\prime}}{2}\right)+b^{2} s^{\prime 2}\right)^{2}}
\end{aligned}
$$

and thanks to lemma B.20

$$
\begin{aligned}
& \left|J_{133}\right| \leq C b^{2} t\left(\sin ^{2}\left(\frac{\alpha^{\prime}}{2}\right)+b s^{\prime 2}\right)^{2} \\
& \times\left(b^{2} \frac{\left(\sin ^{2}\left(\frac{\alpha^{\prime}}{2}\right)+b s^{\prime 2}\right)^{2}}{\left(\sin ^{2}\left(\frac{\alpha^{\prime}}{2}\right)+b^{2} s^{\prime 2}\right)^{4}}+b \frac{\sin ^{2}\left(\frac{\alpha^{\prime}}{2}\right)+b\left(\left|\sin \left(\frac{\alpha^{\prime}}{2}\right)\right|+\left|s^{\prime}\right|+s^{\prime 2}\right)}{\left(\sin ^{2}\left(\frac{\alpha^{\prime}}{2}\right)+b^{2} s^{\prime 2}\right)^{3}}+b^{2} \frac{\left(\sin ^{2}\left(\frac{\alpha^{\prime}}{2}\right)+b s^{\prime 2}\right)^{2}}{\left(\sin ^{2}\left(\frac{\alpha^{\prime}}{2}\right)+b^{2} s^{\prime 2}\right)^{2}}\right) \\
& \partial J_{21}=\frac{1}{t} \frac{\partial^{3} A^{\prime}[u+t g]-\partial^{3} A^{\prime}[u]-t \partial^{3} \partial_{u} A[g]}{A^{\prime}[u]} \\
& +\frac{1}{t}\left(\partial^{2} A^{\prime}[u+t g]-\partial^{2} A^{\prime}[u]-t \partial^{2} \partial_{u} A^{\prime}[g]\right) \partial \frac{1}{A^{\prime}[u]} \\
& \equiv J_{211}^{*}+J_{212} .
\end{aligned}
$$

We can estimate by using lemma B.20

$$
\begin{aligned}
& \left|J_{211}\right| \leq C b^{2} t \frac{\sin ^{2}\left(\frac{\alpha^{\prime}}{2}\right)}{\sin ^{2}\left(\frac{\alpha^{\prime}}{2}\right)+b^{2} s^{\prime 2}}\left|\partial^{3}\left(g g^{\prime}\right)\right|+C b^{2} t \frac{\left|\sin \left(\frac{\alpha^{\prime}}{2}\right)\right|+\left|s^{\prime}\right|}{\sin ^{2}\left(\frac{\alpha^{\prime}}{2}\right)+b^{2} s^{\prime 2}}+C b^{2} t \frac{\left|\sin \left(\frac{\alpha^{\prime}}{2}\right)\right|+\left|s^{\prime}\right|}{\sin ^{2}\left(\frac{\alpha^{\prime}}{2}\right)+b^{2} s^{\prime 2}}\left|\partial^{3}\left(g-g^{\prime}\right)\right| \\
& \left|J_{212}\right| \leq C t b^{3}\left(\sin ^{2}\left(\frac{\alpha^{\prime}}{2}\right)+\left|\sin \left(\frac{\alpha^{\prime}}{2}\right)\right|+s^{\prime 2}+\left|s^{\prime}\right|\right) \frac{\sin ^{2}\left(\frac{\alpha^{\prime}}{2}\right)+b s^{\prime 2}}{\left(\sin ^{2}\left(\frac{\alpha^{\prime}}{2}\right)+b^{2} s^{\prime 2}\right)^{2}}
\end{aligned}
$$

For $J_{22}$ we have that

$$
\begin{aligned}
& \partial J_{22}=\frac{1}{t} \partial^{2} \frac{1}{A^{\prime}[u]}\left(\partial A^{\prime}[u+t g]-\partial A^{\prime}[u]-t \partial \partial_{u} A^{\prime}[g]\right)+\frac{1}{t} \partial \frac{1}{A^{\prime}[u]}\left(\partial^{2} A^{\prime}[u+t g]-\partial^{2} A^{\prime}[u]-t \partial^{2} \partial_{u} A^{\prime}[g]\right) \\
& \equiv J_{221}+J_{222},
\end{aligned}
$$

where $J_{222}$ is equivalent to $J_{212}$. Then we have to bound $J_{221}$. From B.9 and lemma B.20 we have that

$$
\left|J_{221}\right| \leq C t b^{2}\left(\sin ^{2}\left(\frac{\alpha^{\prime}}{2}\right)+b s^{\prime 2}\right)
$$




$$
\times\left(b \frac{\left(\sin ^{2}\left(\frac{\alpha^{\prime}}{2}\right)+b\left(\left|\sin \left(\frac{\alpha^{\prime}}{2}\right)\right|+\left|s^{\prime}\right|+s^{\prime 2}\right)\right)}{\left(\sin ^{2}\left(\frac{\alpha^{\prime}}{2}\right)+b^{2} s^{\prime 2}\right)^{2}}+b^{2} \frac{\left(\sin ^{2}\left(\frac{\alpha}{2}\right)+b s^{\prime 2}\right)^{2}}{\left(\sin ^{2}\left(\frac{\alpha^{\prime}}{2}\right)+b^{2} s^{\prime 2}\right)^{3}}\right)
$$

For $J_{31}$ we have that

$$
\begin{aligned}
& \partial J_{31}=\frac{2}{t} \partial\left(\frac{\partial A^{\prime}[u]}{A^{\prime}[u]^{2} A^{\prime}[u+t g]}\right)\left(A^{\prime}[u]-A^{\prime}[u+t g]\right)\left(\partial A^{\prime}[u]-\partial A^{\prime}[u+t g]\right) \\
& +\frac{1}{t} \partial^{2}\left(\frac{\partial A^{\prime}[u]}{A^{\prime}[u]^{2} A^{\prime}[u+t g]}\right)\left(A^{\prime}[u]-A^{\prime}[u+t g]\right)^{2} \\
& \equiv J_{311}+J_{312} .
\end{aligned}
$$

We notice that

$$
\begin{aligned}
& \partial^{2} \frac{\partial A^{\prime}[u]}{A^{\prime}[u]^{2} A^{\prime}[u+t g]}=\frac{\partial^{3} A^{\prime}[u]}{A^{\prime}[u]^{2} A^{\prime}[u+t g]}-6 \frac{\partial^{2} A^{\prime}[u] \partial A^{\prime}[u]}{A^{\prime}[u]^{3} A^{\prime}[u+t g]}-2 \frac{\partial^{2} A^{\prime}[u] \partial A^{\prime}[u+t g]}{A^{\prime}[u]^{2} A^{\prime}[u+t g]}+6 \frac{\partial A^{\prime}[u]^{3}}{A^{\prime}[u]^{3} A^{\prime}[u+t g]} \\
& +4 \frac{\partial A^{\prime}[u]^{2} \partial A^{\prime}[u+t g]}{A^{\prime}[u]^{3} A^{\prime}[u+t g]^{2}}-\frac{\partial^{2} A^{\prime}[u+t g] \partial A^{\prime}[u]}{A^{\prime}[u]^{2} A^{\prime}[u+t g]^{2}}+2 \frac{\partial A^{\prime}[u] \partial A^{\prime}[u+t g]^{2}}{A^{\prime}[u]^{2} A^{\prime}[u+t g]^{3}}
\end{aligned}
$$

By using lemma B.1 we obtain that

$$
\begin{aligned}
& \left|\frac{\partial^{3} A^{\prime}[u]}{A^{\prime}[u+t g] A^{\prime}[u]^{2}}\right| \leq C b \frac{\sin ^{2}\left(\frac{\alpha^{\prime}}{2}\right)}{\left(\sin ^{2}\left(\frac{\alpha^{\prime}}{2}\right)+b^{2} s^{\prime 2}\right)^{3}}\left|\partial^{3}\left(u+u^{\prime}\right)\right|+C b^{2} \frac{\sin ^{2}\left(\frac{\alpha^{\prime}}{2}\right)}{\left(\sin ^{2}\left(\frac{\alpha^{\prime}}{2}\right)+b^{2} s^{\prime 2}\right)^{3}}\left|\partial u u^{\prime}\right| \\
& +C b^{2} \frac{\left|\sin \left(\frac{\alpha^{\prime}}{2}\right)\right|+\left|s^{\prime}\right|}{\left(\sin ^{2}\left(\frac{\alpha^{\prime}}{2}\right)+b^{2} s^{\prime 2}\right)^{2}}+C b^{2} \frac{\left|\sin \left(\frac{\alpha^{\prime}}{2}\right)\right|+\left|s^{\prime}\right|}{\left(\sin ^{2}\left(\frac{\alpha^{\prime}}{2}\right)+b^{2} s^{\prime 2}\right)^{3}}\left|\partial^{3}\left(u-u^{\prime}\right)\right| .
\end{aligned}
$$

and

$$
\begin{aligned}
& \left|\partial^{2} \frac{\partial A^{\prime}[u]}{A^{\prime}[u]^{2} A^{\prime}[u+t g]}-\frac{\partial^{3} A^{\prime}[u]}{A^{\prime}[u+t g] A^{\prime}[u]^{2}}\right| \leq C b^{2} \frac{\left(\sin ^{2}\left(\frac{\alpha^{\prime}}{2}\right)+b\left(\left|\sin \left(\frac{\alpha^{\prime}}{2}\right)\right|+\left|s^{\prime}\right|+s^{\prime 2}\right)\right)\left(\sin ^{2}\left(\frac{\alpha^{\prime}}{2}\right)+b s^{\prime 2}\right)}{\left(\sin ^{2}\left(\frac{\alpha^{\prime}}{2}\right)+b^{2} s^{\prime 2}\right)^{4}} \\
& +C b^{2} \frac{\left(\sin ^{2}\left(\frac{\alpha^{\prime}}{2}\right)+b\left(\left|\sin \left(\frac{\alpha^{\prime}}{2}\right)\right|+\left|s^{\prime}\right|+s^{\prime 2}\right)\right)\left(\sin ^{2}\left(\frac{\alpha^{\prime}}{2}\right)+b s^{\prime 2}\right)}{\left(\sin ^{2}\left(\frac{\alpha^{\prime}}{2}\right)+b^{2} s^{\prime 2}\right)^{3}}+C b^{3} \frac{\left(\sin ^{2}\left(\frac{\alpha^{\prime}}{2}\right)+b s^{\prime 2}\right)^{3}}{\left(\sin ^{2}\left(\frac{\alpha^{\prime}}{2}\right)+b^{2} s^{\prime 2}\right)^{4}} \\
& +C b^{3} \frac{\left(\sin ^{2}\left(\frac{\alpha^{\prime}}{2}\right)+b s^{\prime 2}\right)^{3}}{\left(\sin ^{2}\left(\frac{\alpha^{\prime}}{2}\right)+b^{2} s^{\prime 2}\right)^{5}}+C b^{2} \frac{\left(\sin ^{2}\left(\frac{\alpha^{\prime}}{2}\right)+b\left(\left|\sin \left(\frac{\alpha^{\prime}}{2}\right)\right|+\left|s^{\prime}\right|+s^{\prime 2}\right)\right)\left(\sin ^{2}\left(\frac{\alpha^{\prime}}{2}\right)+b s^{\prime 2}\right)}{\left(\sin ^{2}\left(\frac{\alpha^{\prime}}{2}\right)+b^{2} s^{\prime 2}\right)^{4}} \\
& +C b^{3} \frac{\left(\sin ^{2}\left(\frac{\alpha^{\prime}}{2}\right)+b s^{\prime 2}\right)\left(\sin ^{2}\left(\frac{\alpha^{\prime}}{2}\right)+b\left(\left|\sin \left(\frac{\alpha^{\prime}}{2}\right)\right|+\left|s^{\prime}\right|+s^{\prime 2}\right)\right)}{\left(\sin ^{2}\left(\frac{\alpha^{\prime}}{2}\right)+b^{2} s^{\prime 2}\right)^{5}} \\
& \leq C b^{2} \frac{\left(\sin ^{2}\left(\frac{\alpha^{\prime}}{2}\right)+b\left(\left|\sin \left(\frac{\alpha^{\prime}}{2}\right)\right|+\left|s^{\prime}\right|+s^{\prime 2}\right)\right)\left(\sin ^{2}\left(\frac{\alpha^{\prime}}{2}\right)+b s^{\prime 2}\right)}{\left(\sin ^{2}\left(\frac{\alpha^{\prime}}{2}\right)+b^{2} s^{\prime 2}\right)^{4}} \\
& +C b^{3} \frac{\left(\sin ^{2}\left(\frac{\alpha^{\prime}}{2}\right)+b s^{\prime 2}\right)^{3}}{\left(\sin ^{2}\left(\frac{\alpha^{\prime}}{2}\right)+b^{2} s^{\prime 2}\right)^{5}}+C b^{2} \frac{\left(\sin ^{2}\left(\frac{\alpha^{\prime}}{2}\right)+b\left(\left|\sin \left(\frac{\alpha^{\prime}}{2}\right)\right|+\left|s^{\prime}\right|+s^{\prime 2}\right)\right)\left(\sin ^{2}\left(\frac{\alpha^{\prime}}{2}\right)+b s^{\prime 2}\right)}{\left(\sin ^{2}\left(\frac{\alpha^{\prime}}{2}\right)+b^{2} s^{\prime 2}\right)^{4}} \\
& +C b^{2} \frac{\left(\sin ^{2}\left(\frac{\alpha^{\prime}}{2}\right)+b\left(\left|\sin \left(\frac{\alpha^{\prime}}{2}\right)\right|+\left|s^{\prime}\right|+s^{\prime 2}\right)\right)\left(\sin ^{2}\left(\frac{\alpha^{\prime}}{2}\right)+b s^{\prime 2}\right)}{\left(\sin ^{2}\left(\frac{\alpha^{\prime}}{2}\right)+b^{2} s^{\prime 2}\right)^{4}}
\end{aligned}
$$


And we can estimate

$$
\begin{aligned}
& \left|J_{311}\right| \leq C b^{3} t\left(\frac{\left(\sin ^{2}\left(\frac{\alpha^{\prime}}{2}\right)+b\left(\left|\sin \left(\frac{\alpha^{\prime}}{2}\right)\right|+s^{\prime 2}+\left|s^{\prime}\right|\right)\right)\left(\sin ^{2}\left(\frac{\alpha^{\prime}}{2}\right)+b s^{\prime 2}\right)^{2}}{\left(\sin ^{2}\left(\frac{\alpha^{\prime}}{2}\right)+b^{2} s^{\prime 2}\right)^{3}}\right) \\
& +C b^{4} t \frac{\left(\sin ^{2}\left(\frac{\alpha^{\prime}}{2}\right)+b s^{\prime 2}\right)^{4}}{\left(\sin ^{2}\left(\frac{\alpha^{\prime}}{2}\right)+b^{2} s^{\prime 2}\right)^{4}} \\
& \left|J_{312}\right| \leq C b^{2} t\left(\sin ^{2}\left(\frac{\alpha^{\prime}}{2}\right)+b s^{\prime 2}\right)^{2} \\
& \times\left(b \frac{\sin ^{2}\left(\frac{\alpha^{\prime}}{2}\right)}{\left(\sin ^{2}\left(\frac{\alpha^{\prime}}{2}\right)+b^{2} s^{\prime 2}\right)^{3}}\left|\partial^{3}\left(u+u^{\prime}\right)\right|+b^{2} \frac{\sin ^{2}\left(\frac{\alpha^{\prime}}{2}\right)}{\left(\sin ^{2}\left(\frac{\alpha^{\prime}}{2}\right)+b^{2} s^{\prime 2}\right)^{3}}\left|\partial^{3}\left(u u^{\prime}\right)\right|\right. \\
& +b^{2} \frac{\left|\sin \left(\frac{\alpha^{\prime}}{2}\right)\right|+\left|s^{\prime}\right|}{\left(\sin ^{2}\left(\frac{\alpha^{\prime}}{2}\right)+b^{2} s^{\prime 2}\right)^{2}}+C b^{2} \frac{\left|\sin \left(\frac{\alpha^{\prime}}{2}\right)\right|+\left|s^{\prime}\right|}{\left(\sin ^{2}\left(\frac{\alpha^{\prime}}{2}\right)+b^{2} s^{\prime 2}\right)^{3}}\left|\partial^{3}\left(u-u^{\prime}\right)\right| \\
& +b^{2} \frac{\left(\sin ^{2}\left(\frac{\alpha^{\prime}}{2}\right)+b\left(\left|\sin \left(\frac{\alpha^{\prime}}{2}\right)\right|+\left|s^{\prime}\right|+s^{\prime 2}\right)\right)\left(\sin ^{2}\left(\frac{\alpha^{\prime}}{2}\right)+b s^{\prime 2}\right)}{\left(\sin ^{2}\left(\frac{\alpha^{\prime}}{2}\right)+b^{2} s^{\prime 2}\right)^{4}} \\
& +b^{3} \frac{\left(\sin ^{2}\left(\frac{\alpha^{\prime}}{2}\right)+b s^{\prime 2}\right)^{3}}{\left(\sin ^{2}\left(\frac{\alpha^{\prime}}{2}\right)+b^{2} s^{\prime 2}\right)^{5}}+b^{2} \frac{\left(\sin ^{2}\left(\frac{\alpha^{\prime}}{2}\right)+b\left(\left|\sin \left(\frac{\alpha^{\prime}}{2}\right)\right|+\left|s^{\prime}\right|+s^{\prime 2}\right)\right)\left(\sin ^{2}\left(\frac{\alpha^{\prime}}{2}\right)+b s^{\prime 2}\right)}{\left(\sin ^{2}\left(\frac{\alpha^{\prime}}{2}\right)+b^{2} s^{\prime 2}\right)^{4}} \\
& \left.+b^{2} \frac{\left(\sin ^{2}\left(\frac{\alpha^{\prime}}{2}\right)+b\left(\left|\sin \left(\frac{\alpha^{\prime}}{2}\right)\right|+\left|s^{\prime}\right|+s^{\prime 2}\right)\right)\left(\sin ^{2}\left(\frac{\alpha^{\prime}}{2}\right)+b s^{\prime 2}\right)}{\left(\sin ^{2}\left(\frac{\alpha^{\prime}}{2}\right)+b^{2} s^{\prime 2}\right)^{4}}\right) .
\end{aligned}
$$

For $J_{32}$ we have that

$$
\begin{aligned}
& \partial J_{32}=\frac{2}{t} \partial\left(\frac{\partial A^{\prime}[u]}{A^{\prime}[u]^{2} A^{\prime}[u+t g]}\right)\left(A^{\prime}[u]-A^{\prime}[u+t g]\right)\left(\partial A^{\prime}[u]-\partial A^{\prime}[u+t g]\right) \\
& +\frac{2}{t} \frac{\partial A^{\prime}[u]}{A^{\prime}[u]^{2} A^{\prime}[u+t g]}\left(\partial A-\partial A^{\prime}[u+t g]\right)^{2} \\
& +\frac{2}{t} \frac{\partial A^{\prime}[u]}{A^{\prime}[u]^{2} A^{\prime}[u+t g]}\left(A^{\prime}[u]-A^{\prime}[u+t g]\right)\left(\partial^{2} A^{\prime}[u]-\partial^{2} A^{\prime}[u+t g]\right) \\
& \equiv J_{321}+J_{322}+J_{323}
\end{aligned}
$$

where $J_{321}=J_{311}$. Then we need to estimate using lemma B.1

$$
\begin{aligned}
& \left|J_{322}\right| \leq C b^{3} t \frac{\left(\sin ^{2}\left(\frac{\alpha^{\prime}}{2}\right)+b s^{\prime 2}\right)^{3}}{\left(\sin ^{2}\left(\frac{\alpha^{\prime}}{2}\right)+b^{2} s^{\prime 2}\right)^{3}} \\
& \left|J_{323}\right| \leq C b^{3} t \frac{\left(\sin ^{2}\left(\frac{\alpha^{\prime}}{2}\right)+b s^{\prime 2}\right)^{2}}{\left(\sin ^{2}\left(\frac{\alpha^{\prime}}{2}\right)+b^{2} s^{\prime 2}\right)^{3}}\left(\sin ^{2}\left(\frac{\alpha^{\prime}}{2}\right)+b\left(\left|\sin \left(\frac{\alpha^{\prime}}{2}\right)\right|+s^{\prime 2}+\left|s^{\prime}\right|\right)\right)
\end{aligned}
$$


Finally for $J_{42}$ we have that

$$
\begin{aligned}
& \partial J_{42}=\partial^{3}\left(\frac{1}{A}\right) \frac{1}{t}\left(A-A^{\prime}[u+t g]+t \partial_{u} A[g]\right)+\partial^{2}\left(\frac{1}{A^{\prime}[u]}\right) \frac{1}{t}\left(\partial A-\partial A g+t \partial_{u} A[g]\right) \\
& \equiv J_{421}+J_{422},
\end{aligned}
$$

where $J_{422}=J_{221}$. And then we just have to bound $J_{421}$. We notice that

$$
\partial^{3} \frac{1}{A^{\prime}[u]}=\frac{\partial^{3} A^{\prime}[u]}{A^{\prime}[u]^{2}}-6 \frac{\partial^{2} A^{\prime}[u] \partial A^{\prime}[u]}{A^{\prime}[u]^{3}}+6 \frac{\partial A^{\prime}[u]^{3}}{A^{\prime}[u]^{4}}
$$

And using lemma B.1 we have that

$$
\begin{aligned}
& \frac{\partial^{3} A^{\prime}[u]}{A^{\prime}[u]^{2}} \leq C b \frac{\sin ^{2}\left(\frac{\alpha^{\prime}}{2}\right)}{\left(\sin ^{2}\left(\frac{\alpha^{\prime}}{2}\right)+b^{2} s^{\prime 2}\right)^{2}}\left|\partial^{3}\left(u+u^{\prime}\right)\right|+C b^{2} \frac{\sin ^{2}\left(\frac{\alpha^{\prime}}{2}\right)}{\left(\sin ^{2}\left(\frac{\alpha^{\prime}}{2}\right)+b^{2} s^{\prime 2}\right)^{2}}\left|\partial u u^{\prime}\right| \\
& +C b^{2} \frac{\left|\sin \left(\frac{\alpha^{\prime}}{2}\right)\right|+\left|s^{\prime}\right|}{\left(\sin ^{2}\left(\frac{\alpha^{\prime}}{2}\right)+b^{2} s^{\prime 2}\right)^{2}}+C b^{2} \frac{\left|\sin \left(\frac{\alpha^{\prime}}{2}\right)\right|+\left|s^{\prime}\right|}{\left(\sin ^{2}\left(\frac{\alpha^{\prime}}{2}\right)+b^{2} s^{\prime 2}\right)^{2}}\left|\partial^{3}\left(u-u^{\prime}\right)\right| \\
& \left|\frac{\partial^{2} A^{\prime}[u] \partial A^{\prime}[u]}{A^{\prime}[u]^{3}}\right| \leq C b^{2} \frac{\left(\sin ^{2}\left(\frac{\alpha^{\prime}}{2}\right)+b\left(\left|\sin \left(\frac{\alpha^{\prime}}{2}\right)\right|+s^{\prime 2}+\left|s^{\prime}\right|\right)\left(\sin ^{2}\left(\frac{\alpha^{\prime}}{2}\right)+b s^{\prime 2}\right)\right)}{\left(\sin ^{2}\left(\frac{\alpha^{\prime}}{2}\right)+b^{2} s^{\prime 2}\right)^{3}} \\
& \left|\frac{\partial A^{\prime}[u]^{3}}{A^{\prime}[u]^{4}}\right| \leq C b^{3} \frac{\left(\sin ^{2}\left(\frac{\alpha^{\prime}}{2}\right)+b s^{\prime 2}\right)^{3}}{\left(\sin ^{2}\left(\frac{\alpha^{\prime}}{2}\right)+b^{2} s^{\prime 2}\right)^{4}} . \\
& \left|J_{422}\right| \leq C b^{2} t\left(\sin ^{2}\left(\frac{\alpha^{\prime}}{2}\right)+s^{\prime 2}\right) \\
& \times\left(b \frac{\sin ^{2}\left(\frac{\alpha^{\prime}}{2}\right)}{\left(\sin ^{2}\left(\frac{\alpha^{\prime}}{2}\right)+b^{2} s^{\prime 2}\right)^{2}}\left|\partial^{3}\left(u+u^{\prime}\right)\right|+b^{2} \frac{\sin ^{2}\left(\frac{\alpha^{\prime}}{2}\right)}{\left(\sin ^{2}\left(\frac{\alpha^{\prime}}{2}\right)+b^{2} s^{\prime 2}\right)^{2}}\left|\partial^{3}\left(u u^{\prime}\right)\right|\right. \\
& +b^{2} \frac{\left|\sin \left(\frac{\alpha^{\prime}}{2}\right)\right|+\left|s^{\prime}\right|}{\left(\sin ^{2}\left(\frac{\alpha^{\prime}}{2}\right)+b^{2} s^{\prime 2}\right)^{2}}+b^{2} \frac{\left|\sin \left(\frac{\alpha^{\prime}}{2}\right)\right|+\left|s^{\prime}\right|}{\left(\sin ^{2}\left(\frac{\alpha^{\prime}}{2}\right)+b^{2} s^{\prime 2}\right)^{2}}\left|\partial^{3}\left(u-u^{\prime}\right)\right| \\
& b^{2} \frac{\left(\sin ^{2}\left(\frac{\alpha^{\prime}}{2}\right)+b\left(\left|\sin \left(\frac{\alpha^{\prime}}{2}\right)\right|+s^{\prime 2}+\left|s^{\prime}\right|\right)\left(\sin ^{2}\left(\frac{\alpha^{\prime}}{2}\right)+b s^{\prime 2}\right)\right)}{\left(\sin ^{2}\left(\frac{\alpha^{\prime}}{2}\right)+b^{2} s^{\prime 2}\right)^{3}} \\
& \left.b^{3} \frac{\left(\sin ^{2}\left(\frac{\alpha^{\prime}}{2}\right)+b s^{\prime 2}\right)^{3}}{\left(\sin ^{2}\left(\frac{\alpha^{\prime}}{2}\right)+b^{2} s^{\prime 2}\right)^{4}}\right) \text {. }
\end{aligned}
$$

This finishes the proof of the lemma.

Lemma B.22 Let $g \in H^{4,3}$ with $\|g\|_{H^{4,3}}=1$ and $f(\alpha, s) \in L^{2}(\Omega)$ with $\operatorname{supp}(f) \subset \mathbb{T} \times[-1,1]$. Then the following estimates hold:

1.

$$
\left\|\int_{-\infty}^{\infty} \int_{-\pi}^{\pi} \partial^{j}\left(\frac{1}{t} \log \left(\frac{A^{\prime}[u+t g]}{A^{\prime}[u]}\right)-\frac{\partial_{u} A^{\prime}[g]}{A^{\prime}[u]}\right) f^{\prime} d \alpha^{\prime} d s^{\prime}\right\|_{L^{2}} \leq C b t
$$


2.

$$
\left\|\int_{-\infty}^{\infty} \int_{-\pi}^{\pi} \partial^{j}\left(\log \left(\frac{A^{\prime}[u+t g]}{A^{\prime}[u]}\right)\right) f^{\prime} d \alpha^{\prime} d s^{\prime}\right\|_{L^{2}} \leq C b t
$$

for $j=0,1,2$.

3.

$$
\left\|\int_{-\infty}^{\infty} \int_{-\pi}^{\pi}\left(\partial^{3}\left(\frac{1}{t} \log \left(\frac{A^{\prime}[u+t g]}{A^{\prime}[u]}\right)-\partial_{u} A^{\prime}[g]\right)-Z\left(\alpha, \alpha^{\prime}, s, s^{\prime}\right)\right) f\left(\alpha^{\prime}, s^{\prime}\right) d \alpha^{\prime} d s^{\prime}\right\|_{L^{2}} \leq C b t
$$

where

$$
\begin{aligned}
& Z\left(\alpha, \alpha^{\prime}, s, s^{\prime}\right)=\frac{1}{t} \frac{\left(\partial^{3} A^{\prime}[u+t g]-\partial^{3} A^{\prime}[u]\right)\left(A^{\prime}[u+t g]-A^{\prime}[u]\right)}{A^{\prime}[u+t g] A^{\prime}[u]}-\frac{1}{t} \frac{\partial^{3} A^{\prime}[u+t g]-\partial^{3} A^{\prime}[u]-t \partial^{3} \partial_{u} A^{\prime}[g]}{A^{\prime}[u]} \\
& +\frac{\partial^{3} A^{\prime}[u]}{t} \frac{\left(A^{\prime}[u]-A^{\prime}[u+t g]\right)^{2}}{A^{\prime}[u]^{2} A^{\prime}[u+t g]} .
\end{aligned}
$$

4.

$$
\left\|\int_{-\infty}^{\infty} \int_{-\pi}^{\pi}\left(\partial^{3} \log \left(\frac{A^{\prime}[u+t g]}{A^{\prime}[u]}\right)-W\left(\alpha, \alpha^{\prime}, s, s^{\prime}\right)\right) d \alpha^{\prime} d s^{\prime}\right\|_{L^{2}} \leq C b t
$$

where

$$
W\left(\alpha, \alpha^{\prime}, s, s^{\prime}\right)=\partial^{3} A^{\prime}[u]\left(\frac{1}{A^{\prime}[u+t g]}-\frac{1}{A^{\prime}[u]}\right)+\frac{\left(\partial^{3} A^{\prime}[u+t g]-\partial^{3} A^{\prime}[u]\right)}{A^{\prime}[u+t g]}
$$

Proof: The proof follows from Lemma B.21, Lemma B.2 and Minkowski's inequality.

Lemma B.23 Let $g \in H^{4,3}$ with $\|g\|_{H^{4,3}}=1$ and $f(\alpha, s) \in L^{\infty}(\Omega)$ with $\operatorname{supp}(f) \subset \mathbb{T} \times[-1,1]$. Then the following estimates hold:

$$
\left\|\int_{-\infty}^{\infty} \int_{-\pi}^{\pi} Z\left(\alpha, \alpha^{\prime}, s, s^{\prime}\right) f^{\prime} d \alpha^{\prime} d s^{\prime}\right\|_{L^{2}} \leq C b t
$$

where

$$
\begin{gathered}
Z\left(\alpha, \alpha^{\prime}, s, s^{\prime}\right)=\frac{1}{t} \frac{\left(\partial^{3} A^{\prime}[u+t g]-\partial^{3} A^{\prime}[u]\right)\left(A^{\prime}[u+t g]-A^{\prime}[u]\right)}{A^{\prime}[u+t g] A^{\prime}[u]}-\frac{1}{t} \frac{\partial^{3} A^{\prime}[u+t g]-\partial^{3} A^{\prime}[u]-t \partial^{3} \partial_{u} A^{\prime}[g]}{A^{\prime}[u]} \\
+\frac{\partial^{3} A^{\prime}[u]}{t} \frac{\left(A^{\prime}[u]-A^{\prime}[u+t g]\right)^{2}}{A^{\prime}[u]^{2} A^{\prime}[u+t g]} . \\
\left\|\int_{-\infty}^{\infty} \int_{-\pi}^{\pi} W\left(\alpha, \alpha^{\prime}, s, s^{\prime}\right) f\left(\alpha^{\prime}, s^{\prime}\right) d \alpha^{\prime} d s^{\prime}\right\|_{L^{2}} \leq C b t
\end{gathered}
$$

where

$$
W\left(\alpha, \alpha^{\prime}, s, s^{\prime}\right)=\partial^{3} A^{\prime}[u]\left(\frac{1}{A^{\prime}[u+t g]}-\frac{1}{A^{\prime}[u]}\right)+\frac{\left(\partial^{3} A^{\prime}[u+t g]-\partial^{3} A^{\prime}[u]\right)}{A^{\prime}[u+t g]} .
$$


Proof: The proof follows from Lemma B.21, Lemma B.2 and Minkowski's inequality.

Lemma B.24 Let $g \in H^{4,3}$ with $\|g\|_{H^{4,3}}=1$ and $f(\alpha, s) \in L^{2}(\Omega)$ with supp $(f) \subset \mathbb{T} \times[-1,1]$. Then the following estimates hold:

$$
\left\|\int_{-\infty}^{\infty} \int_{-\pi}^{\pi} \partial^{j} \frac{1}{b}\left(\frac{1}{t} \log \left(\frac{A^{\prime}[u+t g]}{A^{\prime}[u]}\right)-\frac{\partial_{u} A^{\prime}[g]}{A^{\prime}[u]}\right)\left|\sin \left(\frac{\alpha^{\prime}}{2}\right)\right| f^{\prime} d \alpha^{\prime} d s^{\prime}\right\|_{L^{2}} \leq C b t \log \left(\frac{1}{b}\right)
$$

for $j=0,1,2$.

$\left\|\int_{-\infty}^{\infty} \int_{-\pi}^{\pi} \frac{1}{b}\left(\partial^{3}\left(\frac{1}{t} \log \left(\frac{A^{\prime}[u+t g]}{A^{\prime}[u]}\right)-\partial_{u} A^{\prime}[g]\right)-Z\left(\alpha, \alpha^{\prime}, s, s^{\prime}\right)\right)\left|\sin \left(\frac{\alpha^{\prime}}{2}\right)\right| f\left(\alpha^{\prime}, s^{\prime}\right) d \alpha^{\prime} d s^{\prime}\right\|_{L^{2}} \leq C b t \log \left(\frac{1}{b}\right)$

where

$Z\left(\alpha, \alpha^{\prime}, s, s^{\prime}\right)=\frac{1}{t} \frac{\left(\partial^{3} A^{\prime}[u+t g]-\partial^{3} A^{\prime}[u]\right)\left(A^{\prime}[u+t g]-A^{\prime}[u]\right)}{A^{\prime}[u+t g] A^{\prime}[u]}-\frac{1}{t} \frac{\partial^{3} A^{\prime}[u+t g]-\partial^{3} A^{\prime}[u]-t \partial^{3} \partial_{u} A^{\prime}[g]}{A^{\prime}[u]}$

$+\frac{\partial^{3} A^{\prime}[u]}{t} \frac{\left(A^{\prime}[u]-A^{\prime}[u+t g]\right)^{2}}{A^{\prime}[u]^{2} A^{\prime}[u+t g]}$.

Proof: The proof is as in lemma B.22 but using the extra $\sin \left(\frac{\alpha^{\prime}}{2}\right)$ of this term.

Lemma B.25 Let $g \in H^{4,3}$ with $\|g\|_{H^{4,3}}=1$ and $f(\alpha, s) \in L^{\infty}(\Omega)$ with $\operatorname{supp}(f) \subset \mathbb{T} \times[-1,1]$. Then the following estimates hold:

$$
\left\|\int_{-\infty}^{\infty} \int_{-\pi}^{\pi} Z\left(\alpha, \alpha^{\prime}, s, s^{\prime}\right)\left|\sin \left(\frac{\alpha^{\prime}}{2}\right)\right| f^{\prime} d \alpha^{\prime} d s^{\prime}\right\|_{L^{2}} \leq C b t \log \left(\frac{1}{b}\right)
$$

where

$Z\left(\alpha, \alpha^{\prime}, s, s^{\prime}\right)=\frac{1}{t} \frac{\left(\partial^{3} A^{\prime}[u+t g]-\partial^{3} A^{\prime}[u]\right)\left(A^{\prime}[u+t g]-A^{\prime}[u]\right)}{A^{\prime}[u+t g] A^{\prime}[u]}-\frac{1}{t} \frac{\partial^{3} A^{\prime}[u+t g]-\partial^{3} A^{\prime}[u]-t \partial^{3} \partial_{u} A^{\prime}[g]}{A^{\prime}[u]}$

$+\frac{\partial^{3} A^{\prime}[u]}{t} \frac{\left(A^{\prime}[u]-A^{\prime}[u+t g]\right)^{2}}{A^{\prime}[u]^{2} A^{\prime}[u+t g]}$.

Proof: The proof is as in lemma B.23 but using the extra $\sin \left(\frac{\alpha^{\prime}}{2}\right)$ of this term.

Lemma B.26 The following estimate holds

$$
\left\|\int_{-\infty}^{\infty} \int_{-\pi}^{\pi} F_{s}\left(s-s^{\prime}\right)\left(-\frac{2}{a^{3}} \frac{1}{t} \log \left(\frac{A^{\prime}[u+t g]}{A^{\prime}[u]}\right)+\frac{2}{a^{3}} \frac{\partial_{u} A^{\prime}[u]}{A^{\prime}[u]}\right)\left|\sin \left(\alpha^{\prime}\right)\right| d \alpha^{\prime} d s^{\prime}\right\|_{H^{3}} \leq C a \log \left(\frac{1}{b}\right)
$$

Proof: The proof is similar to that one in lemmas B.24 and B.25 but you have to divide between $a$, because we are taking a derivative in $a$.

Lemma B.27 The following estimates hold

$$
\left\|\int_{-\infty}^{\infty} \int_{-\pi}^{\pi} F_{\rho}\left(s-s^{\prime}\right) \frac{1}{a^{2}}\left(\frac{1}{t} \frac{\partial_{a} A^{\prime}[u+t g]-\partial_{a} A^{\prime}[u]-t \partial_{u} \partial_{a} A[g]}{A^{\prime}[u+t g]}\right)\left|\sin \left(\alpha^{\prime}\right)\right| d \alpha d s^{\prime}\right\|
$$




$$
\begin{aligned}
& +\left\|\int_{-\infty}^{\infty} \int_{-\pi}^{\pi} F_{\rho}\left(s-s^{\prime}\right) \frac{1}{a^{2}} \frac{\partial_{u} \partial_{a} A^{\prime}[g]}{A^{\prime}[u+t g] A^{\prime}[u]}\left(A^{\prime}[u]-A^{\prime}[u+t g]\right)\left|\sin \left(\alpha^{\prime}\right)\right| d \alpha^{\prime} d s^{\prime}\right\| \leq C a \log \left(\frac{1}{b}\right) \\
& \left\|\int_{-\infty}^{\infty} \int_{-\pi}^{\pi} F_{\rho}\left(s-s^{\prime}\right) \frac{1}{a^{2}} \frac{\partial_{a} A^{\prime}[g]}{t A^{\prime}[u] A^{\prime}[u+t g]}\left(A^{\prime}[u]-A^{\prime}[u+t g]+t \partial_{u} A^{\prime}[g]\right)\left|\sin \left(\alpha^{\prime}\right)\right| d \alpha^{\prime} d s^{\prime}\right\| \\
& +\left\|\int_{-\infty}^{\infty} \int_{-\pi}^{\pi} F_{\rho}\left(s-s^{\prime}\right) \frac{1}{a^{2}} \frac{\partial_{a} A[g] \partial_{u} A[g]}{A^{\prime}[u+t g] A^{\prime}[u]^{2}}\left(A^{\prime}[u+t g]-A^{\prime}[u]\right)\left|\sin \left(\alpha^{\prime}\right)\right| d \alpha^{\prime} d s^{\prime}\right\| \leq C a \log \left(\frac{1}{b}\right) .
\end{aligned}
$$

Proof: The proof is similar to that one of lemmas B.24 and B.25 but dividing by $a$ because we lose a derivative in $a$.

Lemma B.28 The following estimates hold

$$
\begin{aligned}
& \left\|\int_{-\infty}^{\infty} \int_{-\pi}^{\pi} F_{s}\left(s-s^{\prime}\right) \frac{1}{t}\left(\frac{\partial_{a} A^{\prime}[u+t g]-\partial_{a} A^{\prime}[u]-t \partial_{u} \partial_{a} A^{\prime}[u]}{A[u+t g]}\right) \cos \left(\alpha^{\prime}\right) u_{\alpha}^{\prime} d \alpha^{\prime} d s^{\prime}\right\|_{H^{3}} \\
& +\left\|\int_{-\infty}^{\infty} \int_{-\pi}^{\pi} F_{s}\left(s-s^{\prime}\right) \partial_{u} \partial_{a} A^{\prime}[u+t g]\left(\frac{1}{A^{\prime}[u+t g]}-\frac{1}{A^{\prime}[u]}\right) \cos \left(\alpha^{\prime}\right) u_{\alpha}^{\prime} d \alpha^{\prime} d s^{\prime}\right\|_{H^{3}} \leq C a \log \left(\frac{1}{b}\right) \\
& \left\|\int_{-\infty}^{\infty} \int_{-\pi}^{\pi} f_{s}\left(s-s^{\prime}\right) \partial_{a} A^{\prime}[u] \frac{1}{t} \frac{A^{\prime}[u]-A^{\prime}[u+t g]+t \partial_{u} A^{\prime}[u]}{A^{\prime}[u+t g] A^{\prime}[u]} \cos \left(\alpha^{\prime}\right) u_{\alpha}^{\prime} d \alpha^{\prime} d s^{\prime}\right\| \\
& +\left\|\int_{-\infty}^{\infty} \int_{-\pi}^{\pi} F_{s}\left(s-s^{\prime}\right) \frac{\partial_{a} A^{\prime}[u] \partial_{u} A^{\prime}[u]}{A^{\prime}[u]}\left(\frac{1}{A^{\prime}[u+t g]}-\frac{1}{A^{\prime}[u]}\right) \cos \left(\alpha^{\prime}\right) u_{\alpha}^{\prime} d \alpha^{\prime} d s^{\prime}\right\| \leq C a \log \left(\frac{1}{b}\right) .
\end{aligned}
$$

Proof: The proof of this lemma is similar to the proofs of lemmas B.22 and B.23 but dividing by $a$ since we are taking a derivative in this parameter.

Conflict of Interest: The authors declare that they have no conflict of interest.

\section{References}

[1] V. Arnol'd. Sur la géométrie différentielle des groupes de Lie de dimension infinie et ses applications à l'hydrodynamique des fluides parfaits. Ann. Inst. Fourier (Grenoble), 16(fasc. 1):319-361, 1966.

[2] V. I. Arnol'd. An a priori estimate in the theory of hydrodynamic stability. Izv. Vyš̌. Učebn. Zaved. Matematika, 1966(5 (54)):3-5, 1966.

[3] V. I. Arnold and B. A. Khesin. Topological methods in hydrodynamics, volume 125 of Applied Mathematical Sciences. Springer-Verlag, New York, 1998.

[4] J. Bedrossian and N. Masmoudi. Inviscid damping and the asymptotic stability of planar shear flows in the 2D Euler equations. Publ. Math. Inst. Hautes Études Sci., 122:195-300, 2015.

[5] A. L. Bertozzi and P. Constantin. Global regularity for vortex patches. Comm. Math. Phys., 152(1):19-28, 1993.

[6] J. Burbea. Motions of vortex patches. Lett. Math. Phys., 6(1):1-16, 1982.

[7] A. Castro, D. Córdoba, and J. Gómez-Serrano. Existence and regularity of rotating global solutions for the generalized surface quasi-geostrophic equations. Duke Math. J., 165(5):935-984, 2016. 
[8] A. Castro, D. Córdoba, and J. Gómez-Serrano. Global smooth solutions for the inviscid SQG equation. Arxiv preprint arXiv:1603.03325, 2016.

[9] A. Castro, D. Córdoba, and J. Gómez-Serrano. Uniformly rotating analytic global patch solutions for active scalars. Annals of PDE, 2(1):1-34, 2016.

[10] J.-Y. Chemin. Persistance de structures géométriques dans les fluides incompressibles bidimensionnels. Ann. Sci. École Norm. Sup. (4), 26(4):517-542, 1993.

[11] A. Choffrut and V. Šverák. Local structure of the set of steady-state solutions to the 2D incompressible Euler equations. Geom. Funct. Anal., 22(1):136-201, 2012.

[12] A. Choffrut and L. Székelyhidi, Jr. Weak solutions to the stationary incompressible Euler equations. SIAM J. Math. Anal., 46(6):4060-4074, 2014.

[13] M. G. Crandall and P. H. Rabinowitz. Bifurcation from simple eigenvalues. J. Functional Analysis, 8:321-340, 1971.

[14] F. de la Hoz, T. Hmidi, J. Mateu, and J. Verdera. Doubly connected $V$-states for the planar Euler equations. SIAM J. Math. Anal., 48(3):1892-1928, 2016.

[15] G. S. Deem and N. J. Zabusky. Vortex waves: Stationary "V-states", interactions, recurrence, and breaking. Physical Review Letters, 40(13):859-862, 1978.

[16] T. M. Elgindi and I.-J. Jeong. Symmetries and Critical Phenomena in Fluids. arXiv preprint arXiv:1610.09701, 2016.

[17] Z. Hassainia and T. Hmidi. On the V-states for the generalized quasi-geostrophic equations. Comm. Math. Phys., 337(1):321-377, 2015.

[18] T. Hmidi and J. Mateu. Existence of corotating and counter-rotating vortex pairs for active scalar equations. Communications in Mathematical Physics, pages 1-49, 2016.

[19] T. Hmidi, J. Mateu, and J. Verdera. Boundary regularity of rotating vortex patches. Archive for Rational Mechanics and Analysis, 209(1):171-208, 2013.

[20] A. Kiselev and V. Šverák. Small scale creation for solutions of the incompressible two-dimensional Euler equation. Ann. of Math. (2), 180(3):1205-1220, 2014.

[21] G. Luo and T. Y. Hou. Potentially singular solutions of the 3d axisymmetric euler equations. Proceedings of the National Academy of Sciences, 111(36):12968-12973, 2014.

[22] X. Luo and R. Shvydkoy. 2D homogeneous solutions to the Euler equation. Comm. Partial Differential Equations, 40(9):1666-1687, 2015.

[23] X. Luo and R. Shvydkoy. Addendum: 2D homogeneous solutions to the Euler equation. arXiv preprint arXiv:1608.00061, 2016.

[24] C. Marchioro and M. Pulvirenti. Mathematical theory of incompressible nonviscous fluids, volume 96 of Applied Mathematical Sciences. Springer-Verlag, New York, 1994.

[25] N. Nadirashvili. On stationary solutions of two-dimensional Euler equation. Arch. Ration. Mech. Anal., 209(3):729-745, 2013.

[26] P. Serfati. Une preuve directe d'existence globale des vortex patches 2D. C. R. Acad. Sci. Paris Sér. I Math., 318(6):515-518, 1994. 
[27] V. I. Yudovich. Non-stationary flows of an ideal incompressible fluid. Z̆. Vyčisl. Mat. i Mat. Fiz., 3:1032-1066, 1963.

\section{Angel Castro}

Departamento de Matemáticas

Universidad Autónoma de Madrid

Instituto de Ciencias Matemáticas-CSIC

C/ Nicolas Cabrera, 13-15, 28049 Madrid, Spain

Email: angel_castro@icmat.es

\section{Diego Córdoba}

Instituto de Ciencias Matemáticas

Consejo Superior de Investigaciones Científicas

C/ Nicolas Cabrera, 13-15, 28049 Madrid, Spain

Email: dcg@icmat.es

Javier Gómez-Serrano

Department of Mathematics

Princeton University

610 Fine Hall, Washington Rd,

Princeton, NJ 08544, USA

Email: jg27@math.princeton.edu 